\title{
Stable H-minimal hypersurfaces
}

\author{
Francescopaolo Montefalcone用
}

\begin{abstract}
We prove some stability results for smooth $H$-minimal hypersurfaces immersed in a sub-Riemannian $k$-step Carnot group $\mathbb{G}$. The main tools are the formulae for the 1 st and 2 nd variation of the $H$-perimeter measure $\sigma_{H}^{n-1}$. KEY WORDS AND PHRASES: Carnot groups; $H$-minimal hypersurfaces; 1 st and 2nd variation of the $H$-perimeter; stability; characteristic set. Mathematics SubJect Classification: 49Q15, 46E35, 22E60.
\end{abstract}

\section{Contents}

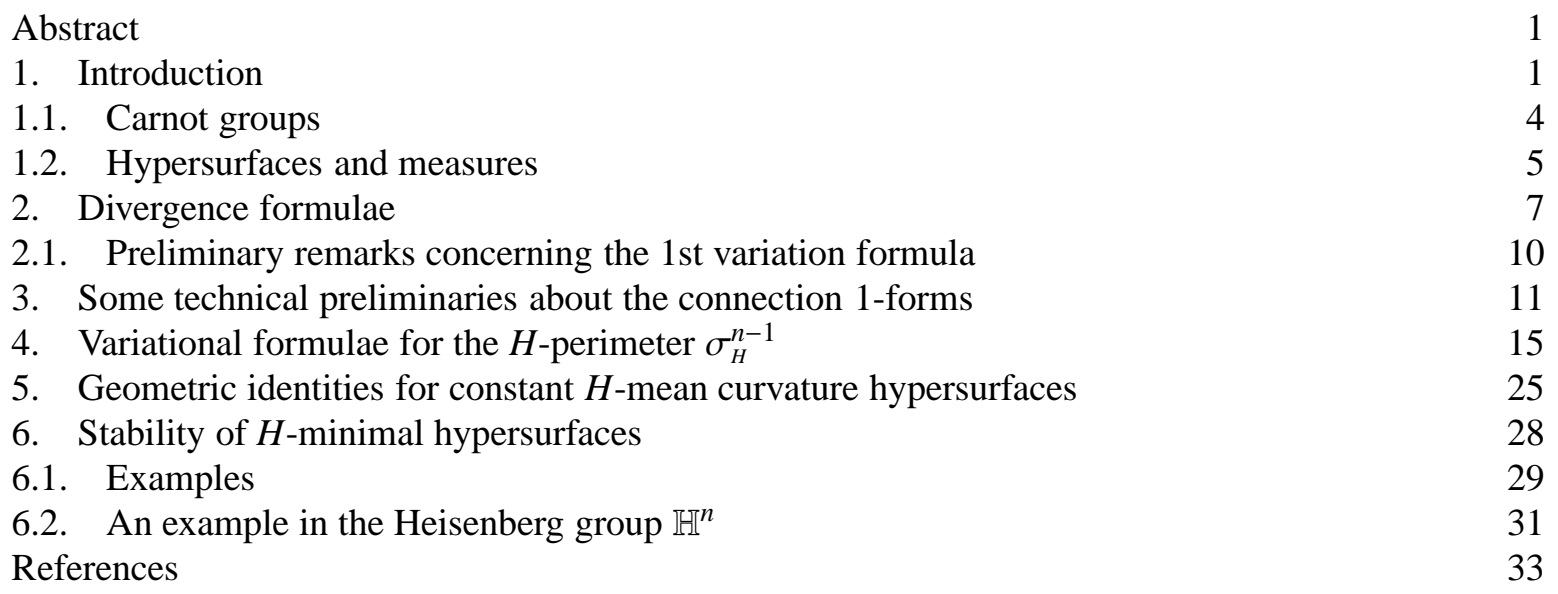

\section{INTRODUCTION}

In 3 Differential Geometry a minimal (hyper)surface of $\mathbb{R}^{n}$ (or, of a Riemannian manifold $\left(M^{n},\langle\cdot, \cdot\rangle\right)$ ) is a smooth codimension one submanifold having zero mean curvature. We recall that the Riemannian mean curvature $\mathcal{H}_{\mathcal{R}}$ of a hypersurface $S$ is the trace of its 2 nd fundamental form $B_{\mathcal{R}}$, which is the $\mathbf{C}^{\infty}$ bilinear form defined as $B_{\mathcal{R}}(X, Y):=\left\langle\nabla_{X} Y, v\right\rangle$ for every $X, Y \in \mathfrak{X}(T S):=\mathbf{C}^{\infty}(S, T S)$, where $\nabla$ denotes the Levi-Civita connection on the ambient space (either $\mathbb{R}^{n}$ or $M$ ) and $v$ is the unit normal vector along $S$. Note that $\mathcal{H}_{\mathcal{R}}=-\operatorname{div}_{T S} v$. Minimal hypersurfaces turn out to be critical points of the Riemannian $(n-1)$-dimensional volume $\sigma_{\mathcal{R}}^{n-1}$ and hence, studying stability of a minimal hypersurface $S$ means to study conditions under which $S$ turns out to be a minimum of the functional $\sigma_{\mathcal{R}}^{n-1}$. For this reason the 2nd variation formula of $\sigma_{\mathcal{R}}^{n-1}$ becomes a fundamental tool and, in order to avoid boundary contributions, we can use compactly supported normal variations of $S$. For an introduction to these topics in the Euclidean and/or Riemannian setting we refer the reader to the surveys by Chern [21], Lawson [47] and Osserman [61]; see also Simons' paper [71]. Finally, for some results concerning stability of minimal and CMC hypersurfaces, we would like to mention the papers [10], [11], [28], [31].

That of Minimal Surfaces is one of the great chapters of the XX century Mathematics, above all, because was a rich source of entirely new ideas and theories such as that of Currents, introduced by

\footnotetext{
${ }^{1}$ F. M. has been partially supported by the Fondazione CaRiPaRo Project "Nonlinear Partial Differential Equations: models, analysis, and control-theoretic problems".

${ }^{2}$ The author wishes to thank the anonymous referee for many helpful comments that improved the paper.

${ }^{3}$ Warning: In this new version, I have corrected some imprecisions and, more importantly, I have removed the last section of the previous version. The reason for this change have been some non-trivial modifications to the preprint [57]. No other substantial changes have been made.
} 
Federer and Fleming [30] (see Federer's fundamental treatise [29]), that of Sets of Finite Perimeter created by De Giorgi and its school starting from the pioneering work of Caccioppoli (see the book by Giusti [38] or [3]), and that of Varifolds, heavily inspired by Almgreen and developed by Allard in [1, 2]. A highly recommended introduction for these topics is, of course, the book by Simon [70]; see also the survey by Bombieri [14] and Morgan's book [60].

In this paper, we study some of these problems, in the sub-Riemannian setting of Carnot groups. We recall that a sub-Riemannian manifold is a smooth $n$-dimensional manifold $M$, endowed with a nonintegrable distribution $H \subset T M$ of $h$-planes, called the horizontal bundle, on which a (positive definite) metric $g_{H}$ is given. The horizontal bundle $H$ satisfies the Hörmander condition and this implies the validity of Chow theorem so that, different points can always be joined by horizontal curves (i.e. curves that are everywhere tangent to $H$ ). The idea is simply that, in connecting two points, we are only allowed to follow horizontal paths joining them. The $C C$-distance $d_{H}$, is then defined by minimizing the $g_{H}$ length of horizontal curves connecting two given points: this is the distance used in sub-Riemannian geometry. As an introduction to these topics, we refer the reader to Gromov [40], Montgomery [58], Pansu [62, 63], Strichartz [74]. In this context, Carnot groups play a role similar to Euclidean spaces in Riemannian geometry. They serve as a model for the tangent space of a sub-Riemannian manifold and, further, represent a wide class of examples of these geometries. By definition, a k-step Carnot group $\mathbb{G}$ is a $n$-dimensional, connected, simply connected and nilpotent Lie group (with respect to a group law $\bullet$ which is polynomial) having a $k$-step stratified Lie algebra $\mathfrak{g} \cong \mathbb{R}^{n}$. This means that $\mathfrak{g}$ splits into a direct sum of vector subspaces satisfying suitable commuting relations. More precisely, we have $\mathfrak{g}=H_{1} \oplus H_{2} \oplus \cdots \oplus H_{k},\left[H_{1}, H_{i}\right]=H_{i+1}$ for every $i=1, \ldots, k-1$ and $\left[H_{1}, H_{i}\right]=0$ for every $i \geq k$, where the brackets $[\cdot, \cdot]$ denote Lie brackets. We assume that $h_{i}=\operatorname{dim} H_{i}(i=1, \ldots, k)$ so that $n=\sum_{i=1}^{k} h_{i}$. The stratification of $\mathfrak{g}$ can be seen as the algebraic counterpart of the Hörmander condition.

We recall that Carnot groups are homogeneous groups, in the sense that they admit a family of positive anisotropic dilations modeled on the stratification; see [73]. This richness of geometric structures, makes interesting the study of Geometric Measure Theory in Carnot groups; see, for instance, [4], [5], [6], [9], [22], [36], [32, 33, 34, 35], [54, 55, 56], [50, 51, 52], [59] and bibliographies therein. We also cite [13], [17, 18], [19], [25, 26], [37], [64], [44], [66], [67] for many important results concerning $H$-minimal and/or constant horizontal mean curvature (hyper)surfaces of the Heisenberg group. Nevertheless, here we have to remark that not much is known about the geometry of smooth $H$-minimal hypersurfaces in general groups.

The aim of this paper, which is somehow a continuation of [56], is studying the stability of smooth $H$-minimal hypersurfaces immersed in $k$-step Carnot groups. Let us briefly describe our results.

In Section 1.1, we fix notation and main definitions concerning Carnot groups. We use a left invariant frame $\underline{X}:=\left\{X_{1}, \ldots, X_{n}\right\}$ on $\mathfrak{g}$ adapted to the stratification and fix a Riemannian metric $\langle\cdot, \cdot\rangle$ making $\underline{X}$ orthonormal (henceforth abbreviated as o.n.). This frame satisfies some non-trivial commuting relations encoded by the so-called structural constants $C_{i, j}^{\mathfrak{g} r}:=\left\langle\left[X_{i}, X_{j}\right], X_{r}\right\rangle \forall i, j, r=1, \ldots, n$. Note also that the (uniquely determined) left invariant Levi Civita connection $\nabla$ can be expressed in terms of structural constants. The projection of $\nabla$ onto the horizontal space $H$ is denoted by $\nabla^{H}$ and called horizontal connection.

In Section 1.2 we recall basic facts about immersed hypersurfaces endowed with the $H$-perimeter measure $\sigma_{H}^{n-1}$. Note that $\sigma_{H}^{n-1}=\left|\mathcal{P}_{H} v\right| \sigma_{\mathcal{R}}^{n-1}$, where $\sigma_{\mathcal{R}}^{n-1}$ is the $(n-1)$-dimensional Riemannian measure, $v$ is the unit (Riemannian) normal along $S$ and $\mathcal{P}_{H}$ is the projection onto $H$. Let $v_{H}=\frac{\mathcal{P}_{H} v}{\left|\mathcal{P}_{H} v\right|}$ be the unit horizontal normal along $S$ and let $H S \subset T S$ be the horizontal tangent space, which is $(h-1)$-dimensional at each non-characteristic point $p \in S \backslash C_{S}$, where $C_{S}:=\left\{p \in S:\left|\mathcal{P}_{H} v\right|=0\right\}$ denotes the characteristic set. It turns out that $H_{p}=H_{p} S \oplus \operatorname{span} \operatorname{R}_{\mathbb{R}}\left\{v_{H}(p)\right\}$ at each $p \in S \backslash C_{S}$. This allows us to define the horizontal 2nd fundamental form by setting $B_{H}(X, Y):=\left\langle\nabla_{X}^{H} Y, v_{H}\right\rangle$ for every $X, Y \in \mathbf{C}^{1}(S, H S)$. However, this object is not symmetric, in general. Thus it can be decomposed in its symmetric and skew-symmetric parts, i.e. $B_{H}=S_{H}+A_{H}$. 
In Section 2 we discuss some divergence-type formulae, which are very important tools. In particular, these results enable us to define the horizontal tangential operators $\mathcal{D}_{H S}$ and $\mathcal{L}_{H S}$, which are analogous, in this SR setting, to tangential divergence $d i v_{T S}$ and Laplacian $\Delta_{T S}$. An important fact is the validity of the formula

$$
-\int_{S} \varphi \mathcal{L}_{H S} \varphi \sigma_{H}^{n-1}=\int_{S}\left|\operatorname{grad}_{H S} \varphi\right|^{2} \sigma_{H}^{n-1}
$$

for every compactly supported function $\varphi \in \mathbf{C}_{H S}^{2}\left(S \backslash C_{S}\right) \cap W_{H S}^{1,2}\left(S ; \sigma_{H}^{n-1}\right)$; see Corollary 2.8 and also Remark 2.9. This formula holds (a fortiori) whenever $\varphi \in \mathbf{C}^{2}(S)$.

In Section 2.1 we discuss the basic calculations needed to prove the 1st variation formula for the $H$-perimeter $\sigma_{H}^{n-1}$.

Section 3 contains some other tools: adapted frames, connection 1-forms and lemmata concerning the horizontal 2nd fundamental form $B_{H}$. This material is then used in Section 4 to discuss and prove the variational formulae for $\sigma_{H}^{n-1}$. The presentation here is slightly different from [56]. In fact, we have tried to simplify the original proofs. More importantly, we have corrected a mistake that has caused the loss of some divergence-type terms in the variational formulae proved there; see Remark 2.14 Furthermore, we have extended the formulae to the characteristic case.

We say that a hypersurface $S$ of class $\mathbf{C}^{2}$ is $H$-minimal if its horizontal mean curvature $\mathcal{H}_{H}$ is zero at each non-characteristic $p \in S \backslash C_{S}$. Moreover, it turns out that the "infinitesimal"1st variation of $\sigma_{H}^{n-1}$ is given by

$$
\mathcal{L}_{W} \sigma_{H}^{n-1}=\left(-\mathcal{H}_{H}\langle W, v\rangle+\operatorname{div}_{T S}\left(W^{\top}\left|\mathcal{P}_{H} v\right|-\langle W, v\rangle v_{H}^{\top}\right)\right) \sigma_{\mathcal{R}}^{n-1},
$$

where $\mathcal{L}_{W} \sigma_{H}^{n-1}$ is the Lie derivative of $\sigma_{H}^{n-1}$ with respect to the initial velocity $W$ of the variation and the symbols $W^{\perp}, W^{\top}$ denote the normal and tangential components of $W$, respectively. If $\mathcal{H}_{H}$ is $L^{1}\left(S ; \sigma_{\mathcal{R}}^{n-1}\right)$, the function $\mathcal{L}_{W} \sigma_{H}^{n-1}$ is integrable on $S$ and the integral of $\mathcal{L}_{W} \sigma_{H}^{n-1}$ on $S$ gives the 1 st variation of $\sigma_{H}^{n-1}$. Note that the third term in the previous formula depends on the normal component of $W$. We stress that this term was omitted in [56]. Using a generalized divergence-type formula, the divergence term can be integrated on the boundary and, if one use compactly supported variations, it follows that $H$-minimal hypersurfaces are "critical points" of the $H$-perimeter functional.

The formula for 2nd variation of $\sigma_{H}^{n-1}$, which is one of the main results of this paper, will be obtained as a result of a long calculation; see Theorem 4.12. This formula will be proved under some further assumptions concerning integrability of some geometric quantities. For a precise statement, we refer the reader to Section 4 In the Heisenberg group $\mathbb{H}^{1}$, the 1st variation formula for characteristic surfaces of class $\mathbf{C}^{2}$ was first obtained by Ritoré and Rosales in [67]. We also stress that Hurtado, Ritoré and Rosales [44] have proved a formula for the 2 nd variation which is analogous to that stated in Theorem 4.12] We also quote [43], for similar results in a general sub-Riemannian setting.

Using compactly supported variations together with suitable integrability conditions on the function $\frac{1}{\left|\mathcal{P}_{H} v\right|}$, the 2nd variation formula takes the following simple form

$$
I_{S}\left(W, \sigma_{H}^{n-1}\right)=\int_{S}\left(\left|\operatorname{grad}_{H S} w\right|^{2}-w^{2} \mathcal{B}_{T S}\right) \sigma_{H}^{n-1},
$$

where $W$ is the variation vector and $w=\frac{\langle W, v\rangle}{\left|\mathcal{P}_{H} v\right|}$. Here, we have set

$$
\mathcal{B}_{T S}:=\underbrace{\left\|S_{H}\right\|_{\mathrm{G} r}^{2}+\left\|A_{H}\right\|_{\mathrm{G} r}^{2}}_{=\left\|B_{H}\right\|_{\mathrm{G} r}^{2}}+\sum_{\alpha \in I_{V}}\left\langle\left[2 \operatorname{grad}_{H S}\left(\varpi_{\alpha}\right)-C(\varpi)\left(X_{\alpha}-\varpi_{\alpha} v_{H}\right)\right], C^{\alpha} v_{H}\right\rangle ;
$$

see Corollary 4.13, Definition 1.7 and Definition 1.9 in Section 1.2 . Note that the above expression involves many geometric quantities such as the horizontal 2 nd fundamental form $B_{H}$ (or, its symmetric and skew-symmetric parts $S_{H}$ and $\left.A_{H}\right)$, the vertical vector field $\varpi$, defined as $\varpi:=\frac{\mathcal{P}_{V} v}{\left|\mathcal{P}_{H} v\right|}=\sum_{\alpha=h+1}^{n} \varpi_{\alpha} X_{\alpha}$, where $\varpi_{\alpha}:=\frac{v_{\alpha}}{\left|\mathcal{P}_{H} v\right|}$, and the matrices of the structural constants $C^{\alpha}$ and $C(\varpi)=\sum_{\alpha \in I_{V}} \varpi_{\alpha} C^{\alpha}$. 
In Section 5 we state some further identities for constant horizontal mean curvature hypersurfaces. In particular, we find a family of explicit solutions to the equation

$$
\mathcal{L}_{H S} \varphi+\varphi \mathcal{B}_{T S}=0
$$

This is a key-point of this paper and, using this fact, the main stability inequality follows by adapting a standard argument in the Riemannian setting; see, e.g. [31]. In Section 6 we prove the following:

Theorem 1.1. Let $S \subset \mathbb{G}$ be a H-minimal hypersurface of class $\mathbf{C}^{3}$. If there exists $\alpha \in I_{V}=\{h+1, \ldots, n\}$ such that either $\varpi_{\alpha}>0$ or $\varpi_{\alpha}<0$ on $S$, then each non-characteristic domain $\Omega \subset S$ is stable.

An immediate application of the previous result is contained in the next:

Corollary 1.2. Let $S \subset \mathbb{G}$ be a complete H-minimal hypersurface of class $\mathbf{C}^{3}$. If $S$ is a graph with respect to some given vertical direction, then each non-characteristic domain $\Omega \subset S$ is stable.

Finally, , in order to illustrate our results, an analysis of some (more or less simple) examples is given in Section 6.1, see, more precisely, Corollary 6.8, Corollary 6.9, and Corollary 6.12.

1.1. Carnot groups. A $k$-step Carnot group $(\mathbb{G}, \bullet)$ is a connected, simply connected, nilpotent and stratified Lie group (with respect to a group law $\bullet$ ) so that its Lie algebra $\mathfrak{g} \cong \mathbb{R}^{n}$ is a direct sum of slices $\mathfrak{g}=H_{1} \oplus \ldots \oplus H_{k}$ such that $\left[H_{1}, H_{i-1}\right]=H_{i} \quad(i=2, \ldots, k), H_{k+1}=\{0\}$. Let 0 be the identity of $\mathbb{G}$ and set $h_{i}:=\operatorname{dim} H_{i}$ for $i=1, \ldots, k$ and $h_{1}:=h$. Moreover set $H:=H_{1}$ and $V:=H_{2} \oplus \ldots \oplus H_{k}$. Note that $H$ and $V$ are smooth subbundles of $T \mathbb{G}$ called horizontal and vertical, respectively. The horizontal space $H$ is generated by a frame $X_{H}:=\left\{X_{1}, \ldots, X_{h}\right\}$ of left-invariant vector fields, which can be completed to a global graded, left-invariant frame $\underline{X}:=\left\{X_{1}, \ldots, X_{n}\right\}$ for $\mathfrak{g}$. We stress that the standard basis $\left\{\mathrm{e}_{i}: i=1, \ldots, n\right\}$ of $\mathbb{R}^{n} \cong T_{0} \mathbb{G}$ can be relabeled to be graded or adapted to the stratification. Note that any left-invariant vector field of $\underline{X}$ satisfies $X_{i}(x)=L_{x *} \mathrm{e}_{i}(i=1, \ldots, n)$, where $L_{x *}$ denotes the differential of the lefttranslation at $x \in \mathbb{G}$. We fix a Euclidean metric on $\mathbb{R}^{n} \cong T_{0} \mathbb{G}$ which makes $\left\{\mathrm{e}_{i}: i=1, \ldots, n\right\}$ an o.n. basis; this metric extends to each tangent space by left-translations and makes $\underline{X}$ an o.n. left-invariant frame for g. We denote by $g=\langle\cdot, \cdot\rangle$ this metric and assume that $(\mathbb{G}, g)$ is a Riemannian manifold.

We use the so-called exponential coordinates of 1st kind so that $\mathbb{G}$ is identified with its Lie algebra $\mathfrak{g}$, via the (Lie group) exponential map exp $: \mathfrak{g} \longrightarrow \mathbb{G}$.

A sub-Riemannian metric $g_{H}$ is a symmetric positive bilinear form on the horizontal space $H$. The $C C$-distance $d_{H}(x, y)$ between $x, y \in \mathbb{G}$ is given by

$$
d_{H}(x, y):=\inf \int \sqrt{g_{H}(\dot{\gamma}, \dot{\gamma})} d t
$$

where the infimum is taken over all piecewise-smooth horizontal paths $\gamma$ joining $x$ to $y$. From now on, we shall choose $g_{H}:=g_{\mid H}$.

We recall that Carnot groups are homogeneous groups, i.e. they admit a one-parameter group of automorphisms $\delta_{t}: \mathbb{G} \longrightarrow \mathbb{G}$ for any $t \geq 0$. By definition, one has $\delta_{t} x:=\exp \left(\sum_{j, i_{j}} t^{j} x_{i_{j}} \mathrm{e}_{i_{j}}\right)$, for every $x=\exp \left(\sum_{j, i_{j}} x_{i_{j}} \mathrm{e}_{i_{j}}\right) \in \mathbb{G}$. The homogeneous dimension of $\mathbb{G}$ is the integer $Q:=\sum_{i=1}^{k} i h_{i}$ coinciding with the Hausdorff dimension of ( $\left.\mathbb{G}, d_{H}\right)$ as a metric space; see [40], [58].

The structural constants of $\mathfrak{g}$ associated with $\underline{X}$ are defined by $C_{i j}^{g r}:=\left\langle\left[X_{i}, X_{j}\right], X_{r}\right\rangle, i, j, r=1, \ldots, n$. They are skew-symmetric and satisfy Jacobi's identity. The stratification hypothesis on $\mathfrak{g}$ can be restated as follows:

$$
X_{i} \in H_{l}, X_{j} \in H_{m} \Longrightarrow\left[X_{i}, X_{j}\right] \in H_{l+m}
$$

and so if $i \in I_{H_{s}}$ and $j \in I_{H_{r}}$, then

$$
C^{\mathrm{g} m} \neq 0 \Longrightarrow m \in I_{H_{s+r}} .
$$

We set

- $C_{H}^{\alpha}:=\left[C^{\mathrm{g} \alpha}{ }_{i j}\right]_{i, j=1, \ldots, h} \in \mathcal{M}_{h \times h}(\mathbb{R})$

- $C^{\alpha}:=\left[C_{i j}^{\mathrm{g} \alpha}\right]_{i, j=1, \ldots, n} \in \mathcal{M}_{n \times n}(\mathbb{R})$

$$
\begin{aligned}
& \forall \alpha=h+1, \ldots, h+h_{2} ; \\
& \forall \alpha=h+1, \ldots, n .
\end{aligned}
$$


Now we introduce $]^{4}$ the left-invariant co-frame $\underline{\omega}:=\left\{\omega_{i}: i=1, \ldots, n\right\}$ dual to $\underline{X}$, i.e. $\omega_{i}=X_{i}^{*} \in \Omega^{1}(\mathbb{G})$ for every $i=1, \ldots, n$. In particular, note that the left-invariant 1-forms $\omega_{i}$ are uniquely determined by

$$
\omega_{i}\left(X_{j}\right)=\left\langle X_{i}, X_{j}\right\rangle=\delta_{i}^{j} \quad \forall i, j=1, \ldots, n,
$$

where $\delta_{i}^{j}$ denotes the Kronecker delta.

Let $\nabla$ denote the (unique) left-invariant Levi-Civita connection on $\mathbb{G}$ associated with the left-invariant metric $g=\langle\cdot, \cdot\rangle$. It turns out that

$$
\nabla_{X_{i}} X_{j}=\frac{1}{2} \sum_{r=1}^{n}\left(C^{\mathrm{g} r}{ }_{i j}-C^{\mathrm{g} i}{ }_{j r}+C_{r i}^{\mathrm{g} j}\right) X_{r} \quad \forall i, j=1, \ldots, n .
$$

If $X, Y \in \mathfrak{X}(H):=\mathbf{C}^{\infty}(\mathbb{G}, H)$, we set $\nabla_{X}^{H} Y:=\mathcal{P}_{H}\left(\nabla_{X} Y\right)$. The operation $\nabla^{H}$ is a partial connection called $H$-connection. We stress that $\nabla^{H}$ is flat, compatible with the metric $g_{H}$ and torsion-free (i.e. $\left.\nabla_{X}^{H} Y-\nabla_{Y}^{H} X-\mathcal{P}_{H}[X, Y]=0 \forall X, Y \in \mathfrak{X}(H)\right)$; see [56] and references therein.

Notation 1.3. If $X \in \mathfrak{X}^{1}(T \mathbb{G}):=\mathbf{C}^{1}(\mathbb{G}, T \mathbb{G})$, we denote by $\mathcal{J}_{\mathcal{R}} X$ the Jacobian matrix of $X$ computed with respect to the left invariant frame $\underline{X}=\left\{X_{1}, \ldots, X_{n}\right\}$. Moreover, if $X \in \mathfrak{X}^{1}(H)=\mathbf{C}^{1}(\mathbb{G}, H)$, we denote by $\mathcal{J}_{H} X$ the horizontal Jacobian matrix of $X$ computed with respect to the horizontal left invariant frame $\underline{X_{H}}=\left\{X_{1}, \ldots, X_{h}\right\}$; see [56].

Remark 1.4 (Horizontal curvature tensor $\mathrm{R}_{H}$ ). The flatness of $\nabla^{H}$ implies that horizontal curvature tensor $\mathrm{R}_{H}$ is identically zero, where we recall that $\mathrm{R}_{H}(X, Y) Z:=\nabla_{Y}^{H} \nabla_{X}^{H} Z-\nabla_{X}^{H} \nabla_{Y}^{H} Z-\nabla_{[Y, X]_{H}}^{H} Z$ for $X, Y, Z \in \mathfrak{X}(H)$.

Horizontal gradient and horizontal divergence operators are denoted by $\operatorname{grad}_{H}$ and $\operatorname{div}_{H}$.

A continuous distance $\varrho: \mathbb{G} \times \mathbb{G} \longrightarrow \mathbb{R}_{+} \cup\{0\}$ is called homogeneous if one has

$$
\varrho(x, y)=\varrho(z \bullet x, z \bullet y) \quad \forall x, y, z \in \mathbb{G} ; \quad \varrho\left(\delta_{t} x, \delta_{t} y\right)=t \varrho(x, y) \quad \forall t>0 .
$$

We recall a fundamental example.

Example 1.5 (Heisenberg groups $\mathbb{H}^{n}$ ). The Lie algebra $\mathfrak{h}_{n} \cong \mathbb{R}^{2 n+1}$ of the $n$-th Heisenberg group can be defined by using a left-invariant frame $\left\{X_{1}, Y_{1}, \ldots, X_{i}, Y_{i}, \ldots, X_{n}, Y_{n}, T\right\}$, where $X_{i}(p):=\frac{\partial}{\partial x_{i}}-\frac{y_{i}}{2} \frac{\partial}{\partial t}$, $Y_{i}(p):=\frac{\partial}{\partial y_{i}}+\frac{x_{i}}{2} \frac{\partial}{\partial t}$ for every $i=1, \ldots, n$, and $T(p):=\frac{\partial}{\partial t}$. Here $p=\exp \left(x_{1}, y_{1}, x_{2}, y_{2}, \ldots, x_{n}, y_{n}, t\right)$ denotes the generic point in $\mathbb{H}^{n}$. One has $\left[X_{i}, Y_{i}\right]=T$ for every $i=1, \ldots, n$, and all other commutators vanish. Hence, by definition, $T$ is the center of $\mathfrak{h}_{n}$ and $\mathfrak{h}_{n}$ turns out to be nilpotent and stratified of step 2, i.e. $\mathfrak{h}_{n}=H \oplus H_{2}$. The structural constants of $\mathfrak{h}_{n}$ are described by the skew-symmetric $(2 n \times 2 n)$-matrix

$$
C_{H}^{2 n+1}:=\left|\begin{array}{ccccc}
0 & 1 & \cdot & 0 & 0 \\
-1 & 0 & \cdot & 0 & 0 \\
\cdot & \cdot & \cdot & . & \cdot \\
0 & 0 & \cdot & 0 & 1 \\
0 & 0 & \cdot & -1 & 0
\end{array}\right|
$$

associated with the skew-symmetric bilinear map $\Gamma_{H}: H \times H \longrightarrow \mathbb{R}$ given by $\Gamma_{H}(X, Y)=\langle[X, Y], T\rangle$ for every $X, Y \in H$.

1.2. Hypersurfaces and measures. The Riemannian left-invariant volume form on $\mathbb{G}$ is defined as $\sigma_{\mathcal{R}}^{n}:=\bigwedge_{i=1}^{n} \omega_{i} \in \Omega^{n}(\mathbb{G})$. The measure $\sigma_{\mathcal{R}}^{n}$ is the Haar measure of $\mathbb{G}$ and equals the push-forward of the usual $n$-dimensional Lebesgue measure $\mathcal{L}^{n}$ on $\mathbb{R}^{n} \cong T_{0} \mathbb{G}$.

Let $S \subset \mathbb{G}$ be a hypersurface of class $\mathbf{C}^{1}$. We say that $x \in S$ is a characteristic point whenever $\operatorname{dim} H_{x}=\operatorname{dim}\left(H_{x} \cap T_{x} S\right)$. The characteristic set of $S$ is defined as

$$
C_{S}:=\left\{x \in S: \operatorname{dim} H_{x}=\operatorname{dim}\left(H_{x} \cap T_{x} S\right)\right\} .
$$

Note that $x \in S$ is non-characteristic if, and only if, $H$ is transversal to $S$ at $x$, i.e. $H_{x} \pitchfork T_{x} S$. We here observe that the $(Q-1)$-dimensional CC Hausdorff measure of the characteristic set $C_{S}$ vanishes, i.e.

${ }^{4}$ Notation. Let $M$ be a smooth manifold. We shall denote by $\Omega^{k}(M)$ the space of differential $k$-forms on $M$. 
$\mathcal{H}_{C C}^{Q-1}\left(C_{S}\right)=0$; see [51]. In fact, under further regularity assumptions, it is possible to show much more than that. For instance, if $S$ is of class $\mathbf{C}^{2}$, then the $(n-1)$-dimensional Riemmanian Hausdorff measure of $C_{S}$ is zero; see [12].

Let $v$ denote the unit normal vector along $S$. The $(n-1)$-dimensional Riemannian measure is defined as $\left.\sigma_{\mathcal{R}}^{n-1}:=(v\lrcorner \sigma_{\mathcal{R}}^{n}\right)\left.\right|_{S}$, where $\lrcorner$ denotes the "contraction" operator on differential forms; see Lee's book [48], pp. 334-346. We recall that $\lrcorner: \Omega^{k}(\mathbb{G}) \rightarrow \Omega^{k-1}(\mathbb{G})$ is defined, for $X \in \mathfrak{X}(T \mathbb{G})$ and $\alpha \in \Omega^{k}(\mathbb{G})$, by setting

$$
(X\lrcorner \alpha)\left(Y_{1}, \ldots, Y_{k-1}\right):=\alpha\left(X, Y_{1}, \ldots, Y_{k-1}\right) .
$$

At each non-characteristic point of $S$ the unit $H$-normal along $S$ is the normalized projection of $v$ onto $H$, that is $v_{H}:=\frac{\mathcal{P}_{H} v}{\left|\mathcal{P}_{H} v\right|}$. The H-perimeter form is the $(n-1)$-differential form $\sigma_{H}^{n-1}$ on $S \backslash C_{S}$ defined by

$$
\left.\sigma_{H}^{n-1}:=\left(v_{H}\right\lrcorner \sigma_{\mathcal{R}}^{n}\right)\left.\right|_{S \backslash C_{S}} .
$$

If $C_{S} \neq \emptyset$ we extend $\sigma_{H}^{n-1}$ to the whole of $S$ by setting $\sigma_{H}^{n-1}\left\llcorner C_{S}=0\right.$. Note that $\sigma_{H}^{n-1}=\left|\mathcal{P}_{H} v\right| \sigma_{\mathcal{R}}^{n-1}$. This follows from the well-known formula $\left.(X\lrcorner \sigma_{\mathcal{R}}^{n}\right)\left.\right|_{S}=\langle X, v\rangle \sigma_{\mathcal{R}}^{n-1}$ for any $X \in \mathfrak{X}(T \mathbb{G})$. In particular, it turns out that $C_{S}=\left\{x \in S:\left|\mathcal{P}_{H} v(x)\right|=0\right\}$. Let $\mathcal{S}_{C C}^{Q-1}$ be the $(Q-1)$-dimensional spherical Hausdorff measure associated with the CC-distance $d_{H}$. Then $\sigma_{H}^{n-1}(S \cap B)=k\left(v_{H}\right) \mathcal{S}_{C C}^{Q-1}(S \cap B)$ for all $B \in \mathcal{B}$ or $(\mathbb{G})$, where the density $k\left(v_{H}\right)$, called metric factor, depends on $v_{H}$; see [50]. The horizontal tangent bundle $H S \subset T S$ and the horizontal normal bundle $v_{H} S$ split the horizontal bundle $H$ into an orthogonal direct sum, i.e. $H=v_{H} \oplus H S$. We also recall that the stratification of $\mathfrak{g}$ induces a stratification of $T S:=\oplus_{i=1}^{k} H_{i} S$, where $H S:=H_{1} S$; see [40].

Remark 1.6. We have $\operatorname{dim} H_{p} S=\operatorname{dim} H_{p}-1=h-1$ at each point $p \in S \backslash C_{S}$. Furthermore, note that the definition of $H S$ makes sense even if $p \in C_{S}$, but in such a case $\operatorname{dim} H_{p} S=\operatorname{dim} H_{p}=2 n$.

For the sake of simplicity, in the rest of this section we shall assume, unless otherwise mentioned, that $S \subset \mathbb{G}$ is a non-characteristic hypersurface of class $\mathbf{C}^{2}$. So let $\nabla^{T S}$ be the induced connection on $S$ from $\nabla$. The tangential connection $\nabla^{T S}$ induces a partial connection on $H S$ defined by

$$
\nabla_{X}^{H S} Y:=\mathcal{P}_{H S}\left(\nabla_{X}^{T S} Y\right) \quad \forall X, Y \in \mathfrak{X}^{1}(H S):=\mathbf{C}^{1}(S, H S) .
$$

It turns out that $\nabla_{X}^{H S} Y=\nabla_{X}^{H} Y-\left\langle\nabla_{X}^{H} Y, v_{H}\right\rangle v_{H}$. In the sequel, $H S$-gradient and $H S$-divergence will be denoted, respectively, by $\operatorname{grad}_{H S}$ and $\operatorname{div}_{H S}$. By definition, the horizontal 2nd fundamental form of $S$ is the bilinear map given by

$$
B_{H}(X, Y):=\left\langle\nabla_{X}^{H} Y, v_{H}\right\rangle
$$

for any $X, Y \in \mathfrak{X}^{1}(T S)$. The horizontal mean curvature $\mathcal{H}_{H}$ is the trace of $B_{H}$, i.e. $\mathcal{H}_{H}:=\operatorname{Tr} B_{H}=-\operatorname{div} v_{H}$. The torsion $\mathrm{T}_{H S}$ of the $H S$-connection $\nabla^{H S}$ is given by $\mathrm{T}_{H S}(X, Y):=\nabla_{X}^{H S} Y-\nabla_{Y}^{H S} X-\mathcal{P}_{H}[X, Y]$ for any $X, Y \in \mathfrak{X}^{1}(H S)$. There is a non-zero torsion because, in general, $B_{H}$ is not symmetric. Hence $B_{H}$ can be regarded as a sum of two matrices, i.e. $B_{H}=S_{H}+A_{H}$, where $S_{H}$ is symmetric and $A_{H}$ is skew-symmetric.

Definition 1.7. The principal horizontal curvatures $\kappa_{j}$ of $S, j \in I_{H S}$, are the eigenvalues of $S_{H}$, i.e. eigenvalues of the symmetric part of the horizontal 2 nd fundamental form $B_{H}$. Note that $\mathcal{H}_{H}=\sum_{j \in I_{H S}} \kappa_{j}$. We also define some important geometric objects:

- $\varpi_{\alpha}:=\frac{v_{\alpha}}{\left|\mathcal{P}_{H} v\right|} \quad \forall \alpha=h+1, \ldots, n$;

- $\varpi_{H_{2}}:=\frac{\mathcal{P}_{H_{2}} v}{\left|\mathcal{P}_{H} v\right|}=\sum_{\alpha \in I_{H_{2}}} \varpi_{\alpha} X_{\alpha}$;

- $\varpi:=\frac{\mathcal{P}_{V} v}{\left|\mathcal{P}_{H} v\right|}=\sum_{\alpha \in I_{V}} \varpi_{\alpha} X_{\alpha}$;

- $C_{H}\left(\varpi_{H_{2}}\right):=\sum_{\alpha \in I_{H_{2}}} \varpi_{\alpha} C_{H}^{\alpha}$;

- $C(\varpi):=\sum_{\alpha \in I_{V}} \varpi_{\alpha} C^{\alpha}$.

We further denote by $C_{H S}\left(\varpi_{H_{2}}\right)$ the restriction to the subspace $H S$ of the linear operator $C_{H}\left(\varpi_{H_{2}}\right)$. 
These objects play an important role in the horizontal geometry of immersed hypersurfaces. For instance, we have to remark that $A_{H}=\frac{1}{2} C_{H S}\left(\varpi_{H_{2}}\right)$; see [56]. Moreover, for any $X, Y \in \mathfrak{X}^{1}(H S)$ we have $\mathrm{T}_{H S}(X, Y)=\langle[X, Y], \varpi\rangle \nu_{H}=-\left\langle C_{H S}\left(\varpi_{H_{2}}\right) X, Y\right\rangle$.

Example 1.8 (Heisenberg group). We have $\varpi:=\varpi_{T}=\frac{\langle v, T\rangle}{\left|\mathcal{P}_{H} v\right|}$ and $C_{H}\left(\varpi_{H_{2}}\right)=\varpi C_{H}^{2 n+1}$; see Example 1.5 An elementary computation shows that the skew-symmetric part $A_{H}$ of the horizontal 2nd fundamental form $B_{H}$ is given by $A_{H}=\frac{\pi}{2} C_{H S}^{2 n+1}$, where $C_{H S}^{2 n+1}=\left.C_{H}^{2 n+1}\right|_{H S}$. Since $\left\|C_{H S}^{2 n+1}\right\|_{G r}^{2}=2(n-1)$, it follows that $\left\|B_{H}\right\|_{\mathrm{G} r}^{2}=\left\|S_{H}\right\|_{\mathrm{G} r}^{2}+\frac{n-1}{2} \varpi^{2}$.

Definition 1.9. Let $U \subseteq \mathbb{G}$ be an open set and let $\mathcal{U}:=S \cap U$. We call adapted frame to $\mathcal{U}$ on $U$ any o.n. frame $\underline{\tau}:=\left\{\tau_{1}, \ldots, \tau_{n}\right\}$ on $U$ such that $\tau_{1}(p):=v_{H}(p), H_{p} \mathcal{U}=\operatorname{span}\left\{\tau_{2}(p), \ldots, \tau_{h}(p)\right\}$ for any $p \in \mathcal{U}$, $\tau_{\alpha}:=X_{\alpha}$. Furthermore, we set $\tau_{\alpha}^{T S}:=\tau_{\alpha}-\varpi_{\alpha} \tau_{1}$ for any $\alpha \in I_{V}$. We stress that $H S^{\perp}=\operatorname{span}_{\mathbb{R}}\left\{\tau_{\alpha}^{T S}: \alpha \in I_{V}\right\}$, where $H S^{\perp}$ denotes the orthogonal complement of $H S$ in $T S$, i.e. $T S=H S \oplus H S^{\perp}$.

Note that every adapted o.n. frame to a hypersurface is a graded frame. Clearly, we have that

$$
\underline{\tau}=\{\underbrace{\tau_{1}}_{=v_{H}}, \underbrace{\tau_{2}, \ldots, \tau_{h}}_{\text {o.n. basis of } H S}, \underbrace{\tau_{h+1}, \ldots, \tau_{n}}_{\text {o.n. basis of } V}\} .
$$

Notation 1.10. Let $n_{i}:=\sum_{j=1}^{i} h_{j}$. Hereafter, we shall set $I_{H}=\{1,2, \ldots, h\}, I_{H_{i}}=\left\{n_{i-1}+1, \ldots, n_{i}\right\}$, $I_{V}=\{h+1, \ldots, n\}$ and $I_{H S}:=\{2,3, \ldots, h\}$.

Let $\phi:=\left\{\phi_{1}, \ldots, \phi_{n}\right\}$ be the dual co-frame of $\underline{\tau}$, i.e. $\phi_{i}\left(\tau_{j}\right)=\delta_{i}^{j}$ for any $i, j=1, \ldots, n$, where $\delta_{i}^{j}$ denotes the Kroneker delta. The co-frame $\phi$ satisfies the Cartan's structural equations:

$$
\text { (I) } d \phi_{i}=\sum_{j=1}^{n} \phi_{i j} \wedge \phi_{j}, \quad \text { (II) } d \phi_{j k}=\sum_{l=1}^{n} \phi_{j l} \wedge \phi_{l k}-\Phi_{j k}
$$

for any $i, j, k=1, \ldots, n$, where $\phi_{i j}(X):=\left\langle\nabla_{X} \tau_{j}, \tau_{i}\right\rangle$ denote the connection 1-forms of $\phi$ and $\Phi_{j k}$ denote the curvature 2-forms, defined by $\Phi_{j k}(X, Y):=\phi_{k}\left(\mathrm{R}(X, Y) \tau_{j}\right)$ for any $X, Y \in \mathfrak{X}(\mathbb{G})$, where $\mathrm{R}$ is the Riemannian curvature tensor, that is

$$
\mathrm{R}(X, Y) Z:=\nabla_{Y} \nabla_{X} Z-\nabla_{X} \nabla_{Y} Z-\nabla_{[Y, X]} Z
$$

for any $X, Y, Z \in \mathfrak{X}(\mathbb{G})$. The following holds

$$
C_{i j}^{k}:=\left\langle\left[\tau_{i}, \tau_{j}\right], \tau_{k}\right\rangle=\phi_{j k}\left(\tau_{i}\right)-\phi_{i k}\left(\tau_{j}\right) \quad \forall i, j, k=1, \ldots, n .
$$

This identity can be proved by using the fact that $\nabla$ is torsion-free.

Definition 1.11. A vertical hyperplane $\mathcal{I}$ is the zero-set of a linear homogeneous polynomial on $\mathbb{G}$ of homogeneous degree 1. A non-vertical hyperplane $\mathcal{I}$ is the zero-set of a linear polynomial on $\mathbb{G}$ of homogeneous degree greater than or equal to 2 .

Hyperplanes are $(n-1)$-dimensional vector subspaces of $\mathfrak{g}$. The importance of vertical hyperplanes comes from the intrinsic rectifiability theory developed by Franchi, Serapioni and Serra Cassano in 2step Carnot groups; see [32, 33, 34, 35]. They turn out to be ideals of the Lie algebra $\mathfrak{g}$ and may be thought of as generalized tangent spaces to sets of finite $H$-perimeter (in the variational sense); see [6]. Non-vertical hyperplanes will be studied in Section 6.1

\section{DiVERGenCe formulae}

Let $S \subset \mathbb{G}$ be a hypersurface of class $\mathbf{C}^{2}$. For the sake of simplicity, we first assume that $S$ is noncharacteristic. Let $\mathbf{C}_{H S}^{i}(S),(i=1,2)$ be the space of functions whose $H S$-derivatives up to the $i$-th order are continuous on $S$. Analogously, for any open subset $\mathcal{U} \subseteq S$, we set $\mathbf{C}_{H S}^{i}(\mathcal{U})$, to denote the space of functions whose $H S$-derivatives up to the $i$-th order are continuous on $\mathcal{U}$. The previous definitions extend to the case $C_{S} \neq \emptyset$ by requiring that all $H S$-derivatives up to the $i$-th order are continuous on $C_{S}$. 
Remark 2.1. The notions concerning the $H S$-connection $\nabla^{H S}$, the horizontal 2 nd fundamental form $B_{H}$ and the torsion $\mathrm{T}_{H S}$, can also be formulated by replacing $\mathfrak{X}^{1}(H S)=\mathbf{C}^{1}(S, H S)$ with the larger space $\mathfrak{X}_{H S}^{1}(H S):=\mathbf{C}_{H S}^{1}(S, H S)$.

Definition 2.2 (HS-differential operators). Let $\mathcal{D}_{H S}: \mathfrak{X}_{H S}^{1}(H S) \longrightarrow \mathbf{C}(S)$ be the 1st order differential operator given by

$$
\mathcal{D}_{H S} X:=\operatorname{div}_{H S} X+\left\langle C_{H}\left(\varpi_{H_{2}}\right) v_{H}, X\right\rangle \quad \forall X \in \mathfrak{X}_{H S}^{1}(H S) .
$$

Moreover, let $\mathcal{D}_{H S}: \mathbf{C}_{H S}^{2}(S) \longrightarrow \mathbf{C}(S)$ be the 2nd order differential operator defined as

$$
\mathcal{L}_{H S} \varphi:=\Delta_{H S} \varphi+\left\langle C_{H}\left(\varpi_{H_{2}}\right) v_{H}, \operatorname{grad}_{H S} \varphi\right\rangle \quad \forall \varphi \in \mathbf{C}_{H S}^{2}(S) .
$$

Note that $\mathcal{D}_{H S}(\varphi X)=\varphi \mathcal{D}_{H S} X+\left\langle\operatorname{grad}_{H S} \varphi, X\right\rangle$ for every $X \in \mathfrak{X}^{1}(H S)$ and every $\varphi \in \mathbf{C}_{H S}^{1}(S)$. Moreover $\mathcal{L}_{H S} \varphi=\mathcal{D}_{H S}\left(\operatorname{grad}_{H S} \varphi\right)$ for every $\varphi \in \mathbf{C}_{H S}^{2}(S)$.

It is not difficult to see that the operators $\Delta_{H S}$ and $\mathcal{L}_{H S}$ naturally extend to horizontal vector fields. These extensions will be denoted by $\overrightarrow{\Delta_{H S}}$ and $\overrightarrow{\mathcal{L}_{H S}}$. We remark that

$$
\overrightarrow{\mathcal{L}_{H S}} X=\overrightarrow{\Delta_{H S}} X+\left(\mathcal{J}_{H S} X\right) C_{H}\left(\varpi_{H_{2}}\right) v_{H}
$$

for every $X \in \mathbf{C}_{H S}^{2}\left(S \backslash C_{S}, H S\right)$, where $\mathcal{J}_{H S} X$ denotes the $H S$-Jacobian matrix of the horizontal tangent vector field $X$.

We now define a homogeneous measure $\sigma_{H}^{n-2}$, which plays the role of the intrinsic Hausdorff measure on $(n-2)$-dimensional submanifolds of $\mathbb{G}$.

Remark 2.3 (The measure $\sigma_{H}^{n-2}$ ). Let $\eta \in \mathfrak{X}(T S)$ be a unit normal vector orienting $\partial S$. Furthermore, let $\eta_{H S}:=\frac{\mathcal{P}_{H S} \eta}{\mid \mathcal{P}_{H S} \eta}$ be the unit $H S$-normal of $\partial S$. By definition, we set $\left.\sigma_{H}^{n-2}:=\left(\eta_{H S}\right\lrcorner \sigma_{H}^{n-1}\right)\left.\right|_{\partial S}$. Exactly as for the H-perimeter $\sigma_{H}^{n-1}$, the measure $\sigma_{H}^{n-2}$, which is $(Q-2)$-homogeneous with respect to Carnot dilations, can be represented in terms of the Riemannian measure $\sigma_{\mathcal{R}}^{n-2}$. In fact, we have $\sigma_{H}^{n-2}=\left|\mathcal{P}_{H} v\right|\left|\mathcal{P}_{H S} \eta\right| \sigma_{\mathcal{R}}^{n-2}\llcorner\partial S$.

The above definitions are somehow motivated by Theorem 3.17 in [56].

Theorem 2.4. Let $S \subset \mathbb{G}$ be a compact non-characteristic hypersurface of class $\mathbf{C}^{2}$ with (piecewise) $\mathbf{C}^{1}$ boundary $\partial S$ and $X \in \mathfrak{X}^{1}(T S)$. Then

$$
\int_{S} \mathcal{D}_{H S} X \sigma_{H}^{n-1}=\int_{\partial S}\left\langle X, \eta_{H S}\right\rangle \sigma_{H}^{n-2}
$$

As a consequence, the following integral formula holds

$$
\int_{S} \mathcal{D}_{H S} X \sigma_{H}^{n-1}=\int_{S}\left(\operatorname{div}_{H S} X+\left\langle C_{H}\left(\varpi_{H_{2}}\right) v_{H}, X\right\rangle\right) \sigma_{H}^{n-1}=0
$$

for every $X \in \mathbf{C}_{0}^{1}(S, H S)$.

Stokes' formula is concerned with integrating a $k$-form over a $k$-dimensional manifold with boundary. A common way to state this fundamental result is the following.

Proposition 2.5. Let $M$ be an oriented $k$-dimensional manifold of class $\mathbf{C}^{2}$ with boundary $\partial M$. Then $\int_{M} d \alpha=\int_{\partial M} \alpha$ for every compactly supported $(k-1)$-form $\alpha$ of class $\mathbf{C}^{1}$.

One requires $M$ to be of class $\mathbf{C}^{2}$ for a technical reason concerning "pull-back" of differential forms. We remark that it is possible to extend Proposition 2.5 to the following cases:

( $\star \bar{M}$ is of class $\mathbf{C}^{1}$ and $\alpha$ is a $(k-1)$-form such that $\alpha$ and $d \alpha$ are continuous;

(ه) $\bar{M}$ is of class $\mathbf{C}^{1}$ and $\alpha$ is a $(k-1)$-form such that $\alpha \in L^{\infty}(M)$, d $\alpha \in L^{1}(M)$-or $d \alpha \in L^{\infty}(M)$ - and $\imath_{M}^{*} \alpha \in L^{\infty}(\partial M)$, where $\imath_{M}: \partial M \longrightarrow \bar{M}$ is the natural inclusion.

Many different versions of Stokes' theorem are available in literature; see, for instance, [29]. For an introduction, we refer the reader to the book by Taylor [75]; see Appendix G. 
Remark 2.6. General versions of Stokes' theorem can be deduced from the generalized Gauss-Green formula proved by De Giorgi and Federer; see [27] or [29], Theorem 4.5.6, p. 478. However, it is worth observing that they hold for Lipschitz differential forms. A general result of this type can be found in Maz'ja' [53], see Section 6.2. We observe that ( $\star$ ) holds for any compact oriented $k$-dimensional manifold $M$ with boundary and for differential forms that are Lipschitz at each point of $M \backslash T$, where $T$ is a thin subset of M; see [65], Remark 5.3.2, p. 197. On the other hand ( $\$$ ) is perhaps less known that ( $\star$ ). The validity of $(\mathbf{\star})$ is observed in [75]; see formula (G.38), Appendix G. This result can be deduced by applying a standard procedur 5 from a divergence-type theorem proved by Anzellotti; see, more precisely, Theorem 1.9 in [7]. More recent and more general results can also be found in the paper by Chen, Torres and Ziemer [20].

We have here to remark that either condition $(\star)$ or $(\star)$ can be used to extend the horizontal integration by parts formulae to vector fields (and functions) possibly singular at the characteristic set $C_{S}$.

Definition 2.7. Let $X \in \mathbf{C}^{1}\left(S \backslash C_{S}, H S\right)$ and set $\left.\alpha_{X}:=(X\lrcorner \sigma_{H}^{n-1}\right)\left.\right|_{S}$. We say that $X$ is admissible (for the horizontal divergence formula) if the differential forms $\alpha_{X}$ and $d \alpha_{X}$ satisfy either condition ( $\star$ ) or ( $(\star)$ on $S$. We say that $\phi \in \mathbf{C}_{H S}^{2}\left(S \backslash C_{S}\right)$ is admissible if $\operatorname{grad}_{H S} \phi$ is admissible for the horizontal divergence formula. More generally, let $X \in \mathbf{C}^{1}\left(S \backslash C_{S}, T S\right)$ and set $\left.\alpha_{X}:=(X\lrcorner \sigma_{H}^{n-1}\right)\left.\right|_{S}$. Then, we say that $X$ is admissible (for the Riemannian divergence formula) whenever $\alpha_{X}$ and d $\alpha_{X}$ satisfy either condition ( $\star$ ) or (๑) on $S$.

Using Definition 2.7 and Theorem 2.4 yields the following:

Corollary 2.8. Let $S \subset \mathbb{G}$ be a compact hypersurface of class $\mathbf{C}^{2}$ with $\mathbf{C}^{1}$ boundary $\partial S$. We have:

(i) $\int_{S} \mathcal{D}_{H S} X \sigma_{H}^{n-1}=\int_{\partial S}\left\langle X, \eta_{H S}\right\rangle \sigma_{H}^{n-2}$ for every admissible $X \in \mathbf{C}^{1}\left(S \backslash C_{S}, H S\right)$;

(ii) $\int_{S} \mathcal{L}_{H S} \phi \sigma_{H}^{n-1}=\int_{\partial S}\left\langle\operatorname{grad}_{H S} \phi, \eta_{H S}\right\rangle \sigma_{H}^{n-2}$ for every admissible $\phi \in \mathbf{C}_{H S}^{2}\left(S \backslash C_{S}\right)$;

(iii) if $\partial S=\emptyset$, then

$$
-\int_{S} \varphi \mathcal{L}_{H S} \varphi \sigma_{H}^{n-1}=\int_{S}\left|\operatorname{grad}_{H S} \varphi\right|^{2} \sigma_{H}^{n-1}
$$

for every function $\varphi \in \mathbf{C}_{H S}^{2}\left(S \backslash C_{S}\right)$ such that $\varphi^{2}$ is admissible.

Note that formula 6 holds even if $\partial S \neq \emptyset$, but in this case we have to use compactly supported functions on $S$.

Remark 2.9. Let $\varphi \in \mathbf{C}_{H S}^{2}\left(S \backslash C_{S}\right)$. If $\varphi^{2}$ is admissible, then

$$
\varphi \in W_{H S}^{1,2}\left(S, \sigma_{H}^{n-1}\right)=\left\{\varphi \in L^{2}\left(S, \sigma_{H}^{n-1}\right):\left|\operatorname{grad}_{H S} \varphi\right| \in L^{2}\left(S, \sigma_{H}^{n-1}\right)\right\} .
$$

Example 2.10 (Heisenberg group; see Example 1.8). One has $\mathcal{D}_{H S}(X):=\operatorname{div}_{H S} X+\varpi\left\langle C_{H}^{2 n+1} v_{H}, X\right\rangle$ for every $X \in \mathfrak{X}^{1}(H S)$ and $\mathcal{L}_{H S} \varphi:=\mathcal{D}_{H S}\left(\operatorname{grad}_{H S} \varphi\right)=\Delta_{H S} \varphi+\varpi\left\langle C_{H}^{2 n+1} v_{H}, \operatorname{grad}_{H S} \varphi\right\rangle$ for every $\varphi \in \mathbf{C}_{H S}^{2}(S)$.

The following result will be used throughout the proof of Corollary 4.13

Proposition 2.11. Let $M$ be a compact oriented Lipschitz $k$-dimensional Riemannian manifold. Then $\int_{M} \operatorname{div}_{T M} X \sigma_{\mathcal{R}}^{k}=0$ for every $X \in W_{\text {comp }}^{1,2}(M, T M)$, where $\sigma_{\mathcal{R}}^{k}$ is the Riemannian volume and $W_{\text {comp }}^{1,2}(M)$ denotes the Sobolev space of all square-integrable compactly supported vector fields on $M$ with squareintegrable first (covariant) derivative.

This propoposition can be found in [69]; see Proposition 3.1, p. 7; see also [68], Section 4.3, p. 33.

\footnotetext{
${ }^{5}$ See, for instance, Federer [29], paragraph 3.2.46, p. 280; see also [65], Remark 5.3.2, p. 197.
} 


\subsection{Preliminary remarks concerning the 1 st variation formula.}

Notation 2.12. Let $S \subset \mathbb{G}$ be a hypersurface of class $\mathbf{C}^{i}, i \geq 2$. Let $X \in T \mathbb{G}$ and let $v$ be the outwardpointing unit normal vector along $S$. We shall denote by $X^{\perp}$ and $X^{\top}$ the standard decomposition of $X$ into its normal and tangential components, i.e. $X^{\perp}=\langle X, v\rangle v, X^{\top}=X-X^{\perp}$.

We now make a simple (but fundamental) calculation.

Lemma 2.13. If $X \in \mathfrak{X}^{1}(T \mathbb{G})$, then $\left.\left.(X\lrcorner \sigma_{H}^{n-1}\right)\left.\right|_{S}=\left(\left(X^{\top}\left|\mathcal{P}_{H} v\right|-\langle X, v\rangle v_{H}^{\top}\right)\right\lrcorner \sigma_{\mathcal{R}}^{n-1}\right)\llcorner S$. Moreover, at each non-characteristic point of $S$, we have

$$
\left.d(X\lrcorner \sigma_{H}^{n-1}\right)\left.\right|_{S}=\operatorname{div}_{T S}\left(X^{\top}\left|\mathcal{P}_{H} v\right|-\langle X, v\rangle v_{H}^{\top}\right) \sigma_{\mathcal{R}}^{n-1}\llcorner S .
$$

Proof. We have

$$
\begin{aligned}
\left.d(X\lrcorner \sigma_{H}^{n-1}\right)\left.\right|_{S} & \left.\left.=(X\lrcorner v_{H}\right\lrcorner \sigma_{\mathcal{R}}^{n}\right)\left.\right|_{S} \\
& \left.\left.=d\left(\left(X^{\top}+X^{\perp}\right)\right\lrcorner\left(v_{H}^{\top}+v_{H}^{\perp}\right)\right\lrcorner \sigma_{\mathcal{R}}^{n}\right)\left.\right|_{S} \\
& \left.\left.\left.\left.=d\left(X^{\top}\right\lrcorner v_{H}^{\perp}\right\lrcorner \sigma_{\mathcal{R}}^{n}\right)\left.\right|_{S}+d\left(v_{H}^{\top}\right\lrcorner X^{\perp}\right\lrcorner \sigma_{\mathcal{R}}^{n}\right)\left.\right|_{S} \\
& \left.\left.=d\left(X^{\top}\right\lrcorner \sigma_{H}^{n-1}\right)\left.\right|_{S}+d\left(v_{H}^{\top}\right\lrcorner\langle X, v\rangle \sigma_{\mathcal{R}}^{n-1}\right)\left.\right|_{S} \\
& =d i v_{T S}\left(X^{\top}\left|\mathcal{P}_{H} v\right|-\langle X, v\rangle v_{H}^{\top}\right) \sigma_{\mathcal{R}}^{n-1}\llcorner S .
\end{aligned}
$$

Remark 2.14. The previous calculation corrects a mistake in [56], where the normal component of the vector field $X$ was omitted. This has caused the loss of some divergence-type terms in some of the variational formulae proved there.

We would like to stress that the importance of the previous calculation in the development of this paper comes from the well-known Cartan's identity for the Lie derivative of a differential form; see [15], [48]. More precisely, let $M$ be a smooth manifold, let $\omega \in \Omega^{k}(M)$ be a differential $k$-form on $M$ and let $X \in \mathfrak{X}(T M)$ be a differentiable vector field on $M$, with associated flow $\phi_{t}: M \longrightarrow M$. We recall that the Lie derivative of $\omega$ with respect to $X$, is defined by $\mathcal{L}_{X} \omega:=\left.\frac{d}{d t} \phi_{t}^{*} \omega\right|_{t=0}$, where $\phi_{t}^{*} \omega$ denotes the pull-back of $\omega$ by $\phi_{t}$. Then, Cartan's identity says that

$$
\left.\left.\mathcal{L}_{X} \omega=(X\lrcorner d \omega\right)+d(X\lrcorner \omega\right) .
$$

This is a very useful tool in proving variational formulae, not only for the case of Riemannian volume forms, for which we refer the reader to Spivak's book [72] (see Ch. 9, pp. 411-426 and 513-535), but even for more general functionals; see, for instance, [41], [39]. In Section 4] we shall apply this method to write down the 1 st and 2 nd variation formulae for the $H$-perimeter measure $\sigma_{H}^{n-1}$. But let us say something more about the 1 st variation formula. So let $S \subset \mathbb{G}$ be a hypersurface of class $\mathbf{C}^{2}$. We remark that the Lie derivative of $\sigma_{H}^{n-1}$ with respect to $X$ can be calculated elementarily as follows. We begin with the first term in formula (7). We have

$$
\left.\left.\left.X\lrcorner d \sigma_{H}^{n-1}=X\right\lrcorner d\left(v_{H}\right\lrcorner \sigma_{\mathcal{R}}^{n}\right)=X\right\lrcorner\left(\operatorname{div} v_{H} \sigma_{\mathcal{R}}^{n}\right)=\langle X, v\rangle \operatorname{div} v_{H} \sigma_{\mathcal{R}}^{n-1} .
$$

Note that $\operatorname{div} v_{H}=\operatorname{div} v_{H} v_{H}=-\mathcal{H}_{H}$. More precisely

$$
\operatorname{div} v_{H}=\sum_{i=1}^{n}\left\langle\nabla_{X_{i}} v_{H}, X_{i}\right\rangle=\sum_{i=1}^{h} X_{i}\left(v_{H i}\right)=\operatorname{div}_{H} v_{H}=-\mathcal{H}_{H} .
$$

The second term in formula (7) has been already computed in Lemma 2.13, Thus, we can conclude that

$$
\mathcal{L}_{X} \sigma_{H}^{n-1}=\left(-\mathcal{H}_{H}\langle X, v\rangle+\operatorname{div}_{T S}\left(X^{\top}\left|\mathcal{P}_{H} v\right|-\langle X, v\rangle v_{H}^{\top}\right)\right) \sigma_{\mathcal{R}}^{n-1},
$$

at each non-characteristic point of $S$. We will return on this point in Section 4 
Remark 2.15. Roughly speaking, formula (8) gives the "infinitesimal" 1 st variation of the measure $\sigma_{H}^{n-1}$. However, in order to integrate the function $\mathcal{L}_{X} \sigma_{H}^{n-1}$ over a $\mathbf{C}^{2}$ hypersurface $S$, we have to require that $\mathcal{H}_{H}$ is locally integrable on $S$, with respect to the Riemannian measure $\sigma_{\mathcal{R}}^{n-1}$, i.e.

$$
\mathcal{H}_{H} \in L_{l o c}^{1}\left(S ; \sigma_{\mathcal{R}}^{n-1}\right) .
$$

Indeed, in general, $\mathcal{H}_{H}$ fails to be integrable locally around the characteristic $C_{S}$; see, for instance, [26]. Note that (9) implies the integrability of the function $\mathcal{L}_{X} \sigma_{H}^{n-1}$. Clearly, if $C_{S}=\emptyset$, then (9) is automatically satisfied because, if $S$ is of class $\mathbf{C}^{2}$, then $\mathcal{H}_{H} \in \mathbf{C}(S)$.

Remark 2.16 (Riemannian case). We would like to stress the analogy with the 1st variation of $\sigma_{\mathcal{R}}^{n-1}$ for a hypersurface $S$ of class $\mathbf{C}^{i}, i \geq 1$, immersed in the Euclidean space $\mathbb{R}^{n}$. It is well-known that the 1st variation formula is given by $I_{S}\left(\sigma_{\mathcal{R}}^{n-1}\right)=\int_{S} d i v_{T S} W \sigma_{\mathcal{R}}^{n-1}$; see Simon's book [70], Ch. 2, § 9, pp. 48-53. In the $\mathbf{C}^{1}$-case, the variation vector $W$ cannot be decomposed in its normal and tangential parts. Clearly, this can be done if $S$ is of class $\mathbf{C}^{2}$. In this case

$$
I_{S}\left(\sigma_{\mathcal{R}}^{n-1}\right)=\int_{S} \operatorname{div}_{T S} W \sigma_{\mathcal{R}}^{n-1}=\int_{S}\left(\left\langle W^{\perp}, v\right\rangle \operatorname{div}_{T S} v+\operatorname{div}_{T S} W^{\top}\right) \sigma_{\mathcal{R}}^{n-1} .
$$

Note that $-\mathcal{H}_{\mathcal{R}}=\operatorname{div}_{T S} v$. Hence, we have two contributions. The first is given by $-\int_{S} \mathcal{H}_{\mathcal{R}}\left\langle W^{\perp}, v\right\rangle \sigma_{\mathcal{R}}^{n-1}$ and only depends on the normal component of the variation vector $W$. By means of the divergence theorem, the second term can be transformed in a boundary integrat given by $\int_{\partial S}\left\langle W^{\top}, \eta\right\rangle \sigma_{\mathcal{R}}^{n-2}$ which really depends only on the tangential component of $W$.

Remark 2.17 (Horizontal variations). Let $S \subset \mathbb{G}$ be a compact hypersurface of class $\mathbf{C}^{2}$ and $v$ the outward-pointing unit normal vector along $S$. We observe that formula (5) generalizes to the following:

$$
\int_{S} \mathcal{D}_{H S} X \sigma_{H}^{n-1}=-\int_{S} \mathcal{H}_{H}\left\langle X, v_{H}\right\rangle \sigma_{H}^{n-1}+\int_{\partial S}\left\langle X, \eta_{H S}\right\rangle \sigma_{H}^{n-2} \quad \forall X \in \mathfrak{X}^{1}(H)
$$

see, for instance, Corollary 3.19 and Theorem 4.3 in [56]. Note that (10) holds if either $C_{S}=\emptyset$ or, if $C_{S} \neq \emptyset$, whenever $X \in \mathbf{C}^{1}\left(S \backslash C_{S}, H\right)$ is an admissible vector field.

Formula (10) can be seen as a particular case of the 1 st variation formula of the $H$-perimeter; see formula (24) in Theorem 4.6 below. Actually, note that if $X=X_{H} \in \mathfrak{X}(H)$, then

$$
\begin{aligned}
X_{H}^{\top}\left|\mathcal{P}_{H} v\right|-\left\langle X_{H}^{\perp}, v\right\rangle v_{H}^{\top} & =\left(X_{H}-\left|\mathcal{P}_{H} v\right|\left\langle X_{H}, v_{H}\right\rangle v\right)\left|\mathcal{P}_{H} v\right|-\left|\mathcal{P}_{H} v\right|\left\langle X_{H}, v\right\rangle\left(v_{H}-\left|\mathcal{P}_{H} v\right| v\right) \\
& =\left(X_{H}-\left\langle X_{H}, v\right\rangle v_{H}\right)\left|\mathcal{P}_{H} v\right| \quad\left(=: X_{H S}\left|\mathcal{P}_{H} v\right|\right),
\end{aligned}
$$

where we have used the fact that $v=\left|\mathcal{P}_{H} v\right| v_{H}+\sum_{\alpha \in I_{V}} v_{\alpha} X_{\alpha}$ at each non-characteristic point. Now inserting this into (8) yields

$$
\begin{aligned}
\mathcal{L}_{X_{H}} \sigma_{H}^{n-1} & =\left(-\mathcal{H}_{H}\left\langle X_{H}, v\right\rangle+\operatorname{div}_{T S}\left(X_{H S}\left|\mathcal{P}_{H} v\right|\right)\right) \sigma_{\mathcal{R}}^{n-1} \\
& =-\mathcal{H}_{H}\left\langle X_{H}, v_{H}\right\rangle \sigma_{H}^{n-1}+\operatorname{div}_{T S}\left(X_{H S}\left|\mathcal{P}_{H} v\right|\right) .
\end{aligned}
$$

Hence, integrating this expression along $S$ and using Corollary 2.8, the claim follows.

\section{SOME TECHNICAL PRELIMINARIES ABOUT THE CONNECTION 1-FORMS}

Let $S \subset \mathbb{G}$ be a hypersurface of class $\mathbf{C}^{2}$ and let $U \subset \mathbb{G}$ be an open set having non-empty intersection with $S$ and such that $\mathcal{U}:=U \cap S$ is non-characteristic. We start with an elementary calculation.

Lemma 3.1. We have div $v_{T S} v_{H}=-\mathcal{H}_{H}-\left\langle C\left(\mathcal{P}_{V}\right) v_{H}, \mathcal{P}_{V} v\right\rangle$, where $C\left(\mathcal{P}_{V}\right):=\sum_{\alpha \in I_{V}} v_{\alpha} C^{\alpha}$.

\footnotetext{
${ }^{6}$ In this case, we further assume that $\partial S$ is a $(n-2)$-dimensional submanifold of class $\mathbf{C}^{1}$ oriented by the outward unit normal vector $\eta$.
} 
Proof. We have $\operatorname{div} v_{T S} v_{H}=\operatorname{div} v_{H}-\left\langle\nabla_{v} v_{H}, v\right\rangle$. Since $\operatorname{div} v_{H}=-\mathcal{H}_{H}$, the thesis follows from

$$
\left\langle\nabla_{\nu} v_{H}, v\right\rangle=\sum_{j \in I_{H}} \sum_{\alpha, \beta \in I_{V}} v_{H j} v_{\alpha} v_{\beta}\left\langle\nabla_{X_{\alpha}} X_{j}, X_{\beta}\right\rangle=\sum_{j \in I_{H}} \sum_{\alpha, \beta \in I_{V}} v_{H} v_{\alpha} v_{\beta} \frac{\left(C_{\alpha j}^{\mathrm{g} \beta}+C_{\beta j}^{\mathrm{g} \alpha}\right)}{2}=\left\langle C\left(\mathcal{P}_{V} v\right) v_{H}, \mathcal{P}_{V} v\right\rangle .
$$

Remark 3.2. We have

$$
-\mathcal{H}_{H}=\operatorname{div}_{H} v_{H}=\operatorname{div}_{H}\left(\frac{\mathcal{P}_{H} v}{\left|\mathcal{P}_{H} v\right|}\right)=\frac{\operatorname{div}_{H}\left(\mathcal{P}_{H} v\right)-\left\langle\operatorname{grad}_{H}\left|\mathcal{P}_{H} v\right|, v_{H}\right\rangle}{\left|\mathcal{P}_{H} v\right|}
$$

Since $\left|\mathcal{P}_{H} v\right|$ is Lipschitz continuous, it follows that $\mathcal{H}_{H} \in L_{\text {loc }}^{1}\left(S ; \sigma_{H}^{n-1}\right)$, but not necessarily $L_{\text {loc }}^{1}\left(S ; \sigma_{\mathcal{R}}^{n-1}\right)$. Note also that the last condition follows by assuming $\frac{1}{\left|\mathcal{P}_{H} v\right|} \in L_{\text {loc }}^{1}\left(S ; \sigma_{\mathcal{R}}^{n-1}\right)$.

Lemma 3.3. The following identities hold:
(i) $\phi_{1 i}\left(\tau_{j}\right)=\phi_{1 j}\left(\tau_{i}\right)+\left\langle C_{H}\left(\varpi_{H_{2}}\right) \tau_{i}, \tau_{j}\right\rangle \quad \forall i, j \in I_{H S}$;
(ii) $\phi_{1 i}\left(\tau_{\alpha}^{T S}\right)=\tau_{i}\left(\varpi_{\alpha}\right)+\frac{1}{2}\left\langle C_{H}^{\alpha} \tau_{1}, \tau_{i}\right\rangle-\left\langle C(\varpi) \tau_{\alpha}^{T S}, \tau_{i}\right\rangle \quad \forall i \in I_{H} \forall \alpha \in I_{V}$;
(iii) $\phi_{i \alpha}\left(\tau_{j}\right)=\phi_{j \alpha}\left(\tau_{i}\right)+\left\langle C_{H}^{\alpha} \tau_{i}, \tau_{j}\right\rangle \quad \forall i, j \in I_{H} \forall \alpha \in I_{V}$;
(iv) $\tau_{\alpha}^{T S}\left(\varpi_{\beta}\right)-\tau_{\beta}^{T S}\left(\varpi_{\alpha}\right)=\left\langle C(\varpi) \tau_{\beta}^{T S}, \tau_{\alpha}^{T S}\right\rangle \quad \forall \alpha, \beta \in I_{V}$;
(v) $\phi_{i \alpha}\left(\tau_{\alpha}\right)=0 \quad \forall i \in I_{H} \forall \alpha \in I_{V}$;
(vi) $\phi_{\alpha i}\left(\tau_{i}\right)=0 \quad \forall i \in I_{H} \forall \alpha \in I_{V}$;
(vii) $\phi_{i \alpha}\left(\tau_{j}\right)=\frac{1}{2}\left\langle C_{H}^{\alpha} \tau_{i}, \tau_{j}\right\rangle \quad \forall i, j \in I_{H} \forall \alpha \in I_{V}$.

Proof. By direct computation. In particular, using the fact that the Lie brackets of tangent vector fields along $S$ is still tangent; for a detailed proof, see [56].

Lemma 3.4. The matrix of the linear operator $B_{H}$ can be written out as a sum of two matrices, one symmetric and the other skew-symmetric, i.e. $B_{H}=S_{H}+A_{H}$, where the skew-symmetric matrix $A_{H}$ is given by $A_{H}=\left.\frac{1}{2} C_{H}\left(\varpi_{H_{2}}\right)\right|_{H S}$.

Proof. It is sufficient to apply (i) of Lemma 3.3

Lemma 3.5. One has $\operatorname{Tr}\left(B_{H}^{2}\right)=\left\|S_{H}\right\|_{\mathrm{G} r}^{2}-\left\|A_{H}\right\|_{\mathrm{G} r}^{2}=\sum_{j, k \in I_{H S}} \phi_{1 k}\left(\tau_{j}\right) \phi_{1 j}\left(\tau_{k}\right)$.

Proof. We have

$$
\begin{aligned}
\sum_{j, k \in I_{H S}} \phi_{1 k}\left(\tau_{j}\right) \phi_{1 j}\left(\tau_{k}\right) & =\sum_{j, k \in I_{H S}}\left\langle\nabla_{\tau_{j}} \tau_{1}, \tau_{k}\right\rangle\left\langle\nabla_{\tau_{k}} \tau_{1}, \tau_{j}\right\rangle \\
& =\sum_{j, k \in I_{H S}}\left(B_{H}\right)_{k j}\left(B_{H}\right)_{j k} \\
& =\operatorname{Tr}\left(B_{H}^{2}\right) \\
& =\sum_{j \in I_{H S}}\left\langle B_{H} \tau_{j}, B_{H}^{\operatorname{Tr}} \tau_{j}\right\rangle \\
& =\sum_{j \in I_{H S}}\left\langle\left(S_{H}+A_{H}\right) \tau_{j},\left(S_{H}-A_{H}\right) \tau_{j}\right\rangle \\
& =\left\|S_{H}\right\|_{\mathrm{G} r}^{2}-\left\|A_{H}\right\|_{\mathrm{G} r}^{2} .
\end{aligned}
$$

Lemma 3.6. We have $\sum_{\alpha \in I_{V}} \varpi_{\alpha} \mathcal{D}_{H S}\left(C_{H}^{\alpha} \tau_{1}\right)=2\left\|A_{H}\right\|_{\mathrm{G} r}^{2}+\left|C_{H}\left(\varpi_{H_{2}}\right) \tau_{1}\right|^{2}$. 
Proof. We have

$$
\begin{aligned}
\mathcal{D}_{H S}\left(C_{H}^{\alpha} \tau_{1}\right) & =\sum_{j \in I_{H S}}\left\langle\nabla_{\tau_{j}} C_{H}^{\alpha} \tau_{1}, \tau_{j}\right\rangle+\left\langle C_{H}^{\alpha} \tau_{1}, C_{H}\left(\varpi_{H_{2}}\right) \tau_{1}\right\rangle \\
& =-\sum_{j \in I_{H S}}\left\langle\nabla_{\tau_{j}} \tau_{1}, C_{H}^{\alpha} \tau_{j}\right\rangle+\left\langle C_{H}^{\alpha} \tau_{1}, C_{H}\left(\varpi_{H_{2}}\right) \tau_{1}\right\rangle \quad \text { (by linearity and skew-symmetry) } \\
& =-\sum_{j \in I_{H S}}\left\langle\nabla_{\tau_{j}} \tau_{1}, C_{H S}^{\alpha} \tau_{j}\right\rangle+\left\langle C_{H}^{\alpha} \tau_{1}, C_{H}\left(\varpi_{H_{2}}\right) \tau_{1}\right\rangle,
\end{aligned}
$$

where $C_{H S}^{\alpha}:=\left.C_{H}^{\alpha}\right|_{H S}$. Since $\left\langle\nabla_{\tau_{j}} \tau_{1}, C_{H S}^{\alpha} \tau_{j}\right\rangle=-B_{H}\left(\tau_{j}, C_{H S}^{\alpha} \tau_{j}\right) \forall j \in I_{H S}$, it follows that

$$
\begin{aligned}
\sum_{\alpha \in I_{V}} \varpi_{\alpha} \mathcal{D}_{H S}\left(C_{H}^{\alpha} \tau_{1}\right) & =\sum_{\alpha \in I_{V}} \varpi_{\alpha} \sum_{j \in I_{H S}} B_{H}\left(\tau_{j}, C_{H S}^{\alpha} \tau_{j}\right)+\left|C_{H}\left(\varpi_{H_{2}}\right) \tau_{1}\right|^{2} \\
& =\varpi_{\alpha} \sum_{j \in I_{H S}} B_{H}\left(\tau_{j}, C_{H S}\left(\varpi_{H_{2}}\right) \tau_{j}\right)+\left|C_{H}\left(\varpi_{H_{2}}\right) \tau_{1}\right|^{2},
\end{aligned}
$$

where $C_{H S}\left(\varpi_{H_{2}}\right)=\left.C_{H}\left(\varpi_{H_{2}}\right)\right|_{H S}=2 A_{H}$; see Lemma 3.4, Therefore

$$
\begin{aligned}
\sum_{\alpha \in I_{V}} \varpi_{\alpha} \mathcal{D}_{H S}\left(C_{H}^{\alpha} \tau_{1}\right) & =2 \sum_{j \in I_{H S}} B_{H}\left(\tau_{j}, A_{H} \tau_{j}\right)+\left|C_{H}\left(\varpi_{H_{2}}\right) \tau_{1}\right|^{2} \\
& =2 \sum_{j \in I_{H S}}\left\langle\left(S_{H}+A_{H}\right) \tau_{j}, A_{H} \tau_{j}\right\rangle+\left|C_{H}\left(\varpi_{H_{2}}\right) \tau_{1}\right|^{2} \\
& =2\left\|A_{H}\right\|_{\mathrm{G} r}^{2}+\left|C_{H}\left(\varpi_{H_{2}}\right) \tau_{1}\right|^{2},
\end{aligned}
$$

where we have used the elementary identity $\sum_{j \in I_{H S}}\left\langle S_{H} \tau_{j}, A_{H} \tau_{j}\right\rangle=0$. Let us prove the last identity. For every $j \in I_{H S}$ one has

$$
\begin{aligned}
\left\langle S_{H} \tau_{j}, A_{H} \tau_{j}\right\rangle & =\frac{1}{4}\left\langle\left(B_{H}+B_{H}^{\operatorname{Tr}}\right) \tau_{j},\left(B_{H}-B_{H}^{\operatorname{Tr}}\right) \tau_{j}\right\rangle \\
& =\frac{1}{4}\left(\left\langle B_{H} \tau_{j}, B_{H} \tau_{j}\right\rangle-\left\langle B_{H}^{\operatorname{Tr}} \tau_{j}, B_{H}^{\operatorname{Tr}} \tau_{j}\right\rangle\right) .
\end{aligned}
$$

By summing over $j \in I_{H S}$ we get that $\operatorname{Tr}\left(S_{H}\left(\cdot, A_{H} \cdot\right)\right)=\left\|B_{H}\right\|_{\mathrm{G} r}^{2}-\left\|B_{H}^{\mathrm{Tr}}\right\|_{\mathrm{G} r}^{2}=0$.

We now recall some identities involving the (Riemannian) curvature 2-forms $\Phi_{I J}$ associated with the o.n. co-frame $\underline{\phi}$ (dual of $\underline{\tau}$ ) which can be found in [56]. In particular, we need to calculate $\sum_{j \in I_{H S}} \Phi_{1 j}\left(X, \tau_{j}\right)=$ $\sum_{j \in I_{H S}}\left\langle\mathrm{R}\left(X, \bar{\tau}_{j}\right) \tau_{1}, \tau_{j}\right\rangle$ for any $X \in v_{H} S$, which is nothing but the Ricci curvature for the partial $H S$ connection $\nabla^{H S}$.

Lemma 3.7. We have:

(i) $\left\langle\mathrm{R}\left(\tau_{i}, \tau_{j}\right) \tau_{h}, \tau_{k}\right\rangle=-\frac{3}{4} \sum_{\alpha \in I_{H_{2}}}\left\langle C_{H}^{\alpha} \tau_{i}, \tau_{j}\right\rangle\left\langle C_{H}^{\alpha} \tau_{h}, \tau_{k}\right\rangle \quad \forall i j, h, k \in I_{H}$;

(ii) $\left\langle\mathrm{R}\left(\tau_{\beta}, \tau_{i}\right) \tau_{j}, \tau_{k}\right\rangle=-\frac{1}{4} \sum_{\alpha \in I_{H_{2}}}\left\langle C_{H}^{\alpha} \tau_{j}, \tau_{k}\right\rangle\left\langle C^{\beta} \tau_{\alpha}, \tau_{i}\right\rangle \quad \forall i, j, k \in I_{H}, \beta \in I_{H_{3}}$.

Lemma 3.8. For every $X=X_{H}+X_{V} \in \mathfrak{X}(\mathbb{G})$ transversal to $S$, i.e. $X \pitchfork S$, we have the formula

$$
\sum_{j \in I_{H S}} \Phi_{1 j}\left(X, \tau_{j}\right)=-\frac{3}{4} \sum_{\alpha \in I_{H_{2}}}\left\langle C_{H}^{\alpha} v_{H}, C_{H}^{\alpha} X_{H}\right\rangle-\frac{1}{4} \sum_{\alpha \in I_{H_{2}}} \sum_{\beta \in I_{H_{3}}} x_{\beta}\left\langle C_{H}^{\alpha} v_{H}, C^{\beta} \tau_{\alpha}\right\rangle .
$$

Proof. Using Lemma 3.7

Lemma 3.9. Let $\underline{\tau}=\left\{\tau_{1}, \ldots, \tau_{n}\right\}$ be an adapted o.n. frame for $\mathcal{U} \subseteq S$ on $U$ and fix $p_{0} \in \mathcal{U}$. Then, we can always choose $\underline{\tau}$ so that the connection 1-forms $\underline{\phi}=\left\{\phi_{1}, \ldots, \phi_{n}\right\}$ satisfy $\phi_{i j}\left(p_{0}\right)=0$ whenever $i, j \in I_{H S}=\{2, \ldots, h\}$. 
Proof. The proof follows by using a Riemannian geodesic frame. So let $\xi=\left\{\xi_{1}, \ldots, \xi_{n}\right\}$ be a o.n. frame on $U$ adapted to $\mathcal{U}=U \cap S$ satisfying $\xi_{1}(p)=v(p)$ and such that $T_{p} S=\operatorname{span}_{\mathbb{R}}\left\{\xi_{2}(p), \ldots, \xi_{n}(p)\right\}$ for every $p \in \mathcal{U}$. Let $\underline{\varepsilon}=\left\{\varepsilon_{1}, \ldots, \varepsilon_{n}\right\}$ denote its dual co-frame.

Claim 3.10. It is always possible to choose another o.n. frame $\underline{\xi}$ on $U$ adapted to $\mathcal{U}$ satisfying:

(i) $\underline{\xi}\left(p_{0}\right)=\underline{\xi}\left(p_{0}\right)$;

(ii) Let $\widetilde{\varepsilon}_{i j}:=\left\langle\nabla \widetilde{\xi}_{i}, \widetilde{\xi}_{j}\right\rangle(i, j=1, \ldots, n)$ denote the connection 1-forms of $\underline{\xi}$. Then, one has $\widetilde{\varepsilon}_{i j}\left(p_{0}\right)=0$ for every $i, j=2, \ldots, n$.

Clearly $\underline{\xi}=\left\{\widetilde{\xi}_{2}, \ldots, \widetilde{\xi}_{n}\right\}$ is a tangent o.n. frame for $\mathcal{U}$. The proof of this claim is standard; see, for instance, [72], pag. 517-519, eq.(17). Now assuming that $\xi_{i}\left(p_{0}\right)=\tau_{i}\left(p_{0}\right)$ for every $i \in I_{H S}$. In particular, we have

$$
\widetilde{\varepsilon}_{i j}\left(X_{p_{0}}\right)=\left\langle\nabla_{X_{p_{0}}} \widetilde{\xi}_{i}, \widetilde{\xi}_{j}\right\rangle\left(p_{0}\right)=0 \quad \forall i, j \in I_{H S}, \forall X \in \mathfrak{X}^{1}(T S) .
$$

By extending the o.n. frame $\left\{\widetilde{\xi}_{2}, \ldots, \widetilde{\xi}_{h}\right\}$ for the horizontal tangent space $H S$ to a full adapted frame $\underline{\tau}$ in the sense of Definition 1.9, the thesis easily follows.

The following notion will be used throughout the proof of Lemma 5.5 .

Definition 3.11. Let $S \subset \mathbb{G}$ be a hypersurface of class $\mathbf{C}^{i}(i \geq 2)$. We say that a $\mathbf{C}^{i}$-smooth function $f: \mathbb{G} \longrightarrow \mathbb{R}$ is a defining function for $S$ if $S=\{x \in \mathbb{G}: f=0\}$ and gradf $\neq 0$ for all $x \in S$. Furthermore, we say that $f$ is a normalized defining function for $S$ (abbreviated as NDF) if, and only if, $\left|\operatorname{grad}_{H} f\right|=1$ for all $x \in S \backslash C_{S}$.

Remark 3.12. Some remarks are in order. First, it is not difficult to see that, given a defining function $f$ for $S$, then a NDF $\widetilde{f}$ for $S$ can simply be defined by dividing $f$ by the magnitude of its horizontal gradient $\mid \operatorname{grad}_{H} f$, i.e.

$$
\operatorname{grad} \widetilde{f}(p)=\operatorname{grad}\left(\frac{f}{\left|\operatorname{grad}_{H} f\right|}\right)(p)=\frac{\operatorname{grad} f}{\left|\operatorname{grad}_{H} f\right|}(p)=v_{H}(p)+\varpi(p) \quad \forall p \in S \backslash C_{S} .
$$

Note that the NDF $\widetilde{f}$ is one order of differentiability less smooth than $f$. This is what happens also in the Euclidean case; see the book by Krantz and Parks [46] and references therein. However, at least for 2-step Carnot groups, a normalized defining function of class $\mathbf{C}^{i}$ for every $\mathbf{C}^{i}$-smooth hypersurface $S$ $(i \geq 2)$, is given by the (signed) CC-distance function from $S$; see [8].

We end this section with a lemma, which will be important in the sequel. Let $S$ be as above, let $p_{0} \in S$ and assume that, locally around $p_{0}, S$ is the level set of a function $f: U \subset \mathbb{G} \longrightarrow \mathbb{R}$. We see that, locally around $p_{0}, X f=0$ for every $X \in \mathfrak{X}(T S)$. In particular, $\tau_{\alpha}^{T S}(f)=0$ for every $\alpha \in I_{V}$. As a consequence, by using an adapted frame $\underline{\tau}$, one has $\tau_{\alpha}(f)=\varpi_{\alpha} \tau_{1}(f)$ for every $\alpha \in I_{V}$. A normal vector along $S$ in a neighborhood of $p_{0}$ is given by $\mathcal{N}:=\tau_{1}(f) \tau_{1}+\sum_{\alpha \in I_{V}} \tau_{\alpha}(f) \tau_{\alpha}$ and we have $v=\frac{\mathcal{N}}{|\mathcal{N}|}$.

Lemma 3.13. The following identities hold:

(i) $\phi_{1 j}\left(\tau_{1}\right)=\frac{\tau_{j}\left(\tau_{1}(f)\right)}{\tau_{1}(f)}-\left\langle C_{H}\left(\varpi_{H_{2}}\right) \tau_{1}, \tau_{j}\right\rangle \quad \forall j \in I_{H S}$;

(ii) $\phi_{1 j}\left(\tau_{\alpha}\right)=\frac{1}{2}\left\langle C_{H}^{\alpha} \tau_{1}, \tau_{j}\right\rangle-\left\langle C(\varpi) \tau_{\alpha}, \tau_{j}\right\rangle+\frac{\tau_{j}\left(\tau_{\alpha}(f)\right)}{\tau_{1}(f)} \quad \forall j \in I_{H S} \forall \alpha \in I_{V}$.

Proof. We have

$$
\left[\tau_{1}, \tau_{j}\right]=\left\langle\left[\tau_{1}, \tau_{j}\right], \tau_{1}\right\rangle \tau_{1}+\sum_{k \in I_{H S}}\left\langle\left[\tau_{1}, \tau_{j}\right], \tau_{k}\right\rangle \tau_{k}+\sum_{\alpha \in I_{V}}\left\langle\left[\tau_{1}, \tau_{j}\right], \tau_{\alpha}\right\rangle \tau_{\alpha}
$$

Therefore

$$
\left[\tau_{1}, \tau_{j}\right](f)=-\tau_{j}\left(\tau_{1}(f)\right)=\left\langle\left[\tau_{1}, \tau_{j}\right], \tau_{1}\right\rangle \tau_{1}(f)+\sum_{\alpha \in I_{V}}\left\langle\left[\tau_{1}, \tau_{j}\right], \tau_{\alpha}\right\rangle \tau_{\alpha}(f)
$$


and this implies that

$$
C_{1 j}^{1}=\phi_{1 j}\left(\tau_{1}\right)=\left\langle\left[\tau_{1}, \tau_{j}\right], \tau_{1}\right\rangle=\frac{\tau_{j}\left(\tau_{1}(f)\right)}{\tau_{1}(f)}-\sum_{\alpha \in I_{V}} \frac{\tau_{\alpha}(f)}{\tau_{1}(f)}\left\langle C_{H}^{\alpha} \tau_{1}, \tau_{j}\right\rangle,
$$

where we have used the identity $C_{1 j}^{\alpha}=-\left\langle C_{H}^{\alpha} \tau_{1}, \tau_{j}\right\rangle$. This proves (i).

In order to prove (ii), we compute

$$
\left[\tau_{\alpha}, \tau_{j}\right]=\left\langle\left[\tau_{\alpha}, \tau_{j}\right], \tau_{1}\right\rangle \tau_{1}+\sum_{k \in I_{H S}}\left\langle\left[\tau_{\alpha}, \tau_{j}\right], \tau_{k}\right\rangle \tau_{k}+\sum_{\beta \in I_{V}}\left\langle\left[\tau_{\alpha}, \tau_{j}\right], \tau_{\beta}\right\rangle \tau_{\beta},
$$

from which we get

$$
\left[\tau_{\alpha}, \tau_{j}\right](f)=-\tau_{j}\left(\tau_{\alpha}(f)\right)=\left\langle\left[\tau_{\alpha}, \tau_{j}\right], \tau_{1}\right\rangle \tau_{1}(f)+\sum_{\beta \in I_{V}}\left\langle\left[\tau_{\alpha}, \tau_{j}\right], \tau_{\beta}\right\rangle \tau_{\beta}(f) .
$$

Thus

$$
-\frac{\tau_{j}\left(\tau_{\alpha}(f)\right)}{\tau_{1}(f)}=-\phi_{1 j}\left(\tau_{\alpha}\right)+\phi_{1 \alpha}\left(\tau_{j}\right)+\sum_{\beta \in I_{V}} \varpi_{\beta} C_{\alpha j}^{1},
$$

where we have used the identity $C_{\alpha j}^{1}=\left\langle\nabla_{\tau_{\alpha}} \tau_{j}, \tau_{1}\right\rangle-\left\langle\nabla_{\tau_{j}} \tau_{\alpha}, \tau_{1}\right\rangle$. Finally, since $\phi_{1 \alpha}\left(\tau_{j}\right)=\frac{1}{2}\left\langle C_{H}^{\alpha} \tau_{1}, \tau_{j}\right\rangle$ (see (vii) of Lemma 3.3), using $C_{\alpha j}^{\beta}=-\left\langle C^{\beta} \tau_{\alpha}, \tau_{j}\right\rangle$ it follows that

$$
\phi_{1 j}\left(\tau_{\alpha}\right)=\frac{1}{2}\left\langle C_{H}^{\alpha} \tau_{1}, \tau_{j}\right\rangle-\sum_{\beta \in I_{V}} \varpi_{\beta}\left\langle C^{\beta} \tau_{\alpha}, \tau_{j}\right\rangle+\frac{\tau_{j}\left(\tau_{\alpha}(f)\right)}{\tau_{1}(f)},
$$

as wished.

\section{VARIATIONAL Formulae For the $H$-PeRIMETER $\sigma_{H}^{n-1}$}

Below we will obtain the 1 st and 2 nd variation formulae for the $H$-perimeter measure $\sigma_{H}^{n-1}$. More precisely, we shall assume that $S \subset \mathbb{G}$ is of class $\mathbf{C}^{2}$, for the 1 st variation formula, and that $S$ is of class $\mathbf{C}^{3}$ for the 2 nd variation formula. Under further hypotheses, our formulae allow to move the characteristic set $C_{S}$ of $S$.

We stress that, in the case of the first Heisenberg group $\mathbb{H}^{1}$, a 1 st variation formula for characteristic surfaces of class $\mathbf{C}^{2}$ was obtained by Ritoré and Rosales in [67]. Furthermore, Hurtado, Ritoré and Rosales [44] have proved a formula for the 2nd variation of $\sigma_{H}^{n-1}$ that is very similar to that stated in Theorem 4.12 below; see also the unpublished preprint [43], where similar results are stated in a general sub-Riemannian setting.

Let $S \subset \mathbb{G}$ be a hypersurface of class $\mathbf{C}^{i}(i=2,3)$, let $U \subset \mathbb{G}$ be a relatively compact open set having non-empty intersection with $S$ and set $\mathcal{U}:=U \cap S$.

The following calculations are made for a bounded open subset $\mathcal{U}$ of $S$. In particular, we assume $\mathbf{C}^{1}$-regularity of $\partial \mathcal{U}$. Clearly, if $S$ is a compact hypersurface with boundary, the formulae obtained in the sequel will hold for $S$.

Definition 4.1. Let $\imath: \mathcal{U} \rightarrow \mathbb{G}$ denote the inclusion of $\mathcal{U} \subset S$ in $\mathbb{G}$ and let $\vartheta:]-\epsilon, \epsilon[\times \mathcal{U} \rightarrow \mathbb{G}$ be a $\mathbf{C}^{i}$-smooth map, $i=2,3$. We say that $\vartheta$ is a variation of $\iota$ if:

(i) every $\vartheta_{t}:=\vartheta(t, \cdot): \mathcal{U} \rightarrow \mathbb{G}$ is an immersion;

(ii) $\vartheta_{0}=\imath$.

Moreover, we say that $\vartheta$ keeps the boundary $\partial \mathcal{U}$ fixed if:

(iii) $\left.\vartheta_{t}\right|_{\partial \mathcal{U}}=\left.\imath\right|_{\partial \mathcal{U}}$ for every $\left.t \in\right]-\epsilon, \epsilon[$.

The variation vector of $\vartheta$ (i.e. its “initial velocity") is defined by $W:=\left.\frac{\partial \vartheta}{\partial t}\right|_{t=0}=\left.\vartheta * \frac{\partial}{\partial t}\right|_{t=0}$. 
We shall set $\widetilde{W}:=\frac{\partial \vartheta}{\partial t}=\vartheta_{*} \frac{\partial}{\partial t}$ and assume that $\widetilde{W}$ is defined in a neighborhood of $\operatorname{Im}(\vartheta)$. For any "time" $t \in]-\epsilon, \epsilon$, let $v^{t}$ be the unit normal vector along $\mathcal{U}_{t}:=\vartheta_{t}(\mathcal{U})$ and let $\left(\sigma_{\mathcal{R}}^{n-1}\right)_{t}$ be the Riemannian measure on $\mathcal{U}_{t}$. We assume that $f: U \longrightarrow \mathbb{R}$ is a local equation for the hypersurface $S$ near $p_{0} \in S$ and that $\left.f_{t}:\right]-\epsilon, \epsilon\left[\times U \longrightarrow \mathbb{R}\right.$ is a family of $\mathbf{C}^{i}$-smooth functions $(i=2,3)$ satisfying $f_{0}=f$ and $f_{t}\left(\vartheta_{t}(x)\right)=t$ for every $t \in]-\epsilon, \epsilon$. In other words, the hypersurfaces $\mathcal{U}_{t}$ are level sets of a defining function $f_{t}$ and one has $\left\langle\nabla f_{t}, \widetilde{W}\right\rangle=1$. Choose an o.n. frame $\underline{\tau}$ on $U \subset \mathbb{G}$ satisfying:

$$
\tau_{1} \mid \mathcal{U}_{t}=v_{H}^{t} ; \quad H T_{p} \mathcal{U}_{t}=\operatorname{span}\left\{\left(\tau_{2}\right)_{p}, \ldots,\left(\tau_{h}\right)_{p}\right\} \quad \forall p \in \mathcal{U}_{t} ; \quad \tau_{\alpha}=X_{\alpha}
$$

for every $t \in]-\epsilon, \epsilon$. Furthermore, let $\underline{\phi}:=\left\{\phi_{1}, \ldots, \phi_{n}\right\}$ be the dual co-frame of $\underline{\tau}$ (i.e. $\phi_{i}\left(\tau_{j}\right)=\delta_{i}^{j}$ for all $i, j=1, \ldots, n)$. So, we have $\tau_{\alpha}^{T S} f_{t}=0$; see Definition 1.9. This implies $\tau_{\alpha}\left(f_{t}\right)=\varpi_{\alpha}^{t} \tau_{1}\left(f_{t}\right)$, where $\varpi_{\alpha}^{t}:=\frac{v_{\alpha}^{t}}{\left|\mathcal{P}_{H} v^{t}\right|}$. Moreover, since $\left\langle\nabla f_{t}, \widetilde{W}\right\rangle=1$, we have $\widetilde{w}_{1} \tau_{1}\left(f_{t}\right)+\sum_{\alpha \in I_{V}} \widetilde{w}_{\alpha} \tau_{\alpha}\left(f_{t}\right)=1$, where $\widetilde{w}_{1}=\left\langle\widetilde{W}, \tau_{1}\right\rangle$ and $\widetilde{w}_{\alpha}=\left\langle\widetilde{W}, \tau_{\alpha}\right\rangle$. Therefore

$$
\tau_{1}\left(f_{t}\right)\left(\widetilde{w}_{1}+\sum_{\alpha \in I_{V}} \widetilde{w}_{\alpha} \varpi_{\alpha}^{t}\right)=1
$$

Setting $w_{t}=\frac{\left\langle\widetilde{W}, v^{t}\right\rangle}{\left|\mathcal{P}_{H} v^{t}\right|}$ it follows that $\tau_{1}\left(f_{t}\right)=\frac{1}{w_{t}}$ and $\tau_{\alpha}\left(f_{t}\right)=\frac{\varpi_{\alpha}^{t}}{w_{t}}$.

The following technical result will be used in the proof of the 2nd variation of $\sigma_{H}^{n-1}$.

Lemma 4.2. Under the previous assumptions, we have:

(i) $\mathcal{P}_{H S_{t}}\left(\nabla_{\tau_{1}} \tau_{1}\right)=-\left(\frac{\operatorname{grad}_{H S_{t}} w_{t}}{w_{t}}+C_{H}\left(\varpi_{H_{2}}^{t}\right) \tau_{1}\right)$;

(ii) $\mathcal{P}_{H S_{t}}\left(\nabla_{\tau_{\alpha}} \tau_{1}\right)=\frac{1}{2} C_{H}^{\alpha} \tau_{1}-C\left(\varpi^{t}\right) \tau_{\alpha}+\operatorname{grad}_{H S_{t}} \varpi_{\alpha}^{t}-\varpi_{\alpha}^{t} \frac{\operatorname{grad}_{H S_{t}} w_{t}}{w_{t}} \quad \forall \alpha \in I_{V}$.

Proof. By applying (i) of Lemma 3.13 we get that $\phi_{1 j}\left(\tau_{1}\right)=-\frac{\tau_{j}\left(w_{t}\right)}{w_{t}}-\left\langle C_{H}\left(\varpi_{H_{2}}^{t}\right) \tau_{1}, \tau_{j}\right\rangle$. Furthermore, (ii) of Lemma 3.13 implies

$$
\phi_{1 j}\left(\tau_{\alpha}\right)=\frac{1}{2}\left\langle C_{H}^{\alpha} \tau_{1}, \tau_{j}\right\rangle-\left\langle C\left(\varpi^{t}\right) \tau_{\alpha}, \tau_{j}\right\rangle+\tau_{j}\left(\varpi_{\alpha}^{t}\right)-\varpi_{\alpha}^{t} \frac{\tau_{j}\left(w_{t}\right)}{w_{t}} \quad \forall \alpha \in I_{V}
$$

This achieves the proof.

General remarks. In order to discuss the variational formulae of $\sigma_{H}^{n-1}$, let us set

$$
\left.\left(\sigma_{H}^{n-1}\right)_{t}\left\llcorner\mathcal{U}_{t}=\left(\tau_{1}\right\lrcorner \phi_{1} \wedge \ldots \wedge \phi_{n}\right)\right|_{\mathcal{U}_{t}}=\left.\left(\phi_{2} \wedge \ldots \wedge \phi_{n}\right)\right|_{\mathcal{U}_{t}} .
$$

We also set $\Gamma(t):=\vartheta_{t}^{*}\left(\phi_{2} \wedge \ldots \wedge \phi_{n}\right)$. Note that $\left.\Gamma:\right]-\epsilon, \epsilon\left[\times \mathcal{U} \longrightarrow \Omega^{n-1}(\mathcal{U})\right.$ defines a 1-parameter family of differential $(n-1)$-forms on $\mathcal{U}$.

Remark 4.3. By definition, the 1 st and 2 nd variation formulae of $\sigma_{H}^{n-1}$ along $\mathcal{U}$ are given by

$$
I_{\mathcal{U}}\left(\sigma_{H}^{n-1}\right):=\left.\frac{d}{d t}\left(\int_{\mathcal{U}} \Gamma(t)\right)\right|_{t=0}, \quad I I_{\mathcal{U}}\left(\sigma_{H}^{n-1}\right):=\left.\frac{d^{2}}{d t^{2}}\left(\int_{\mathcal{U}} \Gamma(t)\right)\right|_{t=0} .
$$

So we have a natural question: is it possible to bring the "time" derivatives inside the integral sign? Note that the answer is "yes" if we assume that $\overline{\mathcal{U}}$ is non-characteristic. Indeed, in such a case it is not difficul $\square^{7}$ to show that there exists $\epsilon>0$ such that the 1-parameter family $\Gamma(\cdot)$ of differential $(n-1)$-forms on $\mathcal{U}$ is $\mathbf{C}^{i-1}$-smooth on $]-\epsilon, \epsilon[\times \mathcal{U}$. This allows us to estimate, uniformly in time, both differential $(n-1)$-forms $\dot{\Gamma}(t)$ and $\ddot{\Gamma}(t)$. We will return on this point later in this section.

\footnotetext{
${ }^{7}$ Actually, since $\operatorname{grad}_{H} f_{t} \neq 0$ at $t=0$, there must exist $\epsilon>0$ such that $\operatorname{grad}_{H} f_{t} \neq 0$ for all $\left.t \in\right]-\epsilon, \epsilon\left[\right.$ and hence $v_{H}^{t}=\frac{\operatorname{grad}_{H} f_{t}}{\left|\operatorname{grad}_{H} f_{t}\right|}$, which is the unit $H$-normal along $\mathcal{U}_{t}=\vartheta_{t}(\mathcal{U})$, turns out to be of class $\mathbf{C}^{i-1}, i=2,3$. This implies that $\left(\sigma_{H}^{n-1}\right)_{t}$ is $\mathbf{C}^{i-1}$-smooth. Therefore $\Gamma(t)=\vartheta_{t}^{*}\left(\sigma_{H}^{n-1}\right)_{t}$ is $\mathbf{C}^{i-1}$-smooth.
} 
Warning 4.4. Preliminarily, we need the following assumptions:

$\left(A_{1}\right)$ if $\mathcal{U}$ is of class $\mathbf{C}^{2}$ there exists an integrable differential $(n-1)$-form $\Phi_{1} \in \Omega^{n-1}(\mathcal{U})$, such that:

$$
\left|\dot{\Gamma}(t)\left(\mathrm{t}_{1}, \ldots, \mathrm{t}_{n-1}\right)\right| \leq\left|\Phi_{1}\left(\mathrm{t}_{1}, \ldots, \mathrm{t}_{n-1}\right)\right|
$$

for every o.n. basis $\underline{\mathrm{t}}=\left\{\mathrm{t}_{1}, \ldots, \mathrm{t}_{n-1}\right\}$ of $T \mathcal{U}$.

$\left(A_{2}\right)$ if $\mathcal{U}$ is of class $\mathbf{C}^{3}$ there exist integrable differential $(n-1)$-forms $\Phi_{1}, \Phi_{2} \in \Omega^{n-1}(\mathcal{U})$, such that:

$$
\begin{aligned}
&\left|\dot{\Gamma}(t)\left(\mathrm{t}_{1}, \ldots, \mathrm{t}_{n-1}\right)\right| \leq\left|\Phi_{1}\left(\mathrm{t}_{1}, \ldots, \mathrm{t}_{n-1}\right)\right| \\
&\left|\ddot{\Gamma}(t)\left(\mathrm{t}_{1}, \ldots, \mathrm{t}_{n-1}\right)\right| \leq\left|\Phi_{2}\left(\mathrm{t}_{1}, \ldots, \mathrm{t}_{n-1}\right)\right|
\end{aligned}
$$

for every o.n. basis $\underline{\mathrm{t}}=\left\{\mathrm{t}_{1}, \ldots, \mathrm{t}_{n-1}\right\}$ of $T \mathcal{U}$.

1st variation. We first note that

$$
\int_{\mathcal{U}} \Gamma(t)=\int_{\mathcal{U}} \vartheta_{t}^{*}\left(\sigma_{H}^{n-1}\right)_{t}=\int_{\mathcal{U}}\left|\mathcal{P}_{H_{t}} v^{t}\right| \mathcal{J} a c \vartheta_{t} \sigma_{\mathcal{R}}^{n-1},
$$

where $\mathcal{J} a c \vartheta_{t}$ denotes the usual Jacobian of the map $\vartheta_{t}$; see [70], Ch. 2, § 8, pp. 46-48. Indeed, by definition, we have $\left(\sigma_{H}^{n-1}\right)_{t}=\left|\mathcal{P}_{H_{t}} v^{t}\right|\left(\sigma_{\mathcal{R}}^{n-1}\right)_{t}$ and hence the previous formula follows from the Area formula of Federer; see [29] or [70]. Let us set $f:]-\epsilon, \epsilon[\times \mathcal{U} \longrightarrow \mathbb{R}$,

$$
f(t, x):=\left|\mathcal{P}_{H_{t}} v^{t}(x)\right| \mathcal{J} a c \vartheta_{t}(x) .
$$

In this case, we also set $C_{\mathcal{U}}:=\left\{x \in \mathcal{U}:\left|\mathcal{P}_{H_{t}} v^{t}(x)\right|=0\right\}$. With this notation, our original question can be solved by applying to $f$ the Theorem of Differentiation under the integral; see [45], Corollary 1.2.2, p.124. More precisely, let us compute

$$
\begin{aligned}
\frac{d f}{d t} & =\frac{d\left|\mathcal{P}_{H_{t}} v^{t}\right|}{d t} \mathcal{J} a c \vartheta_{t}+\left|\mathcal{P}_{H_{t}} v^{t}\right| \frac{d \mathcal{J} a c \vartheta_{t}}{d t} \\
& =\left\langle\widetilde{W}, \operatorname{grad}\left|\mathcal{P}_{H_{t}} v^{t}\right|\right\rangle \mathcal{J} a c \vartheta_{t}+\left|\mathcal{P}_{H_{t}} v^{t}\right| \frac{d \mathcal{J} a c \vartheta_{t}}{d t} \\
& =\left(\left\langle\widetilde{W}^{\perp}, \operatorname{grad}\left|\mathcal{P}_{H_{t}} v^{t}\right|\right\rangle+\left\langle\widetilde{W}^{\top}, \operatorname{grad}\left|\mathcal{P}_{H_{t}} v^{t}\right|\right\rangle+\left|\mathcal{P}_{H_{t}} v^{t}\right| \operatorname{div} v_{T} \mathcal{U}_{t} \widetilde{W}\right) \mathcal{J} a c \vartheta_{t} \\
& =\left(\left\langle\widetilde{W}^{\perp}, \operatorname{grad}\left|\mathcal{P}_{H_{t}} v^{t}\right|\right\rangle+\operatorname{div}_{T} \mathcal{U}_{t}\left(\widetilde{W}\left|\mathcal{P}_{H_{t}} v^{t}\right|\right)\right) \mathcal{J} a c \vartheta_{t},
\end{aligned}
$$

where we have used the very definition of tangential divergence and the well-known calculation of $\frac{d \mathcal{J} a c \vartheta_{t}}{d t}$, which can be found in Chavel's book [16]; see Ch.2, p.34. Now since $\left|\mathcal{P}_{H_{t}} v^{t}\right|$ is a Lipschitz continuous function, it follows that $\frac{d f}{d t}$ is bounded on $\mathcal{U} \backslash C_{\mathcal{U}}$ and so lies in $L_{l o c}^{1}\left(\mathcal{U} ; \sigma_{\mathcal{R}}^{n-1}\right)$. Therefore, we can pass the time-derivative through the integral sign. This shows that: condition $\left(A_{1}\right)$ in Warning 4.4 is always satisfied. In particular, we have proved the following 1st variation formula:

$$
I \mathcal{U}\left(\sigma_{H}^{n-1}\right)=\int_{\mathcal{U}} \dot{\Gamma}(0)=\int_{\mathcal{U}}\left(\left\langle W^{\perp}, \operatorname{grad}\left|\mathcal{P}_{H} v_{H}\right|\right\rangle+\operatorname{div}_{T \mathcal{U}}\left(W\left|\mathcal{P}_{H} v_{H}\right|\right)\right) \sigma_{\mathcal{R}}^{n-1}
$$

It follows from definitions that $\frac{d f}{d t}$ can be regarded as the Lie derivative of $\left(\sigma_{H}^{n-1}\right)_{t}$ with respect to the variation vector $\widetilde{W}$, that is

$$
\frac{d f}{d t}=\vartheta_{t}^{*} \mathcal{L}_{\widetilde{W}}\left(\sigma_{H}^{n-1}\right)_{t}
$$

Remark 4.5. Note that formula (20) can be proved exactly as in Spivak's book [72], Ch. 9, p. 420. As already mentioned in Section 2.1] this fact allows us to use some standard tools in Differential Geometry such as the Cartan's formula. In this way, another expression for the integrand $\dot{\Gamma}(0)$ can be derived; see, for instance, formula (8). Nevertheless, this new expression it is not necessarily in $L_{l o c}^{1}$, with respect to the Riemannian measure $\sigma_{\mathcal{R}}^{n-1}$; see Remark 2.15 
More precisely, we have

$$
\dot{\Gamma}(0)=\imath^{*}\left(\mathcal{L}_{\widetilde{W}}\left(\sigma_{H}^{n-1}\right)_{t}\right)=\imath^{*}\left(\mathcal{L}_{\widetilde{W}}\left(\phi_{2} \wedge \ldots \wedge \phi_{n}\right)\right)
$$

By Cartan's formula

$$
\left.\left.\mathcal{L}_{\widetilde{W}}\left(\sigma_{H}^{n-1}\right)_{t}=\widetilde{W}\right\lrcorner d\left(\sigma_{H}^{n-1}\right)_{t}+d(\widetilde{W}\lrcorner\left(\sigma_{H}^{n-1}\right)_{t}\right)
$$

and hence

$$
\left.\left.\dot{\Gamma}(0)=\imath^{*}(\widetilde{W}\lrcorner d\left(\sigma_{H}^{n-1}\right)_{t}+d(\widetilde{W}\lrcorner\left(\sigma_{H}^{n-1}\right)_{t}\right)\right) .
$$

By applying the 1st structure equation of the co-frame $\underline{\phi}$ (see formula (3) we have

$$
d\left(\sigma_{H}^{n-1}\right)_{t}=\sum_{j=2}^{n}(-1)^{j} \phi_{2} \wedge \ldots \wedge d \phi_{j} \wedge \ldots \wedge \phi_{n}=\sum_{j \in I_{H S}} \phi_{1 j}\left(\tau_{j}\right) \phi_{1} \wedge \ldots \wedge \phi_{n}=-\left(\mathcal{H}_{H}\right)_{t}\left(\sigma_{\mathcal{R}}^{n}\right)_{t},
$$

where $\left(\mathcal{H}_{H}\right)_{t}:=-\sum_{j \in I_{H S}} \phi_{1 j}\left(\tau_{j}\right)=\sum_{j \in I_{H S}}\left\langle\nabla_{\tau_{j}}^{H} \tau_{j}, v_{H}^{t}\right\rangle$ is the horizontal mean curvature of $\mathcal{U}_{t}$. Note that we have used (v) of Lemma 3.3 .

The calculation of the second term has been discussed in detail in Section 2, see Lemma 2.13, More precisely, we have

$$
\left.d(\widetilde{W}\lrcorner\left(\sigma_{H}^{n-1}\right)_{t}\right)=\operatorname{div}_{T} \mathcal{U}_{t}\left(\widetilde{W}^{\top}\left|\mathcal{P}_{H_{t}} v^{t}\right|-\left\langle\widetilde{W}, v^{t}\right\rangle v_{H}^{t}{ }^{\top}\right)\left(\sigma_{\mathcal{R}}^{n-1}\right)_{t} .
$$

Therefore, under the previous assumptions, we have

$$
\mathcal{L}_{\widetilde{W}}\left(\sigma_{H}^{n-1}\right)_{t}=\left(-\left(\mathcal{H}_{H}\right)_{t}\left\langle\widetilde{W}, v^{t}\right\rangle+\operatorname{div}_{T} \mathcal{U}_{t}\left(\widetilde{W}^{\top}\left|\mathcal{P}_{H_{t}} v^{t}\right|-\left\langle\widetilde{W}, v^{t}\right\rangle v_{H}^{t}{ }^{\top}\right)\right)\left(\sigma_{\mathcal{R}}^{n-1}\right)_{t}
$$

Finally, the desired formula follows by setting $t=0$; see formula (8).

Theorem 4.6 (1st variation of $\sigma_{H}^{n-1}$ ). Let $S \subset \mathbb{G}$ be a compact hypersurface of class $\mathbf{C}^{2}$ with, or without, boundary and let $\vartheta:]-\epsilon, \epsilon\left[\times S \rightarrow \mathbb{G}\right.$ be a $\mathbf{C}^{2}$-smooth variation of $S$. Let $W=\left.\frac{d \vartheta_{t}}{d t}\right|_{t=0}$ be the variation vector field and let $W^{\perp}$ and $W^{\top}$ be the normal and tangential components of $W$ along $S$, respectively. We also set $w:=\frac{\left\langle W^{\perp}, v\right\rangle}{\left|\mathcal{P}_{H} v\right|}$. Then

$$
I_{S}\left(\sigma_{H}^{n-1}\right)=\int_{S}\left(\left\langle W^{\perp}, \operatorname{grad}\left|\mathcal{P}_{H} v_{H}\right|\right\rangle+\operatorname{div}_{T S}\left(W\left|\mathcal{P}_{H} v_{H}\right|\right)\right) \sigma_{\mathcal{R}}^{n-1} .
$$

Furthermore, if $\mathcal{H}_{H} \in L^{1}\left(S ; \sigma_{\mathcal{R}}^{n-1}\right)$, then

$$
\begin{aligned}
I_{S}\left(W, \sigma_{H}^{n-1}\right) & =\int_{S}-\mathcal{H}_{H} w \sigma_{H}^{n-1}+\int_{S} \operatorname{div}_{T S}\left(W^{\top}\left|\mathcal{P}_{H} v\right|-\langle W, v\rangle v_{H}^{\top}\right) \sigma_{\mathcal{R}}^{n-1} \\
& =\int_{S}\left(-\mathcal{H}_{H}\left\langle W^{\perp}, v\right\rangle+\operatorname{div}_{T S}\left(W^{\top}\left|\mathcal{P}_{H} v\right|-\left\langle W^{\perp}, v\right\rangle v_{H}^{\top}\right)\right) \sigma_{\mathcal{R}}^{n-1} .
\end{aligned}
$$

Proof. Formula (23) is nothing but formula (19). Set $t=0$ in formula (22). If $\mathcal{H}_{H} \in L^{1}\left(S ; \sigma_{\mathcal{R}}^{n-1}\right)$, then we can integrate this formula over $S$. Indeed, under such an assumption, all terms in the formula above turn out to be in $L^{1}\left(S ; \sigma_{\mathcal{R}}^{n-1}\right)$. More precisely, for what concerns the term $\operatorname{div}_{T S}\left(W^{\top}\left|\mathcal{P}_{H} v\right|\right)$, note that $W^{\top} \in \mathfrak{X}^{1}(T S)=\mathbf{C}^{1}(S, T S)$ and that $\left|\mathcal{P}_{H} v\right|$ is Lipschitz continuous. Moreover, if $\mathcal{H}_{H} \in L^{1}\left(S ; \sigma_{\mathcal{R}}^{n-1}\right)$, the second term $\operatorname{div} v_{T S}\left(\langle W, v\rangle v_{H}^{\top}\right)$ belongs to $L^{1}\left(S ; \sigma_{\mathcal{R}}^{n-1}\right)$. In fact, one has

$$
\operatorname{div}_{T S}\left(\langle W, v\rangle v_{H}^{\top}\right)=\operatorname{div}_{T S}\left(\langle W, v\rangle\left(v_{H}-\left|\mathcal{P}_{H} v\right| v\right)\right)
$$

and the claim easily follows by using Lemma 3.1 Hence, we have

$$
I_{S}\left(\sigma_{H}^{n-1}\right)=\int_{S} \dot{\Gamma}(0)=\left.\int_{S} \mathcal{L}_{\widetilde{W}}\left(\sigma_{H}^{n-1}\right)_{t}\right|_{t=0}=\int_{S}\left(-\mathcal{H}_{H}\langle W, v\rangle+\operatorname{div}_{T S}\left(W^{\top}\left|\mathcal{P}_{H} v\right|-\langle W, v\rangle v_{H}^{\top}\right)\right) \sigma_{\mathcal{R}}^{n-1} .
$$


Corollary 4.7. Let the assumptions of Theorem 4.6 hold. Let $\partial S$ be of class $\mathbf{C}^{1}$ and let $\eta$ be the unit outward normal along $\partial S$. Then

$$
I_{S}\left(W, \sigma_{H}^{n-1}\right)=\int_{S}-\mathcal{H}_{H} w \sigma_{H}^{n-1}+\int_{\partial S}\left\langle\left(W^{\top}\left|\mathcal{P}_{H} v\right|-\langle W, v\rangle v_{H}^{\top}\right), \eta\right\rangle \sigma_{\mathcal{R}}^{n-2} .
$$

Furthermore, if $W$ is compactly supported on $S$, then

$$
I_{S}\left(W, \sigma_{H}^{n-1}\right)=\int_{S}-\mathcal{H}_{H} w \sigma_{H}^{n-1} .
$$

Proof. Immediate, since the vector field $Y:=W^{\top}\left|\mathcal{P}_{H} v\right|-\left\langle W^{\perp}, v\right\rangle v_{H}^{\top}$ is admissible (for the Riemannian divergence formula); see condition ( $\bullet$ ) in Definition 2.7. In fact, we see that $Y \in L^{\infty}(S)$ and using the fact that $\mathcal{H}_{H} \in L^{1}\left(S ; \sigma_{\mathcal{R}}^{n-1}\right)$ yields $\operatorname{div}_{T S}(Y) \in L^{1}\left(S ; \sigma_{\mathcal{R}}^{n-1}\right)$.

2nd variation. We regard this proof as a continuation of the proof of the 1st variation formula. From now on, we assume $\mathcal{U}$ and $S$ to be of class $\mathbf{C}^{3}$. Moreover, the boundary $\partial \mathcal{U}$ (or, $\partial S$ when $S$ is compact) is assumed to be of class $\mathbf{C}^{1}$. We also recall that, for the 2 nd variation formula, the variation $\vartheta$ is assumed to be of class $\mathbf{C}^{3}$ on $]-\epsilon, \epsilon[\times \mathcal{U}$.

First, let us compute the second time-derivative of the function $f(t, x)$; see (17). To this end we begin with formula (18). We have

$$
\begin{aligned}
\frac{d^{2} f}{d t^{2}} & =\frac{d}{d t}\left[\frac{d\left|\mathcal{P}_{H_{t}} v^{t}\right|}{d t} \mathcal{J} a c \vartheta_{t}+\left|\mathcal{P}_{H_{t}} v^{t}\right| \frac{d \mathcal{J} a c \vartheta_{t}}{d t}\right] \\
& =\frac{d^{2}\left|\mathcal{P}_{H_{t}} v^{t}\right|}{d t^{2}} \mathcal{J} a c \vartheta_{t}+2 \frac{d\left|\mathcal{P}_{H_{t}} v^{t}\right|}{d t} \frac{d \mathcal{J} a c \vartheta_{t}}{d t}+\left|\mathcal{P}_{H_{t}} v^{t}\right| \frac{d^{2} \mathcal{J} a c \vartheta_{t}}{d t^{2}}
\end{aligned}
$$

At a first glance, it is clear that only the first term is not bounded near the characteristic set $C_{\mathcal{U}}$. More precisely, it is elementary to see that

$$
\frac{d^{2}\left|\mathcal{P}_{H_{t}} v^{t}\right|}{d t^{2}}=\frac{\left|\frac{d \mathcal{P}_{H_{t}} v^{t}}{d t}\right|^{2}-\left\langle\frac{d \mathcal{P}_{H_{t}} v^{t}}{d t}, v_{H}^{t}\right\rangle^{2}}{\left|\mathcal{P}_{H_{t}} v^{t}\right|}+\left\langle\frac{d^{2} \mathcal{P}_{H_{t}} v^{t}}{d t^{2}}, v_{H}^{t}\right\rangle .
$$

This shows that, in order to differentiate under the integral sign, we need the following further hypothesis:

$\left(A_{3}\right)$ there exists $h \in L^{1}\left(\mathcal{U} ; \sigma_{\mathcal{R}}^{n-1}\right)$ such that $\frac{1}{\left|\mathcal{P}_{H_{t}} \nu^{t}\right|} \leq h$ for every $\left.t \in\right]-\epsilon, \epsilon[$.

Remark 4.8. Using $\left(A_{3}\right)$ it is not difficult to show the validity of $\left(A_{2}\right)$ in Warning 4.4

We continue our proof of the 2nd variation of $\sigma_{H}^{n-1}$ with the calculation of $\ddot{\Gamma}(t)$ at a fixed noncharacteristic point $p_{0} \in \mathcal{U} \backslash C_{\mathcal{U}}$. To this end, we start from the following formula:

$$
\left.\left.\ddot{\Gamma}(t)=\vartheta_{t}^{*}\left(\mathcal{L}_{\widetilde{W}}(\widetilde{W}\lrcorner d\left(\sigma_{H}^{n-1}\right)_{t}\right)+\mathcal{L}_{\widetilde{W}} d(\widetilde{W}\lrcorner\left(\sigma_{H}^{n-1}\right)_{t}\right)\right) .
$$

As already said, the 2nd time-derivative of $\Gamma(t)$ can still be computed as a Lie derivative. Moreover, since $d \circ \mathcal{L}=\mathcal{L} \circ d$, we have

$$
\ddot{\Gamma}(t)=\vartheta_{t}^{*}(\underbrace{\left.\mathcal{L}_{\widetilde{W}}(\widetilde{W}\lrcorner d\left(\sigma_{H}^{n-1}\right)_{t}\right)}_{=: A}+d \underbrace{\left.\mathcal{L}_{\widetilde{W}}(\widetilde{W}\lrcorner\left(\sigma_{H}^{n-1}\right)_{t}\right)}_{=: B}) .
$$

The calculation of $\left.A=\mathcal{L}_{\widetilde{W}}(\widetilde{W}\lrcorner d\left(\sigma_{H}^{n-1}\right)_{t}\right)$ is the "hard" part of the 2 nd variation formula and will be done in the sequel. So let us preliminarily consider the quantity $\left.B=\mathcal{L}_{\widetilde{W}}(\widetilde{W}\lrcorner\left(\sigma_{H}^{n-1}\right)_{t}\right)$. (In the next calculations we will use the following general identity for Lie derivatives of a differential form $\omega$ :

$$
\left.\left.\left.\mathcal{L}_{Z}(Y\lrcorner \omega\right)=[Z, Y]\right\lrcorner \omega+Y\right\lrcorner \mathcal{L}_{Z} \omega ;
$$

see [72], Ch. 9, p. 515). We have 


$$
\begin{aligned}
B & \left.=\mathcal{L}_{\widetilde{W}}(\widetilde{W}\lrcorner\left(\sigma_{H}^{n-1}\right)_{t}\right) \\
& \left.=\mathcal{L}_{\widetilde{W}}(\underbrace{\left(\widetilde{W}^{\top}\left|\mathcal{P}_{H_{t}} v^{t}\right|-\left\langle\widetilde{W}, v^{t}\right\rangle v_{H}^{t}\right)}_{=: \widetilde{Y}}\lrcorner\left(\sigma_{\mathcal{R}}^{n-1}\right)_{t}\right) \quad \text { (by Lemma 2.13) } \\
& \left.=[\widetilde{W}, \widetilde{Y}]\lrcorner\left(\sigma_{\mathcal{R}}^{n-1}\right)_{t}+\widetilde{Y}\right\lrcorner \mathcal{L}_{\widetilde{W}}\left(\sigma_{\mathcal{R}}^{n-1}\right)_{t} \quad \text { (by 30) } \\
& \left.\left.=[\widetilde{W}, \widetilde{Y}]^{\top}\right\lrcorner\left(\sigma_{\mathcal{R}}^{n-1}\right)_{t}+\widetilde{Y}\right\lrcorner \underbrace{\left(-\left\langle\widetilde{W}, v^{t}\right\rangle\left(\mathcal{H}_{\mathcal{R}}\right)_{t}+\operatorname{div}_{T} \mathcal{U}_{t}\left(\widetilde{W}^{\top}\right)\right)}_{=: g_{t}}\left(\sigma_{\mathcal{R}}^{n-1}\right)_{t} \quad \text { (by the 1st variation of }\left(\sigma_{\mathcal{R}}^{n-1}\right)_{t}) \\
& \left.=\left([\widetilde{W}, \widetilde{Y}]^{\top}+g_{t} \widetilde{Y}\right)\right\lrcorner\left(\sigma_{\mathcal{R}}^{n-1}\right)_{t} .
\end{aligned}
$$

Therefore, the second term in formula (29), i.e. $d B$, is given by

$$
\left.d B=d\left\{\left([\widetilde{W}, \widetilde{Y}]^{\top}+g_{t} \widetilde{Y}\right)\right\lrcorner\left(\sigma_{\mathcal{R}}^{n-1}\right)_{t}\right\}=\operatorname{div}_{T} \mathcal{U}_{t}\left([\widetilde{W}, \widetilde{Y}]^{\top}+g_{t} \widetilde{Y}\right)\left(\sigma_{\mathcal{R}}^{n-1}\right)_{t}
$$

Step 0. [Divergence-type terms]. Set $t=0$. First, note that $\left.[\widetilde{W}, \widetilde{Y}]^{\top}\right|_{t=0}$ is a vector field of class $\mathbf{C}^{1}$ out of $C_{\mathcal{U}}$. We also stress that

$$
\left.[\widetilde{W}, \widetilde{Y}]^{\top}\right|_{t=0}=\left.\left[\widetilde{W}, \widetilde{W}^{\top}\right]^{\top}\right|_{t=0}-W(\langle W, v\rangle) v_{H}^{\top}-\left.\langle W, v\rangle\left[\widetilde{W}, v_{H}^{t \top}\right]^{\top}\right|_{t=0} .
$$

Clearly, the first term is a vector field of class $\mathbf{C}^{1}$. The second term is the product of a $\mathbf{C}^{1}$ function times the vector field $v_{H}^{\top}$. Although not defined at $C_{\mathcal{U}}$, this term belongs to $L^{\infty}$. Furthermore

$$
\left.\left[\widetilde{W}, v_{H}^{t^{\top}}\right]^{\top}\right|_{t=0}=\left.\left[\widetilde{W},\left(v_{H}^{t}-\left|\mathcal{P}_{H_{t}} v^{t}\right| v^{t}\right)\right]^{\top}\right|_{t=0}=\left.\left[\widetilde{W}, v_{H}^{t}\right]^{\top}\right|_{t=0}-\left.\left|\mathcal{P}_{H} v\right|\left[\widetilde{W}, v^{t}\right]\right|_{t=0}
$$

By using the very definition of $v_{H}^{t}=\frac{\mathcal{P}_{H_{t}} v^{t}}{\left|\mathcal{P}_{H_{t}} v^{t}\right|}$, we easily see that $\left.\left[\widetilde{W}, v_{H}^{t}\right]^{\top}\right|_{t=0}$ can be estimated near the characteristic set $C_{\mathcal{U}}$ by (a constant times) the function $\frac{1}{\left|\mathcal{P}_{H} v\right|}$. Continuing this argument, it is not difficult to realize that the tangential divergence of $[\widetilde{W}, \widetilde{Y}]^{\top}$, at $t=0$, i.e. $\left.\operatorname{div}_{T S}[\widetilde{W}, \widetilde{Y}]^{\top}\right|_{t=0}$, can be estimated, locally around $C_{\mathcal{U}}$, by (a constant times) the function $\frac{1}{\left|\mathcal{P}_{H} v\right|^{2}}$. An analogous argument can be repeated for the second divergence-type term in formula (31). In fact, since the function $g_{t}$ is of class $\mathbf{C}^{1}$ on $\mathcal{U}_{t}$ for all $t \in]-\epsilon, \epsilon$, we see that, near the characteristic set $C_{\mathcal{U}}$, the function $\operatorname{div}_{T} \mathcal{U}\left(g_{0} Y\right)$ can be estimated by (a constant times) the function $\frac{1}{\left|\mathcal{P}_{H} v\right|}$.

Remark 4.9. The previous estimates show that, in order to integrate the divergence-type term $d B$ on $\mathcal{U}$, we need a further condition. More precisely, we have (at least) to require that $\frac{1}{\left|\mathcal{P}_{H} \nu\right|^{2}} \in L^{1}\left(\mathcal{U}, \sigma_{\mathcal{R}}^{n-1}\right)$.

Step 1. We start with the calculation of the term $A$ in formula (29).

Warning 4.10. In order to simplify our calculations, hereafter we shall assume $\mathcal{H}_{H}$ to be constant.

Remark 4.11. We stress that if $\mathcal{H}_{H}=$ const., then $\mathcal{L}_{X} \mathcal{H}_{H}=0$ for all $X \in \mathfrak{X}^{1}(T S)$. In particular, if $W$ denotes the variation vector of $\vartheta_{t}$, we have $\imath^{*}\left(\mathcal{L}_{\widetilde{W}_{H S}}\left(\mathcal{H}_{H}\right)_{t}\right)=\mathcal{L}_{W_{H S}} \mathcal{H}_{H}=0$. Analogously, we have $\imath^{*}\left(\mathcal{L}_{\tau_{\alpha}^{T S}}\left(\mathcal{H}_{H}\right)_{t}\right)=\mathcal{L}_{\tau_{\alpha}^{T S}} \mathcal{H}_{H}=0$ for all $\alpha \in I_{V}$. Hence

$$
\imath^{*}\left(\mathcal{L}_{\tau_{\alpha}}\left(\mathcal{H}_{H}\right)_{t}\right)=\imath^{*}\left(\mathcal{L}_{\varpi_{\alpha}^{t} v_{H}^{t}}\left(\mathcal{H}_{H}\right)_{t}\right) \quad \forall \alpha \in I_{V}
$$


If $t=0$, we have

$$
\begin{aligned}
\left.A\right|_{t=0} & =t^{*}\left(\mathcal{L}_{\widetilde{W}}\left(-w_{t}\left(\mathcal{H}_{H}\right)_{t}\left(\sigma_{H}^{n-1}\right)_{t}\right)\right) \\
& =\left(-w \mathcal{H}_{H} \mathcal{L}_{\widetilde{W}}\left(\sigma_{H}^{n-1}\right)_{t}-W(w) \mathcal{H}_{H}-w \imath^{*}\left(\mathcal{L}_{\widetilde{W}}\left(\mathcal{H}_{H}\right)_{t}\right)\right) \sigma_{H}^{n-1} \\
& =-w \mathcal{H}_{H}\left(-\mathcal{H}_{H}\langle W, v\rangle+\operatorname{div}_{T} \mathcal{U}\left(W^{\top}\left|\mathcal{P}_{H} v\right|-\langle W, v\rangle v_{H}^{\top}\right)\right) \sigma_{\mathcal{R}}^{n-1}-\left(W(w) \mathcal{H}_{H}+w \imath^{*}\left(\mathcal{L}_{\widetilde{W}}\left(\mathcal{H}_{H}\right)_{t}\right)\right) \sigma_{H}^{n-1} \\
& =\left(\mathcal{H}_{H}^{2} w^{2}-W(w) \mathcal{H}_{H}-\imath^{*}\left(\mathcal{L}_{\widetilde{W}}\left(\mathcal{H}_{H}\right)_{t}\right)\right) \sigma_{H}^{n-1}-w \mathcal{H}_{H} \operatorname{div}_{T}\left(W^{\top}\left|\mathcal{P}_{H} v\right|-\langle W, v\rangle v_{H}^{\top}\right) \sigma_{\mathcal{R}}^{n-1},
\end{aligned}
$$

where we have used the 1 st variation of $\sigma_{H}^{n-1}$.

Step 2. Setting $W_{\pitchfork}:=w_{1} v_{H}+W_{V}$, where $W_{V}=\sum_{\alpha \in I_{V}} w_{\alpha} \tau_{\alpha}$, we get that

$$
\begin{aligned}
l^{*}\left(\mathcal{L}_{\widetilde{W}}\left(\mathcal{H}_{H}\right)_{t}\right) & =l^{*}\left(\mathcal{L}_{\widetilde{W}_{H S}}\left(\mathcal{H}_{H}\right)_{t}\right)+i^{*}\left(\mathcal{L}_{\widetilde{W}_{\pitchfork}}\left(\mathcal{H}_{H}\right)_{t}\right) \\
& =l^{*}\left(\mathcal{L}_{\widetilde{W}_{\pitchfork}}\left(\mathcal{H}_{H}\right)_{t}\right) \quad(\text { by Remark 4.11) } \\
& =l^{*}\left(\mathcal{L}_{\widetilde{w}_{1} v_{H}^{t}}\left(\mathcal{H}_{H}\right)_{t}\right)+\sum_{\alpha \in I_{V}} \imath^{*}\left(\mathcal{L}_{\widetilde{w}_{\alpha} \tau_{\alpha}}\left(\mathcal{H}_{H}\right)_{t}\right) \\
& \left.=l^{*}\left(\mathcal{L}_{\widetilde{w}_{1} v_{H}^{t}}\left(\mathcal{H}_{H}\right)_{t}\right)+\sum_{\alpha \in I_{V}} \imath^{*}\left(\mathcal{L}_{\widetilde{w}_{\alpha} \varpi_{\alpha}^{t} v_{H}^{t}}\left(\mathcal{H}_{H}\right)_{t}\right) \quad \text { (by (32) }\right) \\
& =l^{*}\left(\mathcal{L}_{w_{t} v_{H}^{t}}\left(\mathcal{H}_{H}\right)_{t}\right) .
\end{aligned}
$$

Step 3. From Step 2, we see that it remains to calculate $\mathcal{L}_{w_{t}} v_{H}^{t}\left(\mathcal{H}_{H}\right)_{t}=w_{t} \frac{\partial\left(\mathcal{H}_{H}\right)_{t}}{\partial v_{H}^{t}}$. This will be done by using an adapted frame $\underline{\tau}=\left\{\tau_{1}, \ldots, \tau_{n}\right\}$ to $\mathcal{U}$ which satisfies Lemma 3.9 at $p_{0} \in \mathcal{U}$. We also recall that $\tau_{1}(x)=v_{H}(x)$ for every $x \in \mathcal{U}$. We compute

$$
\begin{aligned}
-\frac{\partial\left(\mathcal{H}_{H}\right)_{t}}{\partial v_{H}^{t}} & =\sum_{j \in I_{H S}} \frac{\partial}{\partial \tau_{1}}\left\langle\nabla_{\tau_{j}} \tau_{1}, \tau_{j}\right\rangle \\
& =\sum_{j \in I_{H S}}\left(\left\langle\nabla_{\tau_{1}} \nabla_{\tau_{j}} \tau_{1}, \tau_{j}\right\rangle+\left\langle\nabla_{\tau_{j}} \tau_{1}, \nabla_{\tau_{1}} \tau_{j}\right\rangle\right) \\
& =\sum_{j \in I_{H S}}\left(\left\langle\left(\nabla_{\tau_{1}} \nabla_{\tau_{j}} \tau_{1} \mp \nabla_{\tau_{j}} \nabla_{\tau_{1}} \tau_{1} \mp \nabla_{\left[\tau_{1}, \tau_{j}\right]} \tau_{1}\right), \tau_{j}\right\rangle+\sum_{k=2}^{n}\left\langle\nabla_{\tau_{j}} \tau_{1}, \tau_{k}\right\rangle\left\langle\nabla_{\tau_{1}} \tau_{j}, \tau_{k}\right\rangle\right) \\
& =\sum_{j \in I_{H S}}\left(-\Phi_{1 j}\left(\tau_{1}, \tau_{j}\right)+\left\langle\nabla_{\tau_{j}} \nabla_{\tau_{1}} \tau_{1}, \tau_{j}\right\rangle+\left\langle\nabla_{\left[\tau_{1}, \tau_{j}\right]} \tau_{1}, \tau_{j}\right\rangle+\sum_{\alpha \in I_{V}}^{n}\left\langle\nabla_{\tau_{j}} \tau_{1}, \tau_{\alpha}\right\rangle\left\langle\nabla_{\tau_{1}} \tau_{j}, \tau_{\alpha}\right\rangle\right)
\end{aligned}
$$

where we have used the definition of $\Phi_{1 j}\left(\tau_{1}, \tau_{j}\right)$ and the fact (Lemma 3.9) that $\phi_{j k}=0$ at $p_{0} \in \mathcal{U}$ for every $j, k \in I_{H S}$. We have

$$
\begin{aligned}
\left\langle\nabla_{\left[\tau_{1}, \tau_{j}\right]} \tau_{1}, \tau_{j}\right\rangle & =C_{1 j}^{1} \phi_{1 j}\left(\tau_{1}\right)+\sum_{k \in I_{H S}} C_{1 j}^{k} \phi_{1 j}\left(\tau_{k}\right)+\sum_{\alpha \in I_{V}} C_{1 j}^{\alpha} \phi_{1 j}\left(\tau_{\alpha}\right) \\
& =-\left(\phi_{1 j}\left(\tau_{1}\right)\right)^{2}-\sum_{k \in I_{H S}} \phi_{1 k}\left(\tau_{j}\right) \phi_{1 j}\left(\tau_{k}\right)-\sum_{\alpha \in I_{V}}\left\langle C_{H}^{\alpha} \tau_{1}, \tau_{j}\right\rangle \phi_{1 j}\left(\tau_{\alpha}\right) .
\end{aligned}
$$

Moreover, using (vii) of Lemma 3.9yields

$$
\left\langle\nabla_{\tau_{j}} \tau_{1}, \tau_{\alpha}\right\rangle\left\langle\nabla_{\tau_{1}} \tau_{j}, \tau_{\alpha}\right\rangle=\phi_{1 \alpha}\left(\tau_{j}\right) \phi_{j \alpha}\left(\tau_{1}\right)=-\frac{1}{4}\left\langle C_{H}^{\alpha} \tau_{1}, \tau_{j}\right\rangle^{2}
$$

Therefore, Lemma 3.8 implies that

$$
-\frac{\partial\left(\mathcal{H}_{H}\right)_{t}}{\partial v_{H}^{t}}=\frac{1}{2} \sum_{\alpha \in I_{H_{2}}}\left|C_{H}^{\alpha} \tau_{1}\right|^{2}+\operatorname{div}_{H S_{t}}\left(\nabla_{\tau_{1}} \tau_{1}\right)-\sum_{j, k \in I_{H S}}\left(\left(\phi_{1 j}\left(\tau_{1}\right)\right)^{2}+\phi_{1 k}\left(\tau_{j}\right) \phi_{1 j}\left(\tau_{k}\right)+\left\langle C_{H}^{\alpha} \tau_{1}, \tau_{j}\right\rangle \phi_{1 j}\left(\tau_{\alpha}\right)\right) .
$$


Hence, from Lemma 3.5, Lemma 4.2 and formula (15) we get that

$$
\begin{aligned}
& -\frac{\partial\left(\mathcal{H}_{H}\right)_{t}}{\partial v_{H}^{t}}=\frac{1}{2} \sum_{\alpha \in I_{H_{2}}}\left|C_{H}^{\alpha} \tau_{1}\right|^{2}-\operatorname{div}_{H S_{t}}\left(\frac{\operatorname{grad}_{H S_{t}} w_{t}}{w_{t}}+C_{H}\left(\varpi_{H_{2}}^{t}\right) \tau_{1}\right) \\
& -\left|\frac{\operatorname{grad}_{H S_{t}} w_{t}}{w_{t}}+C_{H}\left(\varpi_{H_{2}}^{t}\right) \tau_{1}\right|^{2}+\left\|A_{H}^{t}\right\|_{\mathrm{Gr}}^{2}-\left\|S_{H}^{t}\right\|_{\mathrm{G} r}^{2} \\
& -\sum_{j \in I_{H S}}\left\langle C_{H}^{\alpha} \tau_{1}, \tau_{j}\right\rangle\left(\frac{1}{2}\left\langle C_{H}^{\alpha} \tau_{1}, \tau_{j}\right\rangle-\left\langle C\left(\varpi^{t}\right) \tau_{\alpha}, \tau_{j}\right\rangle+\tau_{j}\left(\varpi_{\alpha}^{t}\right)-\varpi_{\alpha}^{t} \frac{\tau_{j}\left(w_{t}\right)}{w_{t}}\right) . \\
& =-\operatorname{div}_{H S_{t}}\left(\frac{\operatorname{grad}_{H S_{t}} w_{t}}{w_{t}}+C_{H}\left(\varpi_{H_{2}}^{t}\right) \tau_{1}\right)-\left|\frac{\operatorname{grad}_{H S_{t}} w_{t}}{w_{t}}+C_{H}\left(\varpi_{H_{2}}^{t}\right) \tau_{1}\right|^{2}+\left\|A_{H}^{t}\right\|_{\mathrm{G} r}^{2}-\left\|S_{H}^{t}\right\|_{\mathrm{G} r}^{2} \\
& +\sum_{j \in I_{H S} \alpha \in I_{V}}\left\langle C_{H}^{\alpha} \tau_{1}, \tau_{j}\right\rangle\left(\left\langle C\left(\varpi^{t}\right) \tau_{\alpha}, \tau_{j}\right\rangle-\tau_{j}\left(\varpi_{\alpha}^{t}\right)+\varpi_{\alpha}^{t} \frac{\tau_{j}\left(w_{t}\right)}{w_{t}}\right) \\
& =-\operatorname{div}_{H S_{t}}\left(\frac{\operatorname{grad}_{H S_{t}} w_{t}}{w_{t}}+C_{H}\left(\varpi_{H_{2}}^{t}\right) \tau_{1}\right)-\left|\frac{\operatorname{grad}_{H S_{t}} w_{t}}{w_{t}}+C_{H}\left(\varpi_{H_{2}}^{t}\right) \tau_{1}\right|^{2}+\left\|A_{H}^{t}\right\|_{\mathrm{G} r}^{2}-\left\|S_{H}^{t}\right\|_{\mathrm{G} r}^{2} \\
& +\sum_{\alpha \in I_{V}}\left(\left\langle C_{H}^{\alpha} \tau_{1}, C\left(\varpi^{t}\right) \tau_{\alpha}\right\rangle-\left\langle C_{H}^{\alpha} \tau_{1}, \operatorname{grad}_{H S_{t}} \varpi_{\alpha}^{t}\right\rangle\right)+\left\langle C_{H}\left(\varpi_{H_{2}}^{t}\right) \tau_{1}, \frac{\operatorname{grad}_{H S_{t}} w_{t}}{w_{t}}\right\rangle \\
& =-\frac{\Delta H S_{t} w_{t}}{w_{t}}-\operatorname{div}_{H S_{t}}\left(C_{H}\left(\varpi_{H_{2}}^{t}\right) \tau_{1}\right)-\left|C_{H}\left(\varpi_{H_{2}}^{t}\right) \tau_{1}\right|^{2}-2\left\langle\frac{\operatorname{grad}_{H S_{t}} w_{t}}{w_{t}}, C_{H}\left(\varpi_{H_{2}}^{t}\right) \tau_{1}\right\rangle+\left\|A_{H}^{t}\right\|_{\mathrm{G} r}^{2}-\left\|S_{H}^{t}\right\|_{\mathrm{G} r}^{2} \\
& +\sum_{\alpha \in I_{V}}\left(\left\langle C_{H}^{\alpha} \tau_{1}, C\left(\varpi^{t}\right) \tau_{\alpha}\right\rangle-\left\langle C_{H}^{\alpha} \tau_{1}, \operatorname{grad}_{H S_{t}} \varpi_{\alpha}^{t}\right\rangle\right)+\left\langle C_{H}\left(\varpi_{H_{2}}^{t}\right) \tau_{1}, \frac{\operatorname{grad}_{H S_{t}} w_{t}}{w_{t}}\right\rangle \\
& =-\frac{\Delta H S_{t} w_{t}}{w_{t}}-\operatorname{div}_{H S_{t}}\left(C_{H}\left(\varpi_{H_{2}}^{t}\right) \tau_{1}\right)-\left|C_{H}\left(\varpi_{H_{2}}^{t}\right) \tau_{1}\right|^{2}-\left\langle\frac{\operatorname{grad}_{H S_{t}} w_{t}}{w_{t}}, C_{H}\left(\varpi_{H_{2}}^{t}\right) \tau_{1}\right\rangle+\left\|A_{H}^{t}\right\|_{\mathrm{G} r}^{2}-\left\|S_{H}^{t}\right\|_{\mathrm{G} r}^{2} \\
& +\sum_{\alpha \in I_{V}}\left(\left\langle C_{H}^{\alpha} \tau_{1}, C\left(\varpi^{t}\right) \tau_{\alpha}\right\rangle-\left\langle C_{H}^{\alpha} \tau_{1}, \operatorname{grad}_{H S_{t}} \varpi_{\alpha}^{t}\right\rangle\right) \\
& =-\frac{\mathcal{L}_{H S_{t}} w_{t}}{w_{t}}-\mathcal{D}_{H S_{t}}\left(C_{H}\left(\varpi_{H_{2}}^{t}\right) \tau_{1}\right)+\left\|A_{H}^{t}\right\|_{\mathrm{G} r}^{2}-\left\|S_{H}^{t}\right\|_{\mathrm{G} r}^{2}+\sum_{\alpha \in I_{V}}\left(\left\langle C_{H}^{\alpha} \tau_{1}, C\left(\varpi^{t}\right) \tau_{\alpha}\right\rangle-\left\langle C_{H}^{\alpha} \tau_{1}, \operatorname{grad}_{H S_{t}} \varpi_{\alpha}^{t}\right\rangle\right) .
\end{aligned}
$$

Under the assumptions made in Warning 4.4, Remark 4.8 and Warning 4.10, we can now achieve the proof of the 2nd variation of $\sigma_{H}^{n-1}$.

Step 4. We know by Remark 2.9 that if $\frac{1}{\left|\mathcal{P}_{H} \nu\right|^{2}} \in L^{2}\left(\mathcal{U}, \sigma_{H}^{n-1}\right)$, then the function $w^{2}$ is admissible; see Definition 2.7. Hence, if $\frac{1}{\left|\mathcal{P}_{H} v\right|^{2}} \in L^{2}\left(S ; \sigma_{H}^{n-1}\right)$, then

$$
\begin{aligned}
I I_{\mathcal{U}}\left(W, \sigma_{H}^{n-1}\right)= & \int_{\mathcal{U}}\left(\left(w \mathcal{H}_{H}\right)^{2}-W(w) \mathcal{H}_{H}-w \iota^{*}\left(\mathcal{L}_{w_{t}} v_{H}^{t}\left(\mathcal{H}_{H}\right)_{t}\right)\right) \sigma_{H}^{n-1} \\
& +\int_{\mathcal{U}}\left(\operatorname{div}_{T \mathcal{U}}\left(\left.[\widetilde{W}, \widetilde{Y}]^{\top}\right|_{t=0}+g_{0} Y\right)-w \mathcal{H}_{H} \operatorname{div}_{T \mathcal{U}}\left(W^{\top}\left|\mathcal{P}_{H} v\right|-\langle W, v\rangle v_{H}^{\top}\right)\right) \sigma_{\mathcal{R}}^{n-1} \\
= & \int_{\mathcal{U}}\left\{-W(w) \mathcal{H}_{H}+w^{2}\left(\left(\mathcal{H}_{H}\right)^{2}+\left\|A_{H}\right\|_{\mathrm{G} r}^{2}-\left\|S_{H}\right\|_{\mathrm{G} r}^{2}\right)-w \mathcal{L}_{H S} w\right. \\
& \left.+w^{2}\left[-\mathcal{D}_{H S}\left(C_{H}\left(\varpi_{H_{2}}\right) \tau_{1}\right)+\sum_{\alpha \in I_{V}}\left(\left\langle C_{H}^{\alpha} \tau_{1}, C(\varpi) \tau_{\alpha}\right\rangle-\left\langle C_{H}^{\alpha} \tau_{1}, \operatorname{grad}_{H S} \varpi_{\alpha}\right\rangle\right)\right]\right\} \sigma_{H}^{n-1} \\
& +\int_{\mathcal{U}}\left(\operatorname{div}_{T \mathcal{U}}\left(\left.[\widetilde{W}, \widetilde{Y}]^{\top}\right|_{t=0}+g_{0} Y\right)-w \mathcal{H}_{H} \operatorname{div}_{T \mathcal{U}}\left(W^{\top}\left|\mathcal{P}_{H} v\right|-\langle W, v\rangle v_{H}^{\top}\right)\right) \sigma_{\mathcal{R}}^{n-1}
\end{aligned}
$$




$$
\begin{aligned}
= & \int_{\mathcal{U}}\left\{-W(w) \mathcal{H}_{H}+w^{2}\left(\left(\mathcal{H}_{H}\right)^{2}+\left\|A_{H}\right\|_{\mathrm{G} r}^{2}-\left\|S_{H}\right\|_{\mathrm{G} r}^{2}\right)+\left|\operatorname{grad}_{H S} w\right|^{2} \quad \text { (by formula (6) }\right) \\
& \left.+w^{2} \sum_{\alpha \in I_{V}}\left(-\varpi_{\alpha} \mathcal{D}_{H S}\left(C_{H}^{\alpha} \tau_{1}\right)+\left\langle C_{H}^{\alpha} \tau_{1}, C(\varpi) \tau_{\alpha}\right\rangle-2\left\langle C_{H}^{\alpha} \tau_{1}, \operatorname{grad}_{H S} \varpi_{\alpha}\right\rangle\right)\right\} \sigma_{H}^{n-1} \\
& +\int_{\mathcal{U}}\left(\operatorname{div}_{T \mathcal{U}}\left(\left.[\widetilde{W}, \widetilde{Y}]^{\top}\right|_{t=0}+g_{0} Y\right)-w \mathcal{H}_{H} \operatorname{div}_{T \mathcal{U}}\left(W^{\top}\left|\mathcal{P}_{H} v\right|-\langle W, v\rangle v_{H}^{\top}\right)\right) \sigma_{\mathcal{R}}^{n-1} .
\end{aligned}
$$

Using Lemma 3.6 yields

$$
\begin{aligned}
& \int_{\mathcal{U}}\left\{-W(w) \mathcal{H}_{H}+w^{2}\left(\left(\mathcal{H}_{H}\right)^{2}-\left\|A_{H}\right\|_{\mathrm{G} r}^{2}-\left\|S_{H}\right\|_{\mathrm{Gr}}^{2}\right)+\left|\operatorname{grad}_{H S} w\right|^{2}\right. \\
& \left.+w^{2} \sum_{\alpha \in I_{V}}\left[-\left|C_{H}\left(\varpi_{H_{2}}\right) \tau_{1}\right|^{2}+\left\langle C_{H}^{\alpha} \tau_{1}, C(\varpi) \tau_{\alpha}\right\rangle-2\left\langle C_{H}^{\alpha} \tau_{1}, \operatorname{grad}_{H S} \varpi_{\alpha}\right\rangle\right]\right\} \sigma_{H}^{n-1} \\
= & \int_{\mathcal{U}}\left\{-W(w) \mathcal{H}_{H}+w^{2}\left(\left(\mathcal{H}_{H}\right)^{2}-\left\|A_{H}\right\|_{\mathrm{G} r}^{2}-\left\|S_{H}\right\|_{\mathrm{G} r}^{2}\right)+\left|\operatorname{grad}_{H S} w\right|^{2}\right. \\
& \left.-w^{2} \sum_{\alpha \in I_{V}}\left\langle\left(2 \operatorname{grad}_{H S}\left(\varpi_{\alpha}\right)-C(\varpi) \tau_{\alpha}^{T S}\right), C^{\alpha} \tau_{1}\right\rangle\right\} \sigma_{H}^{n-1},
\end{aligned}
$$

where we recall that $\tau_{\alpha}^{T S}=\tau_{\alpha}-\varpi_{\alpha} \tau_{1}$ and that $\tau_{1}=v_{H}, \tau_{\alpha}=X_{\alpha}$ for any $\alpha \in I_{V}$; see Definition 1.9 . Finally, using the last identity in (34) yields the following:

Theorem 4.12 (2nd variation of $\sigma_{H}^{n-1}$ ). Let $S \subset \mathbb{G}$ be a compact hypersurface of class $\mathbf{C}^{3}$ with, or without, boundary and let $\vartheta:]-\epsilon, \epsilon\left[\times S \rightarrow \mathbb{G}\right.$ be a $\mathbf{C}^{3}$-smooth variation of $S$. Let $W=\left.\frac{d \vartheta_{t}}{d t}\right|_{t=0}$ be the variation vector field, let $w:=\frac{\left\langle W^{\perp}, v\right\rangle}{\left|\mathcal{P}_{H} v\right|}$ and let $W^{\perp}, W^{\top}$ be the normal and tangential components of $W$ along $S$, respectively. We further assume that:

(i) there exist $\mathrm{8}^{8} h \in L^{1}\left(\mathcal{U} ; \sigma_{\mathcal{R}}^{n-1}\right)$ such that $\frac{1}{\left|\mathcal{P}_{H_{t}} \nu^{t}\right|} \leq h$ for every $\left.t \in\right]-\epsilon, \epsilon[$

(ii) the horizontal mean curvature $\mathcal{H}_{H}$ of $S$ is constant;

(iii) the function $\frac{1}{\left|\mathcal{P}_{H} v\right|^{2}} \in L^{2}\left(S ; \sigma_{H}^{n-1}\right)$.

Then

$$
\begin{aligned}
I I_{S}\left(W, \sigma_{H}^{n-1}\right)= & \int_{S}\left\{-W(w) \mathcal{H}_{H}+w^{2}\left(\left(\mathcal{H}_{H}\right)^{2}-\left\|A_{H}\right\|_{\mathrm{G} r}^{2}-\left\|S_{H}\right\|_{\mathrm{Gr}}^{2}\right)+\left|\operatorname{grad}_{H S} w\right|^{2}\right. \\
& \left.-w^{2} \sum_{\alpha \in I_{V}}\left\langle\left(2 \operatorname{grad}_{H S}\left(\varpi_{\alpha}\right)-C(\varpi) \tau_{\alpha}^{T S}\right), C^{\alpha} v_{H}\right\rangle\right\} \sigma_{H}^{n-1} \\
& +\int_{S}\left\{\operatorname{div}_{T S}\left(\left.[\widetilde{W}, \widetilde{Y}]^{\top}\right|_{t=0}+g_{0} Y\right)-w \mathcal{H}_{H} \operatorname{div}_{T S}\left(W^{\top}\left|\mathcal{P}_{H} v\right|-\langle W, v\rangle v_{H}^{\top}\right)\right\} \sigma_{\mathcal{R}}^{n-1}
\end{aligned}
$$

where $\widetilde{Y}:=\widetilde{W}^{\top}\left|\mathcal{P}_{H_{t}} v^{t}\right|-\left\langle\widetilde{W}, v^{t}\right\rangle v_{H}^{t^{\top}}, Y=\left.\widetilde{Y}\right|_{t=0}$ and $g_{0}=\left(-\left\langle W^{\perp}, v\right\rangle \mathcal{H}_{\mathcal{R}}+\operatorname{div}_{T S} W^{\top}\right)$.

Proof. If $C_{S} \neq \emptyset$, then (i) implies the possibility to differentiate under the integral sign the function $f(t, x)$ defined by formula (17). This has been done by using the machinery of differential forms. This way we have obtained (35) by further assuming that $\mathcal{H}_{H}$ is constant. Nevertheless, we have to take care of the existence of the involved integrals. The integrability of the divergence-type terms has been already discussed at Step 0. We recall that if $\frac{1}{\left|\mathcal{P}_{H} \nu\right|^{2}} \in L^{1}\left(S ; \sigma_{\mathcal{R}}^{n-1}\right)$ it follows that all these terms are integrable. Clearly, the latter condition is automatically implied by (iii). Moreover, the condition $\frac{1}{\left|\mathcal{P}_{H} \nu\right|^{2}} \in L^{2}\left(S, \sigma_{H}^{n-1}\right)$ implies that function $w^{2}$ is admissible; see Definition 2.7 Hence, using formula (6), we see that the function $-w \mathcal{L}_{H S} w$ can be integrated by parts, as previously done. Furthermore, a rather tedious (but completely elementary) analysis shows that the same condition implies that each term in (35) is integrable

\footnotetext{
${ }^{8}$ Alternatively, we can assume the validity of $\left(A_{2}\right)$ in Warning 4.4
} 
over $S$. More precisely, the integral of each of these terms can be estimated, near the characteristic set $C_{S}$, by (a constant times) $\int_{S} \frac{1}{\left|\mathcal{P}_{H} v\right|^{4}} \sigma_{H}^{n-1}$. (Note that these estimates follow basically from the same calculation performed in formula (11) of Remark 3.2 In particular, one uses the following

$$
X\left|\mathcal{P}_{H} v\right|=\frac{\left\langle\left[\mathcal{J}_{\mathcal{R}}\left(\mathcal{P}_{H} v\right)\right]^{T r} \mathcal{P}_{H} v, X\right\rangle}{\left|\mathcal{P}_{H} v\right|}
$$

for every $X \in \mathfrak{X}(\mathbb{G})$. An analogous argument was made at Step 0). This achieves the proof.

Corollary 4.13. Let the assumptions of Theorem 4.12 hold and let $\vartheta$ be compactly supported on $S$. If $S$ is $H$-minimal, i.e. $\mathcal{H}_{H}=0$, then

$I_{S}\left(W, \sigma_{H}^{n-1}\right)=\int_{S}\left\{\left|\operatorname{grad}_{H S} w\right|^{2}-w^{2}\left(\left\|A_{H}\right\|_{\mathrm{G} r}^{2}+\left\|S_{H}\right\|_{\mathrm{G} r}^{2}+\sum_{\alpha \in I_{V}}\left\langle\left(2 \operatorname{grad}_{H S}\left(\varpi_{\alpha}\right)-C(\varpi) \tau_{\alpha}^{T S}\right), C^{\alpha} v_{H}\right\rangle\right)\right\} \sigma_{H}^{n-1}$.

Proof. We just have to analyze the 2nd integral in formula (35). We already know that $Y$ is admissible; see Corollary 4.7. Since $g_{0}$ is $\mathbf{C}^{1}$-smooth on $S$ and $g_{0}=0$ on $\partial S$, we can conclude that $g_{0} Y$ is admissible. Hence $\int_{S} \operatorname{div}_{T S}\left(g_{0} Y\right) \sigma_{\mathcal{R}}^{n-1}=\int_{\partial S}\left\langle g_{0} Y, \eta\right\rangle \sigma_{\mathcal{R}}^{n-2}=0$. Furthermore, since $\mathcal{H}_{H}=0$ and $\left.[\widetilde{W}, \widetilde{Y}]^{\top}\right|_{t=0}=0$ on $\partial S$, we just have to show that $\left.[\widetilde{W}, \widetilde{Y}]^{\top}\right|_{t=0}$ is admissible. More precisely, below we shall prove that $\left.[\widetilde{W}, \widetilde{Y}]^{\top}\right|_{t=0}$ satisfies the assumptions in Proposition 2.11. Under our assumptions, this can be seen as follows. First, note that $\widetilde{W}$ is of class $\mathbf{C}^{2}$ on $]-\epsilon, \epsilon\left[\times S\right.$ and that $\widetilde{Y}=\widetilde{W}^{\top}\left|\mathcal{P}_{H_{t}} v^{t}\right|-\left\langle\widetilde{W}, v^{t}\right\rangle v_{H}^{t}{ }^{\top}$ is of class $\mathbf{C}^{1}$ on $]-\epsilon, \epsilon\left[\times\left(S \backslash C_{S}\right)\right.$. Moreover, we have

$$
\begin{aligned}
{\left.[\widetilde{W}, \widetilde{Y}]^{\top}\right|_{t=0} } & =\left.\left(\nabla_{\widetilde{W}} \widetilde{Y}-\nabla_{\widetilde{Y}} \widetilde{W}\right)\right|_{t=0} \\
& =\left.\left[\left|\mathcal{P}_{H_{t}} v^{t}\right| \nabla_{\widetilde{W}} \widetilde{W}^{\top}+\widetilde{W}\left(\left|\mathcal{P}_{H_{t}} v^{t}\right|\right) \widetilde{W}^{\top}-\left(\widetilde{W}\left(\left\langle\widetilde{W}, v^{t}\right\rangle\right) v_{H}^{t}+\left\langle\widetilde{W}, v^{t}\right\rangle \nabla_{\widetilde{Y}} v_{H}^{t}\right)\right]\right|_{t=0}
\end{aligned}
$$

We claim that $\left.[\widetilde{W}, \widetilde{Y}]^{\top}\right|_{t=0} \in W_{\text {comp }}^{1,2}(S ; T S)$. In fact, the first addend is Lipschitz, the second and third addends are in $L^{\infty}$, and the fourth addend can be estimated by (a constant times) $\frac{1}{\left|\mathcal{P}_{H}\right|}$. It is worth remarking that the estimate of the fourth addend relies on the fact that

$$
\mathcal{J}_{\mathcal{R}} v_{H}=\mathcal{J}_{\mathcal{R}}\left(\frac{\mathcal{P}_{H} v}{\left|\mathcal{P}_{H} v\right|}\right)=\frac{\mathcal{J}_{\mathcal{R}} \mathcal{P}_{H} v-v_{H} \otimes \operatorname{grad}_{\mathcal{R}}\left|\mathcal{P}_{H} v\right|}{\left|\mathcal{P}_{H} v\right|}
$$

In particular, under our assumptions, we have $\frac{1}{\left|\mathcal{P}_{H} v\right|} \in L^{2}\left(S ; \sigma_{\mathcal{R}}^{n-1}\right)$. Hence, $\left.[\widetilde{W}, \widetilde{Y}]^{\top}\right|_{t=0} \in L^{2}\left(S ; \sigma_{\mathcal{R}}^{n-1}\right)$. Continuing this argument, we easily see that each tangential derivative of $\left.[\widetilde{W}, \widetilde{Y}]^{\top}\right|_{t=0}$ can be estimated by (a constant times) $\frac{1}{\left|\mathcal{P}_{H} v\right|^{2}}$ and the claim follows since $\frac{1}{\left|\mathcal{P}_{H} v\right|^{2}} \in L^{2}\left(S ; \sigma_{\mathcal{R}}^{n-1}\right)$.

Notation 4.14. For the sake of simplicity, we shall set:

$$
\mathcal{B}_{T S}:=\underbrace{\left\|S_{H}\right\|_{\mathrm{G} r}^{2}+\left\|A_{H}\right\|_{\mathrm{G} r}^{2}}_{=\left\|B_{H}\right\|_{\mathrm{G} r}^{2}}+\sum_{\alpha \in I_{V}}\left\langle\left(2 \operatorname{grad}_{H S}\left(\varpi_{\alpha}\right)-C(\varpi) \tau_{\alpha}^{T S}\right), C^{\alpha} \tau_{1}\right\rangle .
$$

We stress that, unlike the Euclidean case where $\mathcal{B}_{T S}:=\left\|B_{\mathcal{R}}\right\|_{\mathrm{Gr}}^{2}$, it is not necessarily true that $\mathcal{B}_{T S} \geq 0$; an example of this fact can be found in Section 6.2, see Remark 6.10

Remark 4.15 (Heisenberg group; see Example 1.8). Let $S \subset \mathbb{H}^{n}$ be $H$-minimal and set $v_{H}^{\circ}:=-C_{H}^{2 n+1} v_{H}$. Then, we have

$$
\mathcal{B}_{T S}=\left\|S_{H}\right\|_{\mathrm{G} r}^{2}-\left(2 \frac{\partial \varpi}{\partial v_{H}^{\circ}}-\frac{n+1}{2} \varpi^{2}\right)
$$




\section{GeOMETRIC IDENTITIES FOR CONSTANT $H$-MEAN CURVATURE HYPERSURFACES}

Lemma 5.1. Let $S \subset \mathbb{G}$ be a hypersurface of class $\mathbf{C}^{2}$ and let $\phi \in \mathbf{C}^{2}(\mathbb{G})$. Then we have

$$
\Delta_{H S} \phi=\Delta_{H} \phi+\mathcal{H}_{H} \frac{\partial \phi}{\partial v_{H}}-\left\langle\operatorname{Hess}_{H} \phi v_{H}, v_{H}\right\rangle
$$

at each non-characteristic point $p \in S \backslash C_{S}$.

Proof. First, note that we can use the invariant definition of the Laplacian on vector bundles; see, for instance, [15]. So we have

$$
\begin{aligned}
\Delta_{H} \phi & =\sum_{i \in I_{H}}\left(\tau_{i}^{(2)}-\nabla_{\tau_{i}}^{H} \tau_{i}\right)(\phi) \\
& =\tau_{1}^{(2)}(\phi)-\left(\nabla_{\tau_{1}}^{H} \tau_{1}\right)(\phi)+\sum_{i \in I_{H S}}\left(\left(\tau_{i}^{(2)}-\nabla_{\tau_{i}}^{H S} \tau_{i}\right)(\phi)-\left\langle\nabla_{\tau_{i}}^{H} \tau_{i}, v_{H}\right\rangle \frac{\partial \phi}{\partial v_{H}}\right) \\
& =\tau_{1}^{(2)}(\phi)-\left(\nabla_{\tau_{1}}^{H} \tau_{1}\right)(\phi)+\Delta_{H S} \phi-\mathcal{H}_{H} \frac{\partial \phi}{\partial v_{H}} .
\end{aligned}
$$

Now we claim that $\tau_{1}^{(2)}(\phi)-\left(\nabla_{\tau_{1}}^{H} \tau_{1}\right)(\phi)=\left\langle\operatorname{Hess}_{H}(\phi) v_{H}, v_{H}\right\rangle$. To prove this claim, set $\tau_{1}=\sum_{i \in I_{H}} A_{i}^{1} X_{i}$ and compute

$$
\tau_{1}^{(2)}(\phi)=\sum_{i \in I_{H}}\left(\tau_{1}\left(A_{i}^{1} X_{i}(\phi)\right)\right)=\sum_{i, j \in I_{H}}\left(\tau_{1}\left(A_{i}^{1}\right) X_{i}(\phi)+A_{i}^{1} A_{j}^{1} X_{j}\left(X_{i}(\phi)\right)\right) .
$$

Since $\nabla_{\tau_{1}}^{H} \tau_{1}=\sum_{i, j \in I_{H}}(\tau_{1}\left(A_{i}^{1}\right) X_{i}+A_{i}^{1} A_{j}^{1} \underbrace{\nabla_{X_{i}}^{H} X_{j}}_{=0})$, we get that

$$
\tau_{1}^{(2)}(\phi)-\left(\nabla_{\tau_{1}}^{H} \tau_{1}\right)(\phi)=\sum_{i, j \in I_{H}} A_{i}^{1} A_{j}^{1} X_{j}\left(X_{i}(\phi)\right)=\left\langle\operatorname{Hess}_{H}(\phi) v_{H}, v_{H}\right\rangle
$$

as wished.

Lemma 5.2. Let $S \subset \mathbb{G}$ be a $\mathbf{C}^{2}$ non-characteristic hypersurface of constant horizontal mean curvature $\mathcal{H}_{H}$. Then, the following identities hold:

(i) $\sum_{i \in I_{H S}}\left\langle\nabla_{\tau_{i}}^{H} \nabla_{\tau_{i}}^{H} v_{H}, v_{H}\right\rangle=-\left\|B_{H}\right\|_{\mathrm{G} r}^{2}$;

(ii) $\sum_{i \in I_{H S}}\left\langle\nabla_{\tau_{i}}^{H} \nabla_{\tau_{i}}^{H} \nu_{H}, \tau_{k}\right\rangle=-\left(\left\langle\nabla_{\nu_{H}}^{H} \nu_{H}, C_{H S}\left(\varpi_{H_{2}}\right) \tau_{k}\right\rangle+\sum_{\alpha \in I_{V}}\left\langle C_{H}^{\alpha} \operatorname{grad}_{H S} \varpi_{\alpha}, \tau_{k}\right\rangle+\mathcal{H}_{H}\left\langle C_{H}\left(\varpi_{H_{2}}\right) v_{H}, \tau_{k}\right\rangle-\right.$ $\left.B_{H}\left(C_{H}\left(\varpi_{H_{2}}\right) v_{H}, \tau_{k}\right)\right) \quad \forall k \in I_{H S}$.

Proof. Throughout this proof, we use an adapted frame as in Lemma3.9. Fix a point $p \in S$.

Proof of $(i)$. Since $\left\langle v_{H}, v_{H}\right\rangle=1$ we get that $\left\langle\nabla_{\tau_{i}}^{H} v_{H}, v_{H}\right\rangle=0 \forall i \in I_{H S}$. So, we have

$$
\begin{aligned}
\sum_{i \in I_{H S}}\left\langle\nabla_{\tau_{i}}^{H} \nabla_{\tau_{i}}^{H} \nu_{H}, v_{H}\right\rangle & =-\sum_{i \in I_{H S}}\left\langle\nabla_{\tau_{i}}^{H} v_{H}, \nabla_{\tau_{i}}^{H} v_{H}\right\rangle \\
=-\sum_{i, j, k \in I_{H S}}\left\langle\nabla_{\tau_{i}}^{H} v_{H}, \tau_{j}\right\rangle\left\langle\nabla_{\tau_{i}}^{H} \nu_{H}, \tau_{k}\right\rangle\left\langle\tau_{j}, \tau_{k}\right\rangle & =-\sum_{i, j \in I_{H S}}\left\langle\nabla_{\tau_{i}}^{H} v_{H}, \tau_{j}\right\rangle^{2}=-\left\|B_{H}\right\|_{\mathrm{G} r}^{2} .
\end{aligned}
$$

Proof of (ii). Since $\left\langle v_{H}, \tau_{k}\right\rangle=0$ for any $k \in I_{H S}$ we get that $\left\langle\nabla_{\tau_{i}}^{H} v_{H}, \tau_{k}\right\rangle=-\left\langle v_{H}, \nabla_{\tau_{i}}^{H} \tau_{k}\right\rangle$ for every $i \in I_{H S}$. Therefore

$$
\left\langle\nabla_{\tau_{i}}^{H} \nabla_{\tau_{i}}^{H} v_{H}, \tau_{k}\right\rangle+\left\langle\nabla_{\tau_{i}}^{H} v_{H}, \nabla_{\tau_{i}}^{H} \tau_{k}\right\rangle=-\left\langle\nabla_{\tau_{i}}^{H} v_{H}, \nabla_{\tau_{i}}^{H} \tau_{k}\right\rangle-\left\langle v_{H}, \nabla_{\tau_{i}}^{H} \nabla_{\tau_{i}}^{H} \tau_{k}\right\rangle
$$


Note that $\nabla_{\tau_{i}}^{H} v_{H} \in H S$ and that, by our choice of the moving frame, we have $\left(\nabla_{\tau_{i}}^{H S} \tau_{k}\right)(p)=0$. Hence

$$
\begin{aligned}
A_{i}:=\left\langle\nabla_{\tau_{i}}^{H} \nabla_{\tau_{i}}^{H} v_{H}, \tau_{k}\right\rangle & =-\left\langle v_{H}, \nabla_{\tau_{i}}^{H} \nabla_{\tau_{i}}^{H} \tau_{k}\right\rangle \\
& =-\left\langle v_{H}, \nabla_{\tau_{i}}^{H}\left(\nabla_{\tau_{k}}^{H} \tau_{i}+\left[\tau_{i}, \tau_{k}\right]_{H}\right)\right\rangle \\
& =-\left\langle v_{H}, \nabla_{\tau_{i}}^{H} \nabla_{\tau_{k}}^{H} \tau_{i}\right\rangle-\left\langle v_{H}, \nabla_{\tau_{i}}^{H}\left(\left\langle\left[\tau_{i}, \tau_{k}\right]_{H}, v_{H}\right\rangle v_{H}\right)\right\rangle \quad \text { (by Lemma3.9] } \\
& =-\left\langle v_{H}, \nabla_{\tau_{i}}^{H} \nabla_{\tau_{k}}^{H} \tau_{i}\right\rangle-\tau_{i}\left(\left\langle\left[\tau_{i}, \tau_{k}\right]_{H}, v_{H}\right\rangle\right) \quad \forall i, k \in I_{H S} .
\end{aligned}
$$

Now since $\left\langle\left[\tau_{i}, \tau_{k}\right]_{H}, v_{\mathcal{R}}\right\rangle=\left\langle\left[\tau_{i}, \tau_{k}\right], v_{\mathcal{R}}\right\rangle=0$, we get that

$$
\left\langle\left[\tau_{i}, \tau_{k}\right], v_{H}\right\rangle=-\sum_{\alpha \in I_{V}} \varpi_{\alpha}\left\langle\left[\tau_{i}, \tau_{k}\right], \tau_{\alpha}\right\rangle=-\sum_{\alpha \in I_{V}} \varpi_{\alpha} C_{i k}^{\alpha}=\sum_{\alpha \in I_{V}} \varpi_{\alpha}\left\langle C_{H}^{\alpha} \tau_{i}, \tau_{k}\right\rangle=\left\langle C_{H S}\left(\varpi_{H_{2}}\right) \tau_{i}, \tau_{k}\right\rangle \quad \forall i, k \in I_{H S} .
$$

Hence $A_{i}=-\left\langle v_{H}, \nabla_{\tau_{i}}^{H} \nabla_{\tau_{k}}^{H} \tau_{i}\right\rangle-\tau_{i}\left(\left\langle C_{H S}\left(\varpi_{H_{2}}\right) \tau_{i}, \tau_{k}\right\rangle\right)$. Using $\mathrm{R}_{H}=0$ (see Remark1.4 in Section 1.1) yields

$$
\left\langle\nabla_{\tau_{i}}^{H} \nabla_{\tau_{k}}^{H} \tau_{i}, v_{H}\right\rangle=\left\langle\nabla_{\tau_{k}}^{H} \nabla_{\tau_{i}}^{H} \tau_{i}, v_{H}\right\rangle+\left\langle\nabla_{\left[\tau_{i}, \tau_{k}\right]_{H}}^{H} \tau_{i}, v_{H}\right\rangle \quad \forall i, k \in I_{H S} . .
$$

Therefore

$$
\sum_{i \in I_{H S}} A_{i}=\underbrace{-\left\langle v_{H}, \nabla_{\tau_{k}}^{H}\left(\sum_{i \in I_{H S}} \nabla_{\tau_{i}}^{H} \tau_{i}\right)\right\rangle}_{=: A}-\sum_{i \in I_{H S}}\left(\left\langle\nabla_{\left[\tau_{i}, \tau_{k}\right]_{H}}^{H} \tau_{i}, v_{H}\right\rangle+\tau_{i}\left(\left\langle C_{H S}\left(\varpi_{H_{2}}\right) \tau_{i}, \tau_{k}\right\rangle\right)\right) .
$$

We claim that $A=0$ at $p$. Indeed, by hypothesis, $\mathcal{H}_{H}=\left\langle\sum_{i \in I_{H S}} \nabla_{\tau_{i}}^{H} \tau_{i}, v_{H}\right\rangle$ is constant. Hence we get that $\left\langle\sum_{i \in I_{H S}} \nabla_{\tau_{i}}^{H} \tau_{i}, \nabla_{\tau_{k}}^{H} \nu_{H}\right\rangle=0$ at $p$. Furthermore, since at $p \in S$ one has $\left[\tau_{i}, \tau_{k}\right]_{H}=\left\langle C_{H S}\left(\varpi_{H_{2}}\right) \tau_{i}, \tau_{k}\right\rangle v_{H}$ $\forall i, k \in I_{H S}$, it follows that

$$
\begin{aligned}
\sum_{i \in I_{H S}} A_{i} & =\sum_{i \in I_{H S}}\left(\left\langle C_{H S}\left(\varpi_{H_{2}}\right) \tau_{i}, \tau_{k}\right\rangle\left\langle\nabla_{v_{H}^{H}} v_{H}, \tau_{i}\right\rangle-\tau_{i}\left(\left\langle C_{H S}\left(\varpi_{H_{2}}\right) \tau_{i}, \tau_{k}\right\rangle\right)\right) \\
& =-\left(\left\langle\nabla_{v_{H}}^{H} \nu_{H}, C_{H S}\left(\varpi_{H_{2}}\right) \tau_{k}\right\rangle+\sum_{i \in I_{H S}} \tau_{i}\left(\left\langle C_{H S}\left(\varpi_{H_{2}}\right) \tau_{i}, \tau_{k}\right\rangle\right)\right) .
\end{aligned}
$$

Finally, (ii) will follow from the next calculation:

$$
\begin{aligned}
\tau_{i}\left(\left\langle C_{H S}\left(\varpi_{H_{2}}\right) \tau_{i}, \tau_{k}\right\rangle\right) & =\sum_{\alpha \in I_{V}}\left(\tau_{i}\left(\varpi_{\alpha}\right)\left\langle C_{H}^{\alpha} \tau_{i}, \tau_{k}\right\rangle+\varpi_{\alpha}\left(\left\langle C_{H}^{\alpha} \nabla_{\tau_{i}}^{H} \tau_{i}, \tau_{k}\right\rangle+\left\langle C_{H}^{\alpha} \tau_{i}, \nabla_{\tau_{i}}^{H} \tau_{k}\right\rangle\right)\right) \\
& =\sum_{\alpha \in I_{V}}\left(\tau_{i}\left(\varpi_{\alpha}\right)\left\langle C_{H}^{\alpha} \tau_{i}, \tau_{k}\right\rangle+\varpi_{\alpha}\left(-\left\langle\nabla_{\tau_{i}}^{H} \tau_{i}, v_{H}\right\rangle\left\langle C_{H}^{\alpha} \tau_{k}, v_{H}\right\rangle+\left\langle C_{H}^{\alpha} \tau_{i}, v_{H}\right\rangle\left\langle\nabla_{\tau_{i}}^{H} \tau_{k}, v_{H}\right\rangle\right)\right) \\
& =\sum_{\alpha \in I_{V}} \tau_{i}\left(\varpi_{\alpha}\right)\left\langle C_{H}^{\alpha} \tau_{i}, \tau_{k}\right\rangle+\mathcal{H}_{H}\left\langle C_{H}\left(\varpi_{H_{2}}\right) \nu_{H}, \tau_{k}\right\rangle-B_{H}\left(C_{H}\left(\varpi_{H_{2}}\right), \tau_{k}\right) .
\end{aligned}
$$

Using (i) of Lemma 5.2 yields the following "folklore" result:

Proposition 5.3. Let $S \subset \mathbb{G}$ be a $\mathbf{C}^{2}$ hypersurface of constant horizontal mean curvature $\mathcal{H}_{H}$. Then, at each non-characteristic point $p \in S \backslash C_{S}$, we have:

(i) $\left\langle\overrightarrow{\Delta_{H S}} v_{H}, v_{H}\right\rangle=-\left\|B_{H}\right\|_{\mathrm{Gr}}^{2}$;

(ii) $\overrightarrow{\Delta_{H S}} x_{H}=\mathcal{H}_{H} v_{H}$.

Below we shall compute the $H S$-laplacian $\mathcal{L}_{H S}$ of the function $f_{H}:=\left\langle V_{H}, v_{H}\right\rangle$, where $V_{H} \in \mathfrak{X}(H)$ is a constant horizontal left invariant vector field. 
Lemma 5.4. Let $S \subset \mathbb{G}$ be $\mathbf{C}^{2}$ hypersurface of constant horizontal mean curvature $\mathcal{H}_{H}$. Then

$$
-\mathcal{L}_{H S} f_{H}=f_{H}\left\|B_{H}\right\|_{\mathrm{G} r}^{2}+\left\langle\nabla_{v_{H}^{H}}^{H} v_{H}, C_{H S}\left(\varpi_{H_{2}}\right) V_{H S}\right\rangle+\sum_{\alpha \in I_{V}}\left\langle C_{H}^{\alpha} \operatorname{grad}_{H S} \varpi_{\alpha}, V_{H S}\right\rangle+\mathcal{H}_{H}\left\langle C_{H}\left(\varpi_{H_{2}}\right) v_{H}, V_{H S}\right\rangle
$$

at each non-characteristic point $p \in S \backslash C_{S}$.

Proof. Fix a point $p \in S \backslash C_{S}$ and choose a moving frame centered at $p$; see Lemma 3.9 We have

$$
\begin{aligned}
\Delta_{H S} f_{H}= & \sum_{i \in I_{H S}} \tau_{i} \tau_{i}\left(\left\langle V_{H}, v_{H}\right\rangle\right)=\sum_{i \in I_{H S}} \tau_{i}\left(\left\langle V_{H}, \nabla_{\tau_{i}}^{H} \nu_{H}\right\rangle\right)=\sum_{i \in I_{H S}}\left(\left\langle V_{H}, \nabla_{\tau_{i}}^{H} \nabla_{\tau_{i}}^{H} v_{H}\right\rangle\right) \\
= & -\left(f_{H}\left\|B_{H}\right\|_{\mathrm{G} r}^{2}+\left\langle\nabla_{v_{H}}^{H} \nu_{H}, C_{H S}\left(\varpi_{H_{2}}\right) V_{H S}\right\rangle+\sum_{\alpha \in I_{V}}\left\langle C_{H}^{\alpha} \operatorname{grad}_{H S} \varpi_{\alpha}, V_{H S}\right\rangle\right. \\
& \left.+\mathcal{H}_{H}\left\langle C_{H}\left(\varpi_{H_{2}}\right) v_{H}, V_{H S}\right\rangle-B_{H}\left(C_{H}\left(\varpi_{H_{2}}\right) v_{H}, V_{H S}\right)\right),
\end{aligned}
$$

where we have used (i) and (ii) of Lemma 5.2. The thesis follows since

$$
B_{H}\left(C_{H}\left(\varpi_{H_{2}}\right) v_{H}, V_{H S}\right)=-\left\langle C_{H}\left(\varpi_{H_{2}}\right) \nu_{H}, \operatorname{grad}_{H S} f_{H}\right\rangle .
$$

A simple consequence of this lemma, at least from a "formal" point of view, is that, in general, the function $f_{H}$ cannot be an eigenfunction of a linear eigenvalue problem $\mathcal{L}_{H S} \varphi+\lambda \mathcal{B} \varphi=0$, where $\mathcal{B}$ is a given smooth function on $S \backslash C_{S}$. This is a big difference compared with the Euclidean case where, for any constant vector field $V \in \mathbb{R}^{n}$, the function $f=\langle V, v\rangle$ is always a solution to the linear equation $\Delta_{T S} \varphi+\left\|B_{\mathcal{R}}\right\|_{\mathrm{Gr}}^{2} \varphi=0$. Here $\Delta_{T S}$ is the Laplace-Beltrami operator on $S$ and $B_{\mathcal{R}}$ is the 2nd fundamental form of $S$. This equation says that $V$ is a Killing field of any constant mean curvature hypersurface $S \subset \mathbb{R}^{n}$; see [40]. Nevertheless, we have the following:

Lemma 5.5. Let $S \subset \mathbb{G}$ be a $\mathbf{C}^{2}$ hypersurface of constant horizontal mean curvature. Then

$$
-\mathcal{L}_{H S} \varpi_{\alpha}=\varpi_{\alpha} \mathcal{B}_{T S} \quad \forall \alpha \in I_{V}
$$

at each non-characteristic point $p \in S \backslash C_{S}$.

Proof. For the sake of simplicity, we shall assume that $f$ is a NDF; see Definition 3.11, Let $\underline{\tau}$ be an adapted moving frame along $S$. We have $\operatorname{grad}_{H} f=\tau_{1}$ (and hence $\tau_{1}(f)=1$ ) and $\tau_{\alpha} f=\varpi_{\alpha}$ for every $\alpha \in I_{V}$. We stress that $\frac{\partial \varpi_{\alpha}}{\partial \tau_{1}}=X_{\alpha}\left(\frac{\partial f}{\partial \tau_{1}}\right)=X_{\alpha}(1)=0$. Using Lemma 5.1 yields

$$
\begin{aligned}
\Delta_{H S} \varpi_{\alpha} & =\Delta_{H} \varpi_{\alpha}-\left\langle\operatorname{Hess}_{H}\left(\varpi_{\alpha}\right) \tau_{1}, \tau_{1}\right\rangle \\
& =\Delta_{H}\left(\tau_{\alpha} f\right)-\left\langle\operatorname{Hess}_{H}\left(\tau_{\alpha} f\right) \tau_{1}, \tau_{1}\right\rangle \\
& =\tau_{\alpha}\left(\Delta_{H}(f)\right)-\left\langle\nabla_{\tau_{\alpha}}\left(\operatorname{Hess}_{H}(f)\right) \tau_{1}, \tau_{1}\right\rangle \\
& =\varpi_{\alpha} \tau_{1}\left(\Delta_{H}(f)\right)-\left\langle\nabla_{\tau_{\alpha}}\left(\operatorname{Hess}_{H}(f)\right) \tau_{1}, \tau_{1}\right\rangle \\
& =-\varpi_{\alpha} \tau_{1}\left(\mathcal{H}_{H}\right)-\left\langle\nabla_{\tau_{\alpha}}\left(\mathcal{J}_{H} \tau_{1}\right) \tau_{1}, \tau_{1}\right\rangle .
\end{aligned}
$$

Since $\left\langle\left(\mathcal{J}_{H} \tau_{1}\right) \tau_{1}, \tau_{1}\right\rangle=0$, we get that $\left\langle\nabla_{\tau_{\alpha}}\left(\left(\mathcal{J}_{H} \tau_{1}\right) \tau_{1}\right), \tau_{1}\right\rangle=-\left\langle\left(\mathcal{J}_{H} \tau_{1}\right) \tau_{1}, \nabla_{\tau_{\alpha}} \tau_{1}\right\rangle$ and hence

$$
\left\langle\left(\mathcal{J}_{H} \tau_{1}\right) \nabla_{\tau_{\alpha}} \tau_{1}, \tau_{1}\right\rangle+\left\langle\nabla_{\tau_{\alpha}}\left(\mathcal{J}_{H} \tau_{1}\right) \tau_{1}, \tau_{1}\right\rangle=-\left\langle\left(\mathcal{J}_{H} \tau_{1}\right) \tau_{1}, \nabla_{\tau_{\alpha}} \tau_{1}\right\rangle \quad \forall \alpha \in I_{V} .
$$

But since $\left\langle\left(\mathcal{J}_{H} \tau_{1}\right) \nabla_{\tau_{\alpha}} \tau_{1}, \tau_{1}\right\rangle=0$, we obtain

$$
\left\langle\nabla_{\tau_{\alpha}}\left(\mathcal{J}_{H} \tau_{1}\right) \tau_{1}, \tau_{1}\right\rangle=-\left\langle\left(\mathcal{J}_{H} \tau_{1}\right) \tau_{1}, \nabla_{\tau_{\alpha}} \tau_{1}\right\rangle=-\left\langle\nabla_{\tau_{1}}^{H} \tau_{1}, \operatorname{grad}_{H} \varpi_{\alpha}\right\rangle .
$$

By using (i) of Lemma 3.13, it follows that $\nabla_{\tau_{1}}^{H} \tau_{1}=-C_{H}\left(\varpi_{H_{2}}\right) \tau_{1}$ and so, by adding the quantity $\left\langle C_{H}\left(\varpi_{H_{2}}\right) \tau_{1}, \operatorname{grad}_{H S} \varpi_{\alpha}\right\rangle$, we finally get the identity $\mathcal{L}_{H S} \varpi_{\alpha}=-\varpi_{\alpha} \tau_{1}\left(\mathcal{H}_{H}\right)$. The quantity $\tau_{1}\left(\mathcal{H}_{H}\right)$ can 
be obtained by repeating the calculations made in the proof of the 2 nd variation formula. We have

$$
\begin{aligned}
& -\tau_{1}\left(\mathcal{H}_{H}\right) \\
& =\operatorname{div}_{H S}\left(C_{H}\left(\varpi_{H_{2}}\right) \tau_{1}\right)-\left|C_{H}\left(\varpi_{H_{2}}\right) \tau_{1}\right|^{2}+\left\|A_{H}\right\|_{\mathrm{G} r}^{2}-\left\|S_{H}\right\|_{\mathrm{G} r}^{2}+\sum_{j \in I_{H S} \in I_{V}}\left\langle C_{H}^{\alpha} \tau_{1}, \tau_{j}\right\rangle\left(\left\langle C(\varpi) \tau_{\alpha}, \tau_{j}\right\rangle-\tau_{j}\left(\varpi_{\alpha}\right)\right) \\
& =-\left\|A_{H}\right\|_{\mathrm{G} r}^{2}-\left\|S_{H}\right\|_{\mathrm{G} r}^{2}-\sum_{\alpha \in I_{V}}\left\langle\left(2 \operatorname{grad}_{H S}\left(\varpi_{\alpha}\right)-C(\varpi) \tau_{\alpha}^{T S}\right), C^{\alpha} \tau_{1}\right\rangle \\
& =-\mathcal{B}_{T S} .
\end{aligned}
$$

In Section 6.1, just as an exercise, we will reprove this identity for the class of non-vertical hyperplanes

$$
\mathcal{I}_{\alpha^{\prime}}:=\left\{p \equiv \exp \left(\sum_{j=1}^{n} x_{j}\right) \in \mathbb{G}: x_{\alpha^{\prime}}=0\right\},
$$

where $\alpha^{\prime} \in I_{V}$; see Definition 1.11 For the sake of simplicity, this will be done only for 2-step Carnot groups. We recall that these hyperplanes are very different from the vertical ones and, for instance, they turn out to be characteristic at the identity $0 \in \mathbb{G}$. We now state an immediate consequence of the previous lemma. To this aim, let $V \in \mathfrak{X}(\mathbb{G})$ be a constant left invariant vector field.

Corollary 5.6. Let $S \subset \mathbb{G}$ be a $\mathbf{C}^{2}$ hypersurface of constant horizontal mean curvature. Then the function $f_{V}:=\langle V, \varpi\rangle$ satisfies the equation $-\mathcal{L}_{H S} f_{V}=f_{V} \mathcal{B}_{T S}$ at each non-characteristic point of $S$.

\section{Stability of $H$-Minimal hyPERSURFaces}

Definition 6.1 (Stability). Let $\mathbb{G}$ be a k-step Carnot group and let $S \subset \mathbb{G}$ be a $H$-minimal hypersurface of class $\mathbf{C}^{3}$.

$\left(S_{1}\right)$ If $C_{S}=\emptyset$, we say that $S$ is stable if $I I_{S}\left(\sigma_{H}^{n-1}\right) \geq 0$ for every $\mathbf{C}^{3}$-smooth compactly supported variation $\left.\vartheta_{t}:\right]-\epsilon, \epsilon[\times S \longrightarrow \mathbb{G}$.

$\left(S_{2}\right)$ If $C_{S} \neq \emptyset$, we further assume that $\frac{1}{\left|\mathcal{P}_{H} \nu\right|^{2}} \in L^{2}\left(S, \sigma_{\mathcal{R}}^{n-1}\right)$. Then, we say that $S$ is stable if $I I_{S}\left(\sigma_{H}^{n-1}\right) \geq 0$ for every $\mathbf{C}^{3}$-smooth compactly supported variation $\left.\vartheta_{t}:\right]-\epsilon, \epsilon[\times S \longrightarrow \mathbb{G}$ for which there exists $h \in L^{1}\left(\mathcal{U} ; \sigma_{\mathcal{R}}^{n-1}\right)$ such that $\frac{1}{\left|\mathcal{P}_{H_{t}} \nu^{t}\right|} \leq h$ for every $\left.t \in\right]-\epsilon, \epsilon[$.

Remark 6.2. We shall sometimes say that $S$ is strictly stable when the stability inequality is strict. If $C_{S} \neq \emptyset$, but we use only compactly supported variations on $S^{*}:=S \backslash C_{S}$, then $\left(S_{1}\right)$ applies to any non-characteristic domain $\Omega \Subset S^{*}$.

Lemma 6.3. Let $S \subset \mathbb{G}$ be as in Definition 6.1 and let us consider the following linear eigenvalue problem, i.e.

$$
\left\{\begin{aligned}
\mathcal{L}_{H S} \varphi+\lambda \mathcal{B}_{T S} \varphi & =0 & & \text { on } S \\
\varphi & =0 & & \text { on } \partial S .
\end{aligned}\right.
$$

Under the previous assumptions, a sufficient condition for stability of $S$ is that the first (non-trivial) eigenvalue $\lambda_{1}$ of this problem is greater than or equal to 1; see Notation 4.14

Proof. This is an immediate consequence of the horizontal Green formula (6); see Corollary 2.8

Lemma 6.4. Let $S \subset \mathbb{G}$ be a hypersurface of class $\mathbf{C}^{2}$. Let $\Omega \subset S^{*}=S \backslash C_{S}$ be a bounded noncharacteristic domain and let $q \in \mathbf{C}(\Omega)$. If there exists a smooth function $\psi>0$ on $\Omega$ satisfying the equation $\mathcal{L}_{H S} \psi=q \psi$, then

$$
\int_{\Omega}\left(\left|\operatorname{grad}_{H S} \varphi\right|^{2}+q \varphi^{2}\right) \sigma_{H}^{n-1} \geq 0
$$

for all smooth function $\varphi$ compactly supported on $\Omega$. 
This lemma generalizes a well-known result in the Riemannian setting; see [31].

Proof of Lemma 6.4 If $\psi>0$ satisfies $\mathcal{L}_{H S} \psi=q \psi$ on $\Omega$, let us define a new function $\phi:=\log \psi$. By an elementary calculation we see that $\mathcal{L}_{H S} \phi=q-\left|\operatorname{grad}_{H S} \phi\right|^{2}$. More precisely, we have

$$
\begin{aligned}
\mathcal{L}_{H S} \phi & =\operatorname{div}_{H S}\left(\operatorname{grad}_{H S} \phi\right)+\left\langle C_{H}\left(\varpi_{H_{2}}\right) v_{H}, \operatorname{grad}_{H S} \phi\right\rangle \\
& =\operatorname{div}_{H S}\left(\frac{\operatorname{grad}_{H S} \psi}{\psi}\right)+\left\langle C_{H}\left(\varpi_{H_{2}}\right) v_{H}, \frac{\operatorname{grad}_{H S} \psi}{\psi}\right\rangle \\
& =\left(\frac{\Delta_{H S} \psi}{\psi}\left\langle C_{H}\left(\varpi_{H_{2}}\right) v_{H}, \frac{\operatorname{grad}_{H S} \psi}{\psi}\right\rangle\right)-\frac{\left|\operatorname{grad}_{H S} \psi\right|^{2}}{\psi^{2}} \\
& =\frac{\mathcal{L}_{H S} \psi}{\psi}-\left|\operatorname{grad}_{H S} \phi\right|^{2} \\
& =q-\left|\operatorname{grad}_{H S} \phi\right|^{2} .
\end{aligned}
$$

So let $\varphi$ be a smooth function with compact support on $\Omega$. Multiplying by $-\varphi^{2}$ both sides of this equation and integrating by parts, yields

(40) $-\int_{\Omega} \varphi^{2}\left(q-\left|\operatorname{grad}_{H S} \phi\right|^{2}\right) \sigma_{H}^{n-1}=-\int_{\Omega} \varphi^{2} \mathcal{L}_{H S} \phi \sigma_{H}^{n-1}=\int_{\Omega} 2 \varphi\left\langle\operatorname{grad}_{H S} \varphi, \operatorname{grad}_{H S} \phi\right\rangle \sigma_{H}^{n-1}$,

where we have used Corollary 2.8. Note that

$$
2\left|\varphi\left\langle\operatorname{grad}_{H S} \varphi, \operatorname{grad}_{H S} \phi\right\rangle\right| \leq 2|\varphi|\left|\operatorname{grad}_{H S} \varphi \| \operatorname{grad}_{H S} \phi\right| \leq|\varphi|^{2}\left|\operatorname{grad}_{H S} \phi\right|^{2}+\left|\operatorname{grad}_{H S} \varphi\right|^{2} .
$$

Hence, (39) follows inserting (41) into (40) and canceling the terms $\int_{\Omega} \varphi^{2}\left|\operatorname{grad}_{H S} \phi\right|^{2} \sigma_{H}^{n-1}$.

As a consequence of Lemma 5.5 and Lemma 6.4, we infer an interesting condition for stability.

Theorem 6.5. Let $S \subset \mathbb{G}$ be a H-minimal hypersurface of class $\mathbf{C}^{3}$. If there exists $\alpha \in I_{V}$ such that either $\varpi_{\alpha}>0$ or $\varpi_{\alpha}<0$ on $S$, then each non-characteristic domain $\Omega \subset S^{*}$ turns out to be stable.

Proof. By applying Lemma6.4 to the function $\varpi_{\alpha}$ we immediately get the stability inequality

$$
I_{S}\left(W, \sigma_{H}^{n-1}\right) \geq 0
$$

for every non-zero compactly supported variation $\vartheta_{t}$ of $S$.

We have the following reformulations of Theorem 6.5 .

Corollary 6.6. Let $S \subset \mathbb{G}$ be a $H$-minimal hypersurface of class $\mathbf{C}^{3}$. Let $V \in \mathfrak{X}(\mathbb{G})$ be a constant left invariant vector field and set $f_{V}=\langle V, \varpi\rangle$. If either $f_{V}>0$ or $f_{V}<0$, then each non-characteristic domain $\Omega \subset S^{*}$ is stable.

Corollary 6.7. Let $S \subset \mathbb{G}$ be a complete H-minimal hypersurface of class $\mathbf{C}^{3}$. If $S$ is a graph with respect to a given vertical direction, then each non-characteristic domain $\Omega \subset S^{*}$ is stable.

Below we shall study some (more or less simple) examples in order to illustrate some of our results.

6.1. Examples. Our first example, which is that of vertical hyperplanes, is the simplest one and, to the best of our knowledge, the only known in literature outside the Heisenberg group setting. Roughly speaking, vertical hyperplanes are level-sets of linear homogeneous polynomial having (homogeneous) degree 1 , which are ideals of the Lie algebra $\mathfrak{g}$.

We claim that they are (strictly) stable hypersurfaces. This immediately follows from the fact that $\mathcal{B}_{T S}=0$. Hence, for any regular bounded domain $\mathcal{U}$ contained on a vertical hyperplane $\mathcal{I}$, we have $I_{\mathcal{U}}\left(W, \sigma_{H}^{n-1}\right)=\int_{\mathcal{U}}\left|\operatorname{grad}_{H S} w\right|^{2} \sigma_{H}^{n-1} \geq 0$, with equality if, and only if, $w=0$.

Corollary 6.8. Let $\mathbb{G}$ be a $k$-step Carnot group. A vertical hyperplane is a $\mathbf{C}^{\infty}$-smooth non-characteristic strictly stable H-minimal hypersurface. 
Now we analyze a completely different family of hyperplanes. From an intrinsic point of view, they are homogeneous "cones", which turn out to be characteristic at a single point. For the sake of simplicity, we just consider the case of 2-step Carnot groups. We have $\mathfrak{g}=H \oplus V(\operatorname{dim} H=h, \operatorname{dim} V=n-h)$. Let us assume that

$$
X_{i}(x):=\mathrm{e}_{i}+\frac{1}{2} \sum_{\alpha \in I_{V}}\left\langle C_{H}^{\alpha} \mathrm{e}_{i}, x_{H}\right\rangle \mathrm{e}_{\alpha}, \quad X_{\alpha}=\mathrm{e}_{\alpha}
$$

for every $i \in I_{H}=\{1, \ldots, h\}$ and every $\alpha \in I_{V}=\{h+1, \ldots, n\}$, where $\mathrm{e}_{j}=(0, \ldots, \underbrace{1}_{j-\text { thplace }}, \ldots 0), j=1 \ldots, n$, is the $j$-th vector of the canonical basis of $\mathbb{R}^{n} \cong \mathfrak{g}$ and $x_{H} \equiv\left(x_{1}, \ldots, x_{h}\right)$ is the horizontal position vector. As usual, we identify vector fields and differential operators.

Fix $\alpha^{\prime} \in I_{V}$ and consider the non-vertical hyperplane $\mathcal{I}_{\alpha^{\prime}}:=\left\{x=\exp \left(\sum_{j} x_{j}\right) \in \mathbb{G}: x_{\alpha^{\prime}}=0\right\}$. We have $\operatorname{grad}_{H} x_{\alpha^{\prime}}=-\frac{1}{2} C_{H}^{\alpha^{\prime}} x_{H}$ and so $v_{H}=\frac{-C_{H}^{\alpha^{\prime}} x_{H}}{\left|C_{H}^{\alpha^{\prime}} x_{H}\right|}$. Moreover, $\varpi_{\beta}=0$ for all $\beta \neq \alpha^{\prime}$ and $\varpi_{\alpha^{\prime}}=\frac{2}{\left|C_{H}^{\alpha^{\prime}} x_{H}\right|}$. Since

$$
\operatorname{div}_{H}\left(C_{H}^{\alpha^{\prime}} x_{H}\right)=\sum_{j \in I_{H}}\left\langle\nabla_{X_{j}} C_{H}^{\alpha^{\prime}} x_{H}, X_{j}\right\rangle=\sum_{j \in I_{H}}\left\langle C_{H}^{\alpha^{\prime}} X_{j}, X_{j}\right\rangle=0
$$

and

$$
\left\langle\operatorname{grad}_{H}\left(\frac{1}{\left|C_{H}^{\alpha^{\prime}} x_{H}\right|}\right), C_{H}^{\alpha^{\prime}} x_{H}\right\rangle=-\left\langle\frac{\operatorname{grad}_{H}\left|C_{H}^{\alpha^{\prime}} x_{H}\right|}{\left|C_{H}^{\alpha^{\prime}} x_{H}\right|^{2}}, C_{H}^{\alpha^{\prime}} x_{H}\right\rangle=\left\langle\frac{C_{H}^{\alpha^{\prime}} v_{H}}{\left|C_{H}^{\alpha^{\prime}} x_{H}\right|}, v_{H}\right\rangle=0,
$$

it follows that $\mathcal{H}_{H}=-\operatorname{div}_{H} v_{H}=0$, i.e. $\mathcal{I}_{\alpha^{\prime}}$ is $H$-minimal. The above calculation also shows that $\operatorname{grad}_{H}\left(\left|C_{H}^{\alpha^{\prime}} x_{H}\right|\right)=C_{H}^{\alpha^{\prime}} v_{H}$. Furthermore, we easily get that

$$
-\mathcal{J}_{H} v_{H}=\frac{C_{H}^{\alpha^{\prime}}+v_{H} \otimes C_{H}^{\alpha^{\prime}} v_{H}}{\left|C_{H}^{\alpha^{\prime}} x_{H}\right|}
$$

which, in turn, implies

$$
B_{H}\left(\tau_{i}, \tau_{j}\right)=\left\langle\frac{C_{H S}^{\alpha^{\prime}}}{\left|C_{H}^{\alpha^{\prime}} x_{H}\right|} \tau_{i}, \tau_{j}\right\rangle=A_{H}\left(\tau_{i}, \tau_{j}\right) \quad \forall i, j \in I_{H S} .
$$

Therefore $S_{H}=\mathbf{0}_{H}$ (i.e. the 0-matrix on $H$ ) and $\left\|B_{H}\right\|_{\mathrm{G} r}^{2}=\left\|A_{H}\right\|_{\mathrm{G} r}^{2}=\frac{\varpi_{\alpha^{\prime}}^{2}\left\|C_{H S}^{\alpha^{\prime}}\right\|_{\mathrm{G} r}^{2}}{4}$. It remains to compute the quantity $\Upsilon:=-\sum_{\alpha \in I_{V}}\left\langle\left(2 \operatorname{grad}_{H S}\left(\varpi_{\alpha}\right)-C(\varpi) \tau_{\alpha}^{T S}\right), C^{\alpha} \tau_{1}\right\rangle$; see formula (37). Since we are in a 2-step group, we have

$$
\Upsilon=-\sum_{\alpha \in I_{V}}\left\langle\left(2 \operatorname{grad}_{H S}\left(\varpi_{\alpha}\right)+\varpi_{\alpha} C(\varpi) \tau_{1}\right), C^{\alpha} \tau_{1}\right\rangle .
$$

From the previous calculations, it follows that $\Upsilon=0$ and so $\mathcal{B}_{T S}=\left\|A_{H}\right\|_{\mathrm{G} r}^{2}$. In other words, we have

$$
I_{\mathcal{U}}\left(W, \sigma_{H}^{n-1}\right)=\int_{\mathcal{U}}\left(\left|\operatorname{grad}_{H S} w\right|^{2}-w^{2}\left\|A_{H}\right\|_{\mathrm{G} r}^{2}\right) \sigma_{H}^{n-1}=\int_{\mathcal{U}}\left(\left|\operatorname{grad}_{H S} w\right|^{2}-w^{2} \frac{\varpi_{\alpha^{\prime}}^{2}\left\|C_{H S}^{\alpha^{\prime}}\right\|_{\mathrm{G} r}^{2}}{4}\right) \sigma_{H}^{n-1}
$$

for any non-characteristic bounded domain $\mathcal{U} \subset \mathcal{I}_{\alpha^{\prime}}(\equiv S)$, where $\sigma_{H}^{n-1}\left\llcorner\mathcal{I}_{\alpha^{\prime}}=\frac{\left|C_{H}^{\alpha^{\prime}} x_{H}\right|}{2} d \mathcal{L}_{\mathrm{Eu}}^{n-1}\left\llcorner\mathcal{I}_{\alpha^{\prime}}\right.\right.$ and

$$
d \mathcal{L}_{\mathrm{Eu}}^{n-1}=d x_{1} \wedge d x_{2} \wedge \ldots \wedge \ldots \wedge \widehat{d x_{\alpha^{\prime}}} \wedge \ldots \wedge d x_{n}
$$

It goes without saying that the previous formula holds true near the characteristic set only under the assumptions made in Corollary 4.13. In particular, we have to check that $\int_{\mathcal{U}} \frac{1}{\mid \mathcal{P}_{H} v^{4}} \sigma_{\mathcal{R}}^{n-1}<+\infty$, which is clearly equivalent to the next condition:

$$
\int_{\mathcal{U}} \frac{1}{\left|C_{H}^{\alpha^{\prime}} x_{H}\right|^{4}} d \mathcal{L}_{\mathrm{Eu}}^{n-1}\left\llcorner\mathcal{I}_{\alpha^{\prime}}<+\infty\right.
$$

Two remarks are in order: 
- a necessary condition for the validity of (42) is that the dimension of $H$ is $\geq 5$, i.e.

$$
h=\operatorname{dim} H \geq 5
$$

- in the Heisenberg group $\mathbb{H}^{n}$, the previous analysis reduces to the case of the horizontal hyperplane $\left\{p=\exp (z, t) \in \mathbb{H}^{n}: t=0\right\}$ and, in this case, (43) is also sufficient for (42) to hold.

Now let us compute

$$
\begin{aligned}
\Delta_{H S}\left(\frac{1}{\left|C_{H}^{\alpha^{\prime}} x_{H}\right|}\right) & =-\operatorname{div}_{H S}\left(\frac{C_{H}^{\alpha^{\prime}} v_{H}}{\left|C_{H}^{\alpha^{\prime}} x_{H}\right|^{2}}\right) \\
& =\frac{2\left|C_{H}^{\alpha^{\prime}} v_{H}\right|^{2}}{\left|C_{H}^{\alpha^{\prime}} x_{H}\right|^{3}}-\frac{\operatorname{div}_{H S}\left(C_{H}^{\alpha^{\prime}} v_{H}\right)}{\left|C_{H}^{\alpha^{\prime}} x_{H}\right|^{2}} \\
& =\frac{2\left|C_{H}^{\alpha^{\prime}} v_{H}\right|^{2}}{\left|C_{H}^{\alpha^{\prime}} x_{H}\right|^{3}}+\frac{\sum_{j, k \in I_{H S}}\left\langle\nabla_{\tau_{j}}^{H} \tau_{1}, \tau_{k}\right\rangle\left\langle C_{H}^{\alpha^{\prime}} \tau_{j}, \tau_{k}\right\rangle}{\left|C_{H}^{\alpha^{\prime}} x_{H}\right|^{2}} \\
& =\frac{2\left|C_{H}^{\alpha^{\prime}} v_{H}\right|^{2}-\left\|C_{H S}^{\alpha^{\prime}}\right\|_{\mathrm{G} r}^{2}}{\left|C_{H}^{\alpha^{\prime}} x_{H}\right|^{3}} .
\end{aligned}
$$

From this computation and the very definition of $\mathcal{L}_{H S}$, it follows that

$$
\mathcal{L}_{H S}\left(\frac{1}{\left|C_{H}^{\alpha^{\prime}} x_{H}\right|}\right)=\Delta_{H S}\left(\frac{1}{\left|C_{H}^{\alpha^{\prime}} x_{H}\right|}\right)+\left\langle C_{H}\left(\varpi_{H_{2}}\right) v_{H}, \operatorname{grad}_{H S}\left(\frac{1}{\left|C_{H}^{\alpha^{\prime} x_{H}}\right|}\right)\right\rangle=-\frac{\left\|C_{H S}^{\alpha^{\prime}}\right\|_{\mathrm{G} r}^{2}}{\left|C_{H}^{\alpha^{\prime}} x_{H}\right|^{3}},
$$

which is equivalent to the equation $\mathcal{L}_{H S} \varpi_{\alpha^{\prime}}=-\varpi_{\alpha^{\prime}}\left\|A_{H}\right\|_{G r}^{2}$, as predicated by Lemma5.5.

The previous discussion is summarized in the following:

Corollary 6.9. Let $\mathbb{G}$ be a 2-step Carnot group and let $\mathcal{I}_{\alpha^{\prime}}$ be a horizontal hyperplane passing through $0 \in \mathbb{G}$. Then $\mathcal{I}_{\alpha^{\prime}}$ is a $\mathbf{C}^{\infty}$-smooth $H$-minimal hypersurface. The only characteristic point of $\mathcal{I}_{\alpha^{\prime}}$ is the identity $0 \in \mathbb{G}$. Furthermore, any bounded domain $\mathcal{U} \Subset \mathcal{I}_{\alpha^{\prime}} \backslash\{0\}$ turns out to be strictly stable.

6.2. An example in the Heisenberg group $\mathbb{H}^{n}$. For the notation used in this section we refer the reader to Example 1.5 and Example 1.8. We recall that any point $p \in \mathbb{H}^{n}$ is identified with $(z, t) \in \mathbb{R}^{2 n+1}$, where $z=\left(x_{1}, y_{1}, x_{2}, y_{2}, \ldots, x_{n}, y_{n}\right)$. We use the following further notation:

$\bar{v}^{1,0}:=\left(v_{1}, 0, v_{2}, 0, \ldots, v_{n}, 0\right) \in \mathbb{R}^{2 n}, \quad \bar{v}^{0,1}:=\left(0, v_{1}, 0, v_{2}, 0, \ldots, 0, v_{n}\right) \in \mathbb{R}^{2 n} \quad \forall v=\left(v_{1}, v_{2}, \ldots, v_{n}\right) \in \mathbb{R}^{n}$.

Using this notation yields $z=\bar{x}^{1,0}+\bar{y}^{0,1} \in \mathbb{R}^{2 n}$, where $x=\left(x_{1}, x_{2}, \ldots, x_{n}\right) \in \mathbb{R}^{n}$ and $y=\left(y_{1}, y_{2}, \ldots, y_{n}\right) \in \mathbb{R}^{n}$. In the sequel, we shall study the following hyperbolic paraboloid:

$$
S:=\left\{p \equiv(z, t) \in \mathbb{H}^{n}: t=\frac{\left\|\bar{x}^{1,0}\right\|_{\mathbb{R}^{n}}^{2}-\left\|\bar{y}^{1,0}\right\|_{\mathbb{R}^{n}}^{2}}{4}\right\},
$$

First, note that $\operatorname{grad}_{H} t=\frac{z^{\circ}}{2}$, where $z^{\circ}:=-C_{H}^{2 n+1} z$. Furthermore, a simple calculation shows that $\operatorname{grad}_{H}\left(\frac{\left\|\bar{x}^{1,0}\right\|_{\mathbb{R}^{n}}^{2}-\left\|\bar{y}^{1,0}\right\|_{\mathbb{R}^{n}}^{2}}{4}\right)=\frac{1}{2}\left(\bar{x}^{1,0}-\bar{y}^{0,1}\right)$ and hence $v_{H}=\frac{-\bar{v}^{1,0}+\bar{v}^{0,1}}{\left|-\bar{v}^{1,0}+\bar{v}^{0,1}\right|}$, where we have set $v=x+y \in \mathbb{R}^{n}$. Therefore

$$
v_{H}=\frac{\sqrt{2}}{2}\left(\frac{-\bar{v}^{1,0}+\bar{v}^{0,1}}{\sqrt{\rho^{2}+2\langle x, y\rangle_{\mathbb{R}^{n}}}}\right), \quad v_{H}^{\circ}=-\frac{\sqrt{2}}{2}\left(\frac{\bar{v}^{1,0}+\bar{v}^{0,1}}{\sqrt{\rho^{2}+2\langle x, y\rangle_{\mathbb{R}^{n}}}}\right),
$$

where $\sqrt{\rho^{2}+2\langle x, y\rangle_{\mathbb{R}^{n}}}=\|x+y\|_{\mathbb{R}^{n}}$ and $\rho:=\sqrt{\|x\|_{\mathbb{R}^{n}}^{2}+\|y\|_{\mathbb{R}^{n}}^{2}}$. Clearly, the characteristic set $C_{S}$ of $S$ is the set of all points $p \equiv(z, t) \in S$ such that $\overline{x+y}^{1,0}=\overline{x+y}^{0,1}=0 \in \mathbb{R}^{2 n}$. Hence $p \equiv(z, t) \in C_{S}$ if, and only if $x_{i}=-y_{i}$ for every $i=1, \ldots, n$. Since $X_{i}\left(\frac{1}{\|x+y\|_{\mathbb{R}^{n}}}\right)=Y_{i}\left(\frac{1}{\|x+y\|_{\mathbb{R}^{n}}}\right)$, we easily get that $d i v_{H} v_{H}=0$, i.e. $S$ is $H$-minimal. 
We have $\varpi=\frac{\sqrt{2}}{\|x+y\|_{\mathbb{R}^{n}}}$ and $\frac{\partial \varpi}{\partial v_{H}^{\circ}}=\frac{2}{\|x+y\|_{\mathbb{R}^{n}}^{2}}$. In order to calculate the horizontal 2nd fundamental form $B_{H}$ (and some of its invariants) we need the horizontal Jacobian matrix $\mathcal{J}_{H} v_{H}=:\left[a_{i j}\right]_{i, j \in I_{H}}$ of the $H$-normal $v_{H}$. For the sake of simplicity, we treat the case $n=2$, which corresponds to the 2nd Heisenberg group. The general case is completely analogous. We have

- $a:=a_{11}=a_{12}=-\frac{\sqrt{2}}{2}\left(\frac{\|x+y\|_{\mathbb{R}^{2}}^{2}-\left(x_{1}+y_{1}\right)^{2}}{\|x+y\|_{\mathbb{R}^{2}}^{3}}\right), \quad b:=a_{13}=a_{14}=-\frac{\sqrt{2}}{2}\left(\frac{-\left(x_{1}+y_{1}\right)\left(x_{2}+y_{2}\right)}{\|x+y\|_{\mathbb{R}^{2}}^{3}}\right) ;$

- $a_{2 j}=-a_{1 j}$ for every $j=1, \ldots, 4$;

- $a_{31}=a_{32}=-\frac{\sqrt{2}}{2}\left(\frac{-\left(x_{1}+y_{1}\right)\left(x_{2}+y_{2}\right)}{\|x+y\|_{\mathbb{R}^{2}}^{3}}\right), \quad c:=a_{33}=a_{34}=-\frac{\sqrt{2}}{2}\left(\frac{\|x+y\|_{\mathbb{R}^{2}}^{2}-\left(x_{2}+y_{2}\right)^{2}}{\|x+y\|_{\mathbb{R}^{2}}^{3}}\right)$;

- $a_{4 j}=-a_{3 j}$ for every $j=1, \ldots, 4$.

Equivalently, $\mathcal{J}_{H} v_{H}=\left[\begin{array}{cccc}a & a & b & b \\ -a & -a & -b & -b \\ b & b & c & c \\ -b & -b & -c & -c\end{array}\right]$. It follows that $v_{H} \in \operatorname{Ker} \mathcal{J}_{H} v_{H}$ and hence $B_{H}=-\mathcal{J}_{H} v_{H}$. By definition, we have $S_{H}=-\left(\frac{\mathcal{J}_{H} v_{H}+\left(\mathcal{J}_{H} v_{H}\right)^{\mathrm{Tr}}}{2}\right)=-\left[\begin{array}{cccc}a & 0 & b & 0 \\ 0 & -a & 0 & -b \\ b & 0 & c & 0 \\ 0 & -b & 0 & -c\end{array}\right]$. So if $n=2$, we have

$$
\left\|B_{H}\right\|_{\mathrm{G} r}^{2}=4\left(a^{2}+2 b^{2}+c^{2}\right)=\frac{2}{\|x+y\|_{\mathbb{R}^{n}}^{2}}, \quad\left\|S_{H}\right\|_{\mathrm{G} r}^{2}=2\left(a^{2}+2 b^{2}+c^{2}\right)=\frac{1}{\|x+y\|_{\mathbb{R}^{n}}^{2}}=\left\|A_{H}\right\|_{\mathrm{G} r}^{2} .
$$

In the general case, an analogous calculation gives $\left\|B_{H}\right\|_{\mathrm{G} r}^{2}=\frac{2(n-1)}{\|x+y\|_{\mathbb{R}^{n}}^{2}}$ and $\left\|S_{H}\right\|_{\mathrm{G} r}^{2}=\left\|A_{H}\right\|_{\mathrm{G} r}^{2}=\frac{n-1}{\|x+y\|_{\mathbb{R}^{n}}^{2}}$. Therefore, using (38) yields

$$
\begin{aligned}
\mathcal{B}_{T S} & =\left\|S_{H}\right\|_{G r}^{2}-\left(2 \frac{\partial \varpi}{\partial v_{H}^{\circ}}-\frac{n+1}{2} \varpi^{2}\right) \\
& =\frac{n-1}{\|x+y\|_{\mathbb{R}^{n}}^{2}}-\left(\frac{4}{\|x+y\|_{\mathbb{R}^{n}}^{2}}-\frac{n+1}{\|x+y\|_{\mathbb{R}^{n}}^{2}}\right) \\
& =\frac{2(n-2)}{\|x+y\|_{\mathbb{R}^{n}}^{2}} .
\end{aligned}
$$

Remark 6.10 ( $B_{T S}$ can be negative!). If $n=1$, then $B_{T S}<0$ for any non-characteristic domain $\mathcal{U} \subset S$.

The previous calculation implies that

$$
I_{\mathcal{U}}\left(W, \sigma_{H}^{2 n}\right)=\int_{\mathcal{U}}\left(\left|\operatorname{grad}_{H S} w\right|^{2}-w^{2} \frac{2(n-2)}{\|x+y\|_{\mathbb{R}^{n}}^{2}}\right) \sigma_{H}^{2 n}
$$

for any non-characteristic bounded domain $\mathcal{U} \subset S$, where $\sigma_{H}^{2 n}=\frac{\|x+y\|_{\mathbb{R}^{n}}}{\sqrt{2}} d z$ and we have set

$$
d z=d x_{1} \wedge d y_{1} \wedge \ldots \wedge d x_{n} \wedge d y_{n} .
$$

Remark 6.11 (Failure of $\int_{\mathcal{U}} \frac{1}{\left|\mathcal{P}_{H} v\right|^{4}} \sigma_{\mathcal{R}}^{n-1}<+\infty$ for characteristic domains). In order to apply the previous 2 nd variation formula for a characteristic domain $\mathcal{U} \subset S$, we need (at least) to check that

$$
\int_{\mathcal{U}} \frac{1}{\|x+y\|_{\mathbb{R}^{n}}^{4}} d \mathcal{L}_{\mathrm{Eu}}^{2 n}<+\infty
$$

However, in general, this condition fails to hold if $C_{\mathcal{U}} \neq \emptyset$. 


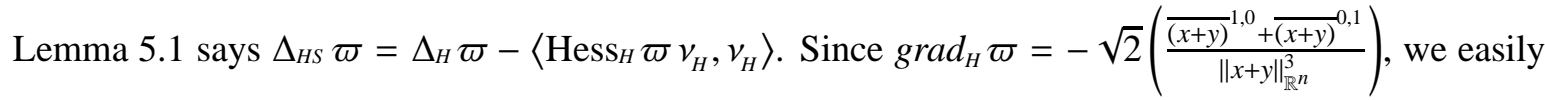
get that $\Delta_{H} \varpi=-\varpi \frac{(2 n-3)}{\|x+y\|_{\mathbb{R}^{n}}^{2}}$. Furthermore

$$
\left.\left\langle\operatorname{Hess}_{H} \varpi v_{H}, v_{H}\right\rangle=\left\langle\begin{array}{ccccc}
1 & 1 & 0 & 0 & \ldots \\
1 & 1 & 0 & 0 & \ldots \\
0 & 0 & 1 & 1 & \ldots \\
0 & 0 & 1 & 1 & \ldots \\
\ldots & \ldots & \ldots & \ldots & \ldots
\end{array}\right] v_{H}, v_{H}\right\rangle=-\frac{\varpi}{\|x+y\|_{\mathbb{R}^{n}}^{2}} .
$$

All together, we have shown that

$$
\mathcal{L}_{H S} \varpi=\Delta_{H S} \varpi-\varpi \frac{\partial \varpi}{\partial v_{H}^{\circ}}=-\varpi \frac{2(n-2)}{\|x+y\|_{\mathbb{R}^{n}}^{2}},
$$

which illustrates the content of Lemma 5.5 .

Corollary 6.12. Let $S=\left\{p \equiv(z, t) \in \mathbb{H}^{n}: t=\frac{\left\|\bar{x}^{1,0}\right\|_{\mathbb{R}^{n}}^{2}-\left\|\bar{y}^{1,0}\right\|_{\mathbb{R}^{n}}^{2}}{4}\right\}$, where $z:=\bar{x}^{1,0}+\bar{y}^{0,1} \in \mathbb{R}^{2 n}$. Then $S$ turns out to be a $\mathbf{C}^{\infty}$-smooth H-minimal hypersurface. Furthermore, one has

$$
C_{S}=\left\{p=\exp (z, t) \in S: x_{i}=-y_{i}, i=1, \ldots, n\right\} .
$$

Finally, any bounded domain $\mathcal{U} \Subset S \backslash C_{S}$ is strictly stable.

\section{REFERENCES}

[1] W.K. Allard, On the First Variation of a Varifold, Ann. Math. (2) 95, 417-491 (1972).

[2] - On the first variation of a varifold: Boundary behavior, Ann. Math. (2) 101, 418-446 (1975).

[3] L. Ambrosio, N. Fusco, D. Pallara, "Functions of bounded variation and free discontinuity problems ", Oxford Mathematical Monographs. The Clarendon Press, Oxford University Press, New York (2000).

[4] L. Ambrosio, S. Rigot, Optimal mass transportation in the Heisenberg group, J. Funct. Anal. 208, no. 2, 261-301 (2004).

[5] L. Ambrosio, F. Serra Cassano, D. Vittone, Intrinsic regular hypersurfaces in Heisenberg groups, J. Geom. Anal. 16, no. 2, 187-232 (2006).

[6] L. Ambrosio, B. Kleiner, E. Le Donne, Rectifiability of sets of finite perimeter in Carnot groups: existence of a tangent hyperplane, J. Geom. Anal. 19 , no. 3, 509-540 (2009)

[7] G. Anzellotti, Pairings between measures and bounded functions and compensated compactness, Ann. Mat. Pura Appl., IV. Ser. 135, 293-318 (1983)

[8] N. Arcozzi, F. Ferrari \& F. Montefalcone, CC-distance and metric normal of smooth hypersurfaces in sub-Riemannian Carnot groups, preprint available at ArXiv.

[9] Z.M. Balogh, J.T. Tyson, B. Warhurst, Sub-Riemannian vs. Euclidean dimension comparison and fractal geometry on Carnot groups, Adv. Math. 220, no. 2, 560-619 (2009).

[10] J.L Barbosa, M. Do Carmo, Stability of Hypersurfaces with constant mean curvature, Math. Z. 185, 339-353 (1984).

[11] J.L Barbosa, M. Do Carmo, J.Eschenburg, Stability of Hypersurfaces with constant mean curvature in Riemannian Manifolds, Math. Z. 197, 123-138 (1988).

[12] Z.M. Balogh, C. Pintea, H. Rohner, Size of tangencies to non-involutive distributions, to appear in Indiana University Mathematics Journal

[13] V. Barone Adesi, F. Serra Cassano, D.Vittone, The Bernstein problem for intrinsic graphs in Heisenberg groups and calibrations, Calc. Var. Partial Differential Equations 30, no. 1, 17-49 (2007).

[14] E. Bombieri, “An introduction to minimal currents and parametric variational problems”, Math. Rep. 2, no. 3, 285-384 (1985).

[15] I. Chavel, "Riemannian Geometry: a modern introduction”, Cambridge University Press, 1994.

[16] - Isoperimetric Inequalities, Cambridge University Press, 2001.

[17] J.J Cheng, J.F. Hwang, A. Malchiodi, P. Yang, Minimal surfaces in pseudohermitian geometry, Ann. Sc. Norm. Sup. Pisa Cl. Sci., 5, IV, 129-179 (2005).

[18] - A Codazzi-like equation and the singular set for C1 smooth surfaces in the Heisenberg group, J. Reine Angew. Math., to appear

[19] J.J Cheng, J.F. Hwang, P. YAng, Regularity of $C^{1}$ smooth surfaces with prescribed p-mean curvature in the Heisenberg group, Math. Ann. 344, No. 1, 1-35 (2009).

[20] G.Q Chen, M. Torres, W.P. Ziemer, Gauss-Green theorem for weakly differentiable vector fields, sets of finite perimeter, and balance laws, Commun. Pure Appl. Math. 62, No. 2, 242-304 (2009).

[21] S.S. Chern, Minimal submanifolds in a Riemannian manifold, Mimeographed lecture notes, University of Kansas (1968).

[22] L. Capogna, G. Citti, M. Manfredini, Smoothness of Lipschitz minimal intrinsic graphs in Heisenberg groups $H^{n}, n>1$, Indiana University Mathematics Journal (2008).

[23] L. Capogna, D. Danielli, N. Garofalo, The geometric Sobolev embedding for vector fields and the isoperimetric inequality, Comm. Anal. Geom. 12 (1994).

[24] D. Danielli, N. Garofalo, D.M. Nhieu, Minimal surfaces, surfaces of constant mean curvature and isoperimetry in Carnot groups, Preprint (2001).

[25] - Sub-Riemannian Calculus on Hypersurfaces in Carnot groups, Adv. Math. 215, no. 1, 292-378 (2007).

[26] Integrability of the sub-Riemannian mean curvature of surfaces in the Heisenberg group, Proceedings of the A.M.S., Volume 140, Number 3 , March 2012, Pages 811821

[27] E. De Giorgi, Frontiere orientate di misura minima, Seminario di Matematica della Scuola Normale Superiore di Pisa, 196061. Editrice Tecnico Scientifica, Pisa, 1961.

[28] M. Do Carmo, Hypersurfaces of constant mean curvature, Proc. 3rd Int. Symp., Peiscola/Spain 1988, Lect. Notes Math. 1410, 128-144 (1989).

[29] H. Federer, “Geometric Measure Theory”, Springer Verlag, 1969.

[30] H. Federer, W.H. Fleming, Normal and integral currents, Ann. of Math. (2) 72, 458-520 (1960).

[31] D. Fisher-Colbrie, R. Schoen, The structure of complete stable minimal surfaces in 3-manifolds of non-negative scalar curvature, Communications of Pure and Applied Mathematics, vol. XXXIII, 119-211 (1980).

[32] B. Franchi, R. Serapioni, F.S. Cassano, Rectifiability and Perimeter in the Heisenberg Group, Math. Ann., 321, 479-531 (2001).

[33] - Regular hypersurfaces, intrinsic perimeter and implicit function theorem in Carnot groups, Comm. Anal. Geom., 11, no. 5, 909-944 (2003).

[34] On the structure of finite perimeter sets in step 2 Carnot groups, J. Geom. Anal., 13, no. 3, 421-466 (2003)

[35] - Regular submanifolds, graphs and area formula in Heisenberg groups., Adv. Math. 211, no. 1, 152-203 (2007) . 
[36] N. Garofalo, D.M. Nhieu, Isoperimetric and Sobolev inequalities for Carnot-Carathéodory spaces and the existence of minimal surfaces, Comm. Pure Appl. Math., 49 (1996) 1081-1144.

[37] N. Garofalo, S. Pauls, The Bernstein problem in the Heisenberg group, arXiv:math.DG/0209065.

[38] E. Giusti, "Minimal surfaces and functions of bounded variation”, Monographs in Mathematics, Vol. 80, Birkhuser, Boston-Basel-Stuttgart (1984).

[39] P.A Griffiths, Exterior Differential Systems and the Calculus of Variations, Progress in Mathematics, Birkhauser 1982.

[40] M. Gromov,Carnot-Carathéodory spaces seen from within, in "Subriemannian Geometry", Progress in Mathematics, 144. ed. by A. Bellaiche and J. Risler, Birkhauser Verlag, Basel (1996).

[41] R. Hermann, The 2nd variation for Minimal Submanifolds, Journal of Mathematics and Mechanics, vol. 16, n. 5 (1966).

[42] R.H. HLadKy, S.D. Pauls, Constant mean curvature surfaces in sub-Riemannian geometry, J. Differential Geom. 79, no. 1, 111-139 (2008)

[43] R.H Hladky, S.D. Pauls, Variation of Perimeter Measure in sub-Riemannian geometry, ArXiv:math/0702237

[44] A. Hurtado, M. Ritoré, C. Rosales, The classification of complete stable area-stationary surfaces in the Heisenberg group, Adv. Math. 224, No. 2, 561-600 (2010).

[45] J. Jost, X.Li Jost, Calculus of Variations, Cambridge Studies in Advanced Mathematics, 64, Cambridge University Press, Cambridge, 1998.

[46] S.G. Krantz, H.R.. Parks, "The geometry of domains in space”, Birkhuser Advanced Texts. Boston, MA: Birkhuser. x, 308 p. (1999).

[47] H.B. Lawson, "Lectures on Minimal Submanifolds", Publish or Perish, Inc., Berkeley, 1980

[48] J.M. LeE, Introduction to Smooth Manifolds, Springer Verlag, 2003.

[49] J.H. Michael, L.M. Simon, Sobolev and Mean-value Inequalities on Generalized Submanifolds of $\mathbb{R}^{n}$, Comm. on Pure and Applied Math., Vol. XXVI, 361-379 (1973).

[50] V. Magnani, “Elements of Geometric Measure Theory on sub-Riemannian groups", PHD Thesis, Scuola Normale Superiore di Pisa, (2002).

[51] Characteristic points, Rectifiability and Perimeter measure on stratified groups, J. Eur. Math. Soc. (JEMS), 8, no. 5, 585-609 (2006).

[52] V. Magnani, D. Vittone, An intrinsic measure for submanifolds in stratified groups, J. Reine Angew. Math. 619, 203-232 (2008).

[53] V. MAzJa, "Sobolev Spaces”, Springer-Verlag (1985).

[54] F. Montefalcone, "Some Remarks in Differential and Integral Geometry of Carnot Groups" Tesi di Dottorato- Università di Bologna- Aprile 2004

[55] - Some relations among volume, intrinsic perimeter and one-dimensional restrictions of BV functions in Carnot groups Annali della Scuola Normale Superiore di Pisa, Classe di Scienze, 5, IV, Fasc.1, 79-128 (2005).

[56] - Hypersurfaces and variational formulae in sub-Riemannian Carnot groups, Journal de Mathématiques Pures et Appliquées, 87, 453-494 (2007).

[57] - Isoperimetric and Sobolev inequalities on hypersurfaces in sub-Riemannian Carnot groups, preprint available at ArXiv.

[58] R. Montgomery, "A Tour of Subriemannian Geometries, Their Geodesics and Applications", AMS, Math. Surveys and Monographs, 91, 2002.

[59] R. Monti, F. Serra Cassano, Surface measures in Carnot-Carathéodory spaces, Calc. Var. and Pde, 13, $339-376$ (2001).

[60] F. Morgan, "Geometric measure theory. A beginner's guide”, Illustrated by James F. Bredt. 4th ed. (English) Amsterdam: Elsevier/Academic Press. viii, 249 p. (2009).

[61] R. Osserman, "A Survey of Minimal Surfaces”, Van Nostrand Reinhold Mathematical Studies. 25. New York etc.: Van Nostrand Reinhold Company, 159 p. (1969).

[62] P. PAnsu, “Geometrie du Group d'Heisenberg”, These pour le titre de Docteur, 3ème cycle, Universite Paris VII, 1982.

[63] - Submanifolds and differential forms in Carnot manifolds, after M. Gromov et M. Rumin. (2005).

[64] S.D. Pauls, Minimal surfaces in the Heisenberg group, Geom. Dedicata, 104, 201-231 (2004).

[65] W. Pfeffer, "Derivation and integration", Cambridge Tracts in Mathematics. 140. Cambridge: Cambridge University Press. xvi, 266 p. (2001).

[66] M. RitoRÉ, Examples of area-minimizing surfaces in the sub-Riemannian Heisenberg group $\mathbb{H}^{1}$ with low regularity, Calc. Var. Partial Differ. Equ. 34, No. 2 , 179-192 (2009).

[67] M. Ritoré, C. Rosales, Area stationary surfaces in the Heisenberg group $\mathbb{H}^{1}$, Adv. Math. 219, No. 2, 633-671 (2008)

[68] T. Schick, Analysis on $\delta$-manifolds of bounded geometry, Hodge-de Rham isomorphism and $L^{2}$-index theorem,Berichte aus der Mathematik. Aachen: Shaker Verlag. ii, 111 p. (1996).

[69] M. Shubin, Essential self-adjointness for semi-bounded magnetic Schrdinger operators on non-compact manifolds, J. Funct. Anal. 186, No.1, 92-116 (2001).

[70] L.M. Simon, Lectures on geometric measure theory, Proceedings of the Centre for Mathematical Analysis, Australian National University, Vol. 3 (1983).

[71] J. Simons, Minimal varieties in Riemannian manifolds, Ann. of Math. 88, 62-105 (1968).

[72] M. SPIVAK, "Differential Geometry”, Volume IV, Publisch or Perish, 1964.

[73] E.M. SteIn, "Harmonic Analysis”, Princeton University Press, 1993.

[74] R.S. Strichartz, Sub-Riemannian geometry, J. Diff. Geom., 24 (1986) 221-263. Corrections: J. Diff. Geom., 30, 595-596 (1989).

[75] M.E. TAYLOR, "Measure Theory and Integration", Graduate Studies in Mathematics, Vol. 76, A.M.S. (2006).

Francescopaolo Montefalcone:

Dipartimento di Matematica

Università degli Studi di Padova,

Via Trieste, 63, 35121 Padova (Italy)

E-mail address: montefal@math.unipd.it 